\title{
A radio census of the massive stellar cluster Westerlund $1^{\star}$
}

\author{
H. Andrews ${ }^{1}$, D. Fenech ${ }^{2}$, R. K. Prinja ${ }^{1}$, J. S. Clark ${ }^{3}$, and L. Hindson ${ }^{4}$ \\ 1 University College London, Gower St, Bloomsbury, London WC1E 6BT, UK \\ e-mail: holly.andrews.16@ucl.ac.uk \\ 2 Cavendish Laboratory, JJ Thomson Avenue, Cambridge CB3 0HE, UK \\ 3 Open University, Walton Hall, Kents Hill, Milton Keynes MK7 6AA, UK \\ ${ }^{4}$ University of Hertfordshire, Hatfield, Hertfordshire AL10 9AB, UK
}

Received 5 July 2019 / Accepted 4 September 2019

\begin{abstract}
Context. Massive stars and their stellar winds are important for a number of feedback processes. The mass lost in the stellar wind can help determine the end-point of the star as a neutron star (NS) or a black hole (BH). However, the impact of mass loss on the post-main sequence evolutionary stage of massive stars is not well understood. Westerlund 1 is an ideal astrophysical laboratory in which to study massive stars and their winds in great detail over a large range of different evolutionary phases.

Aims. We aim to study the radio emission from Westerlund 1, in order to measure radio fluxes from the population of massive stars, and determine mass-loss rates and spectral indices where possible.

Methods. Observations were carried out in 2015 and 2016 with the Australia Telescope Compact Array (ATCA) at 5.5 and $9 \mathrm{GHz}$ using multiple configurations, with maximum baselines ranging from $750 \mathrm{~m}$ to $6 \mathrm{~km}$.

Results. Thirty stars are detected in the radio from the fully concatenated dataset, ten of which are Wolf-Rayet stars (WRs) (predominantly late type WN stars), five yellow hypergiants (YHGs), four red supergiants (RSGs), one luminous blue variable (LBV), the $\mathrm{sgB}[\mathrm{e}]$ star W9, and several OB supergiants. New source detections in the radio are found for five WR stars, and five OB supergiants. These detections lead to evidence for three new OB supergiant binary candidates, which is inferred from derived spectral index limits. Conclusions. Spectral indices and index limits were determined for massive stars in Westerlund 1. For cluster members found to have partially optically thick emission, mass-loss rates were calculated. Under the approximation of a thermally emitting stellar wind and a steady mass-loss rate, clumping ratios were then estimated for eight WRs. Diffuse radio emission was detected throughout the cluster. Detections of knots of radio emission with no known stellar counterparts indicate the highly clumped structure of this intra-cluster medium, likely shaped by a dense cluster wind.
\end{abstract}

Key words. stars: massive - stars: mass-loss - stars: winds, outflows - supergiants - stars: Wolf-Rayet - binaries: general

\section{Introduction}

Massive stars and the outflows formed from their stellar winds are responsible for a number of important feedback processes, of chemical and mechanical origin, which include their immediate surroundings as well as further afield. Massive stars are predominantly found to form in massive stellar clusters or associations. Therefore, it is important to understand not only the stars themselves and their individual evolution, but also the impact on and from their environment, in order to quantify all the physics involved. This includes the chemical and mechanical feedback from individual stars to their circumstellar environment, as well as larger scale feedback processes, such as the production of cosmic rays or galactic superwinds. Understanding the cluster environments can also help us to understand how outflows from these massive clusters may trigger or inhibit star formation in nearby regions.

These massive stars are also progenitors of some of the most exotic endpoints in astrophysics, ending their lives as either a neutron star (NS), or directly collapsing to a black hole (BH), while many massive stars also experience a corresponding supernovae $(\mathrm{SNe})$ explosion. This is of particular importance

* A copy of the reduced images is available at the CDS via anonymous ftp to cdsarc.u-strasbg.fr $(130.79 .128 .5)$ or via http: //cdsarc.u-strasbg.fr/viz-bin/cat/J/A+A/632/A38 when considering the recent discovery of gravitational waves, which occur due to the mergers of these important astrophysical objects; the progenitors of these mergers are believed to be of very large masses themselves (Abbott et al. 2016). These final endpoints are characterised primarily by the initial mass of the star, and the mass lost through its stellar lifetime. The characterisation of the SNe itself can also be found to be influenced by the surrounding environment. A better understanding of the cluster environments in which many of these massive stars live and die can help us to understand the origins of the geometries and any associated asymmetries of $\mathrm{SNe}$ remnants observed today.

Despite the clear motivations behind understanding this field of astrophysics, the evolutionary pathways of these massive stars are not well understood. Even the relative phases of stars beyond the main sequence, where the most massive stars are able to become hydrogen stripped Wolf-Rayet stars (WRs), are not well understood. The range of evolutionary stages experienced by massive stars have direct consequences on their final endpoints.

Massive stars are impacted by factors such as mass loss, rotation speeds, and the possible effect of magnetism, as well as binarity. Quantifying the mass loss through stellar winds can help determine the possible evolutionary pathways for different initial stellar masses, helping to constrain and determine possible progenitors for each type of final stellar endpoint. 
Mass-loss rates are not always consistent between those assumed in evolutionary codes and those from observations, especially for the case of early-type $\mathrm{O}$ and $\mathrm{B}$ stars where discrepancies of up to a factor of ten have been recorded (Puls et al. 2006; Fullerton et al. 2006). One of the ways in which this is impacted is the presence of clumping in the wind, which has a direct impact on the final value of mass loss determined in the wind; the necessary inclusion of a clumping factor in calculations quenches the observed mass loss rates (Prinja et al. 2010; Prinja \& Massa 2013; Sundqvist et al. 2011; Surlan et al. 2012).

Another factor that significantly impacts stellar evolution is binarity. Many WR stars have been determined to be located in binary systems. Almost $70 \%$ of massive stars are believed to reside in binary systems (Sana et al. 2012, 2013), and interactions between the primary and secondary stars, especially for those with close separations, could cause significant levels of mass transfer, affecting the evolution of both stellar components. There are several diagnostic factors by which you can determine the presence of binarity for a stellar system, from radial velocity variations to the presence of hard X-rays. Another way to discover a binary candidate is by measuring a negative spectral index for the star in the radio regime. This indicates the presence of non-thermal emission, which may be attributed to colliding stellar winds (Blomme 2010).

Binarity is also of importance in terms of providing a possible source, via the colliding winds of binaries, as a source for cosmic rays. Single massive stars, especially stars with dense winds, such as WRs, are also a potential source for generating cosmic rays via shocks between the stellar winds and their circumstellar environments (Cesarsky \& Montmerle 1983). The generation of cosmic rays in the stellar winds of massive stars are believed to be possible in the locations of open clusters in particular, where the regions of interstellar material and a possible strong radiation field can help to sustain cosmic rays produced by stellar sources, either via their winds or from the resultant $\mathrm{SNe}$ (Bednarek et al. 2014).

Westerlund $1(\mathrm{Wd} 1)$ provides a unique astrophysical laboratory in order to investigate the stellar evolution of massive stars at a multitude of evolutionary stages, with hundreds of $\mathrm{O}$ and $\mathrm{B}$ stars as well as the largest coeval population of yellow hypergiants (YHGs) in our galaxy. Westerlund 1 is the most massive stellar cluster in the Milky Way, discovered in 1961 by Bengt Westerlund (Westerlund 1961). It has a total mass estimate of $\sim 10^{5} M_{\odot}$ (Clark et al. 2005). It has a current age estimate of $5 \mathrm{Myr}$ and a distance estimate of $5 \mathrm{kpc}$. Recent estimates from Gaia data release two (DR2) have suggested a smaller distance of $\sim 3 \mathrm{kpc}$ may be more appropriate (Aghakhanloo et al. 2019), but other discussions of the cluster distance in light of Gaia data have suggested significant limitations in the use of Gaia to determine Wd1's distance, due to the extended structure of some of the cluster's brighter members (Clark et al. 2019a). For this paper, the estimate of $5 \mathrm{kpc}$ is used.

Westerlund 1 has been previously observed across most of the electromagnetic spectrum, with observations carried out in the infrared (IR), optical, millimetre, and X-ray (Clark et al. 2005, 2008; Negueruela et al. 2010; Crowther et al. 2006; Bonanos 2007; Damineli et al. 2016; Muno et al. 2006). It has been determined to be a source of highly energetic cosmic rays from the observations of the products of cosmic-rays (CRs), TeV $\gamma$ rays, observed from the cluster (Abramowski et al. 2012; Aharonian et al. 2019). It has also previously been observed in the radio with the use of the Australia Telescope Compact Array (ATCA) (Clark et al. 1998; Dougherty et al. 2010, henceforth D10), and most recently, in the millimetre with the Atacama Large
Table 1. Summary of observations taken by ATCA.

\begin{tabular}{|c|c|c|c|}
\hline $\begin{array}{l}\text { Array } \\
\text { config. }\end{array}$ & $\begin{array}{l}\text { Observing } \\
\text { dates }\end{array}$ & $\begin{array}{l}\text { Obs. duration } \\
\text { (h) }\end{array}$ & $\begin{array}{l}\text { Beam } \\
\text { size }\end{array}$ \\
\hline $\begin{array}{l}6 \mathrm{~A} \\
(6 \mathrm{~km})\end{array}$ & $\begin{array}{l}27-29 \\
\text { October } 2015\end{array}$ & 31.77 & $\begin{array}{l}1.71^{\prime \prime} \times 0.99^{\prime \prime} \\
\left(0.790^{\circ}\right)\end{array}$ \\
\hline $\begin{array}{l}1.5 \mathrm{~A} \\
(1.5 \mathrm{~km})\end{array}$ & $\begin{array}{l}25-27 \\
\text { November } 2015\end{array}$ & 16.45 & $\begin{array}{l}3.59^{\prime \prime} \times 1.99^{\prime \prime} \\
\left(-7.23^{\circ}\right)\end{array}$ \\
\hline $\begin{array}{l}1.5 \mathrm{~B} \\
(1.5 \mathrm{~km})\end{array}$ & 3 June 2016 & 15.76 & $\begin{array}{l}4.40^{\prime \prime} \times 2.41^{\prime \prime} \\
\left(-2.73^{\circ}\right)\end{array}$ \\
\hline $\begin{array}{l}750 \mathrm{C} \\
(750 \mathrm{~m})\end{array}$ & $\begin{array}{l}14-15 \\
\text { December } 2015\end{array}$ & 28.37 & $\begin{array}{l}6.98^{\prime \prime} \times 4.44^{\prime \prime} \\
\left(6.28^{\circ}\right)\end{array}$ \\
\hline
\end{tabular}

Notes. In the first column, the maximum baseline for each configuration is given in parentheses. In the last column, the position angle for the beam size is given in the parentheses.

Millimeter Array (ALMA) (Fenech et al. 2018, henceforth F18). The observations discussed in this paper provide a direct followon from the prior radio observations, with improved sensitivity and resolution.

Section 2 details the observations, as well as the data reduction. Section 3 describes the data analysis then carried out in order to determine fluxes and spatial extents. Section 4 introduces the result of the diffuse radio emission detected throughout the cluster. In Sect. 5 we introduce the new radio source detections found, separated into stellar sources, uncatalogued sources found in the previous mm-observations, and further previously uncatalogued radio sources determined (with no optical or mm counterpart). Section 6 goes into detail about the results of the stellar sources, considering the WR population, the cool supergiant and hypergiant population, and other stellar sources, including W9, the luminous blue variable (LBV) W243 and the O and B supergiants. The extended emission of the cluster and its possible origin is discussed in Sect. 7. A summary of our conclusions and possible future avenues for investigation is then provided in Sect. 8.

\section{Observations and data reduction}

Observations were made with the use of ATCA over four different observing periods from October 2015 to June 2016. ATCA contains six radio dishes with a diameter of $22 \mathrm{~m}$ each. Data were taken in four different configurations, with maximum baselines set at $750 \mathrm{~m}(750 \mathrm{C}), 1.5 \mathrm{~km}(1.5 \mathrm{~A}, 1.5 \mathrm{~B})$, and $6 \mathrm{~km}(6 \mathrm{~A})$, with details listed in Table 1. Two of these configurations were set at a largest baseline of $1.5 \mathrm{~km}$, due to a repetition of observations carried out to account for bad weather on the first set of $1.5 \mathrm{~km}$ observations. The data were collected over two spectral windows, with central frequencies $5.5 \mathrm{GHz}$, and $9 \mathrm{GHz}$, and a bandwidth of $2 \mathrm{GHz}$ for each band. Each spectral window contained 2048 channels, with bandwidths of $1 \mathrm{MHz}$. Integration time on source per pointing was $\sim 16 \mathrm{~h}$. The calibrator J1636-4101 was used as a phase calibrator for the $6 \mathrm{~km}$ configuration and 1600-48 was used as a phase calibrator for all other configurations. The source 1934-638 was observed as a bandpass calibrator for all configurations and was also used for flux amplitude calibration. A secondary calibrator, 0823-500, was also observed.

Data reduction and calibration was carried out with the use of MIRIAD (Sault et al. 1995), with additional flagging of data carried out in astronomical image processing software (AIPS) with the use of scripted e-MERLIN RFI mitigation pipeline for 


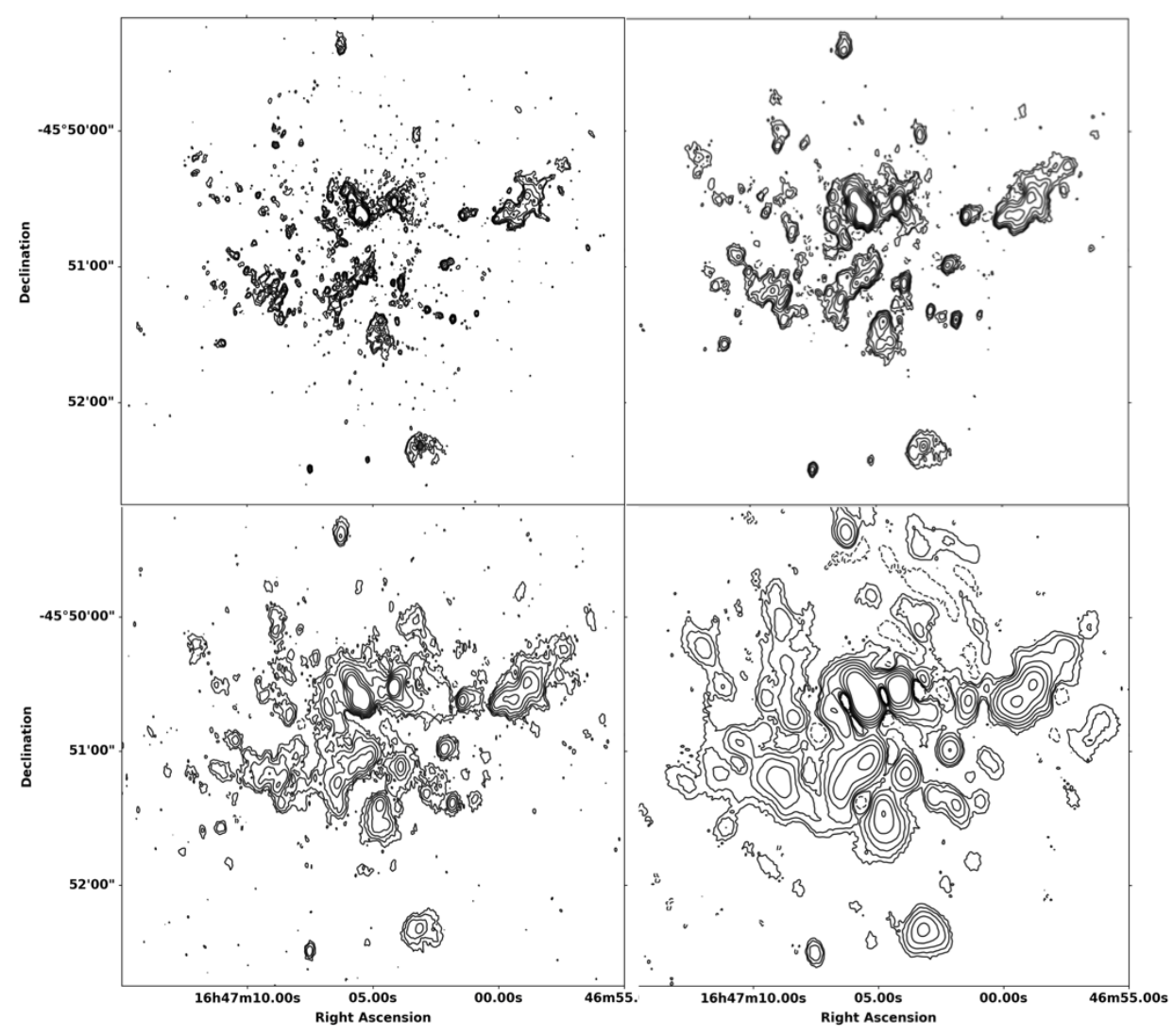

Fig. 1. ATCA contour plots (using nonprimary beam corrected images) combining both spectral windows and separated into four configurations, 6A (top left), 1.5A (top right), 1.5B (bottom left), and 750C (bottom right). Contour levels are set at $-3,3,6,9$, $12,24,48$, and $192 \times \sigma$, with $\sigma=0.02,0.03$, 0.06 , and $0.06 \mathrm{mJy}$ for $6 \mathrm{~A}, 1.5 \mathrm{~A}, 1.5 \mathrm{~B}$, and $750 \mathrm{C}$ respectively. interferometry $\left(\right.$ SERPent ${ }^{1}$ ) on the $9 \mathrm{GHz}$ data (Peck \& Fenech 2013). This involved the flagging of radio frequency interference (RFI) and any erroneous data, as well as calibrating for the bandpass, phase, flux density, and any polarisation leakage, following standard procedures as laid out in the miriad user guide (Sault \& Killeen 2004). After calibration, data were concatenated in several forms, discussed in detail below, with a run-through of both phase and amplitude self-calibration then additionally carried out on these final datasets. This involved the use of CASA (McMullin et al. 2007), where the functions GAINCAL and APPLYCAL were carried out alongside the use of the deconvolution imaging tool TCLEAN, cleaned in the multiscale multi-frequency synthesis mode, with Briggs weighting applied (with the robust parameter set to zero). The psf mode was set to "clark". The resolution was set to be equivalent to the smallest primary beam size present in each dataset. For the fully concatenated dataset, this beam size was taken from the $6 \mathrm{~km}$ observations, $1.71^{\prime \prime} \times 0.99^{\prime \prime}$ (position angle $0.790^{\circ}$ ), as calculated automatically in CASA, and the cell size was set to $0.3 \times 0.3$ arcsec. The multiple deconvolution scales applied were at the size of the beam, as well as additional scales set at 5, 10, $12,15,18,25$, and 47 pixels, in order to consider the varying resolutions when concatenating datasets from different configurations and spectral windows.

This led to a final set of fits files containing fully calibrated and cleaned images. For data analysis, PBCOR (primary beam correction) was applied to mitigate the effects of attenuation from the primary beam for sources further from the pointing centre.

Several datasets were considered for the analysis of the radio observations. The definitive fluxes of radio sources from the cluster are taken from the fully integrated dataset containing

\footnotetext{
1 https://github.com/daniellefenech/SERPent
}

all configurations and all spectral windows observed by ATCA, hereby referred to as the dataset FULLCONCAT. Fluxes were also considered for comparative use from datasets split into the $5.5 \mathrm{GHz}$ and $9 \mathrm{GHz}$, combined over all array configurations, FULL5 and FULL9. These datasets are used for considering the mass-loss rates from each spectral window for thermally emitting sources, and the FULL9 is used to compare flux values to the prior radio observations taken of Wd1 by D10.

Additional datasets generated from the ATCA observations are tapered datasets of both the 5.5 and $9 \mathrm{GHz}$ observations, TAPER 5 and TAPER9. The datasets of tapered visibilities were created in order to compare datasets at different wavelength regimes more accurately, by comparing emission detected only from common ranges of $u-v$ visibilities for both the radio and the millimetre ALMA observations (F18). These datasets were used for the calculation of spectral indices, as well as mass-loss rates, and is discussed in more detail in Sects. 3.5 and 6.1.1. The corresponding mass-loss rates were then used to calculate clumping gradients, as discussed in Sect. 6.1.2. The tapered millimetre observations are referred to as TAPERALMA, in order to distinguish from the results presented in F18, which contains the full $u-v$ range from the ALMA observations.

\section{Analysis}

After data reduction was completed, analysis was carried out on the datasets using SEAC ${ }^{2}$, a source extraction software tool (Peck 2014; Morford et al. 2017). This tool involves the use of PARSELTONGUE, a way to use a python interface with the software AIPS. The data was converted for use from fits files into

\footnotetext{
2 https://github.com/daniellefenech/SEAC
} 


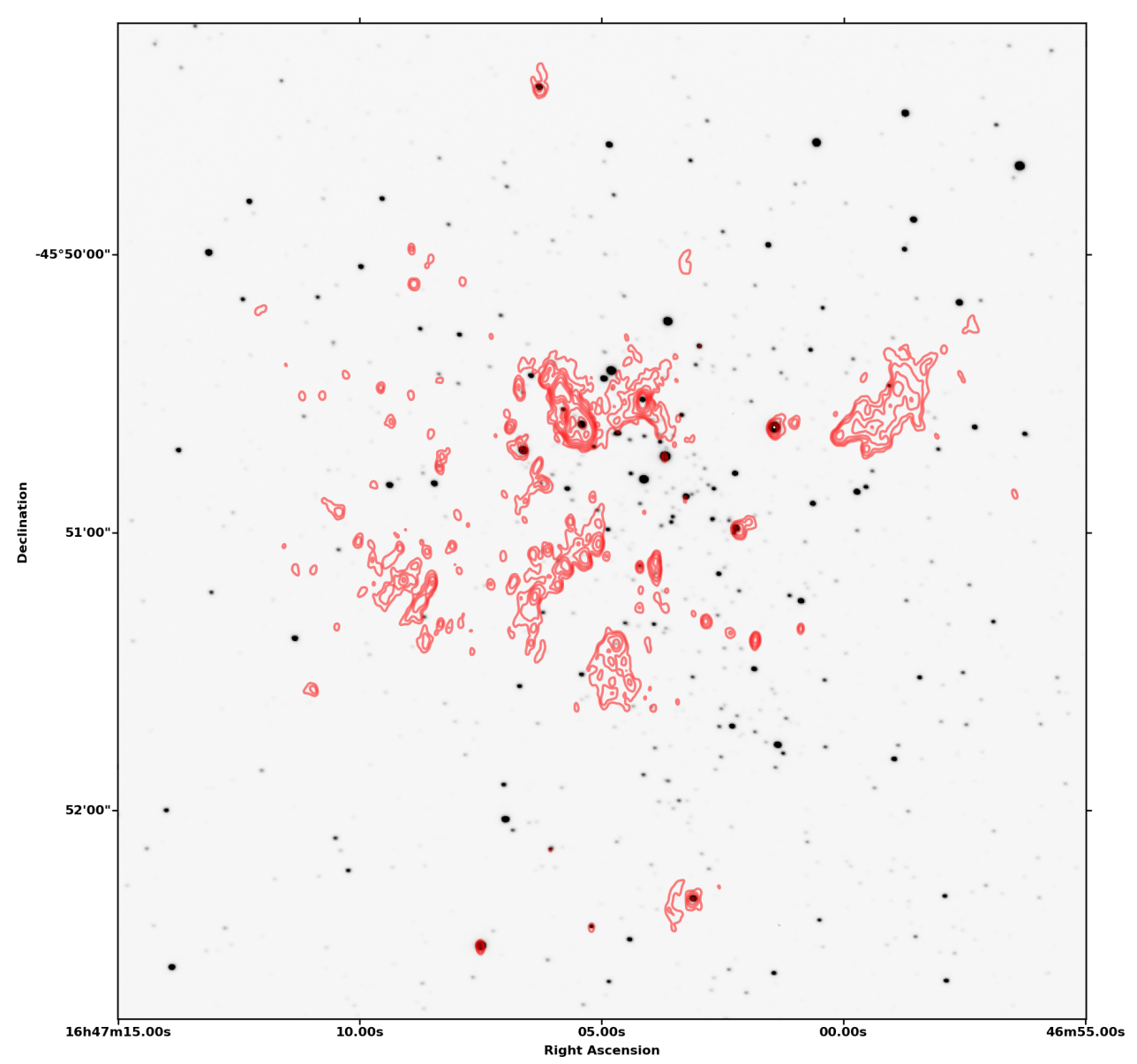

Fig. 2. ATCA contours (in red) from FULLCONCAT dataset (using nonprimary beam corrected image) overlaid on FORS $R$-band image. FORS $R$-band image has a limiting magnitude of 17.5. Contour levels are set at $-3,3$, $6,9,12,24,48,96$, and $192 \sigma$, with $\sigma$ set at $0.015 \mathrm{mJy}$. Contour levels are adjusted in comparison to the contours shown in Fig. 1, in order to better show identification of radio emission with the relevant optical sources. the AIPS environment. A consideration of the possible offset of sources to previous datasets was determined following the procedure of previous radio and millimetre wavelength measurements (D10; F18). The ATCA datasets were run through the AIPS task HGEOM, where the images were aligned to the same geometry as the FORS and ALMA dataset. The data was then run through JMFIT to check any possible offset for the most radio luminous source W9, and only a small offset of the peak position was found. Figure 2 shows the aligned dataset (without primarybeam correction) overlaid as contours on a FORS camera $R$-band image $(655 \mathrm{~nm})(\mathrm{D} 10)$, where contours are scaled by the overall rms uncertainty, $\sigma$, taken to be $0.015 \mathrm{mJy} \mathrm{beam}^{-1}$.

The SEAC software utilises a floodfill algorithm. This algorithm selects initial pixels as possible sources which have values above a specified "seed" threshold. An "island" is then generated by appending adjacent pixels to an array containing just the seed pixel with a peak value initially, until the flux values of these surrounding pixels no longer reach above a specified "flood" threshold. These were set at $5 \sigma$ for the seed threshold and $3 \sigma$ for the flood threshold. The value of $\sigma$ is the rms value of the local spatial region. Local rms values are determined from a generated noise map, created from dividing the full image into a grid with a user-specified number of cells. The rms level is then calculated within each individual cell, and the corresponding cell gives the value of the local noise level, $\sigma$, for each source. This allows for changes in the background radio emission to be considered, especially with regards to possible effects from extended emission of the particularly radio luminous objects W9 and W26, which otherwise could have affected the rms of the total image.

\subsection{Selection of source fluxes}

With the use of SEAC, fluxes were determined from each dataset, for each source. The definitive source fluxes were determined from the detections made in the FULLCONCAT dataset. Many of the sources detected are surrounded by significant levels of radio emission, as discussed further in Sect. 4. Due to this, a measurement of the core components of the sources was carried out using an additional segmentation tool within SEAC. This involved the implementation of the Watershed algorithm (Digabel \& Lantuejoul 1978). SEAC allows the use of two different options in applying this algorithm, either involving a Gaussian filter approach, or using a noise elevation map. Both versions of the segmentation tool were applied to the datasets when trying to pick out accurate core and extended components of the sources, leading to three source output lists for each dataset.

The two possible segmentation algorithms lead to different representations of source emission. Figure 4 demonstrates how applying the two segmentation approaches have clearly different results, shown here for the source WR B, with a non-segmented image taken from the TAPER 9 dataset, shown in Fig. 4b. The NOISE segmentation uses two user-defined thresholds to select the strength of the flux that defines a core component. The "bottom" threshold defines the background noise level to dismiss, and the "top" threshold defines the level of emission above which peaks can be detected. This results in the selection of core components of emission embedded within a larger general area of extended emission, as shown by the brighter shading of the two core components detected in Fig. 4c.

The GAUSs segmentation picks out local maxima of peaks of radio emission, and then applies a Gaussian filter. These 


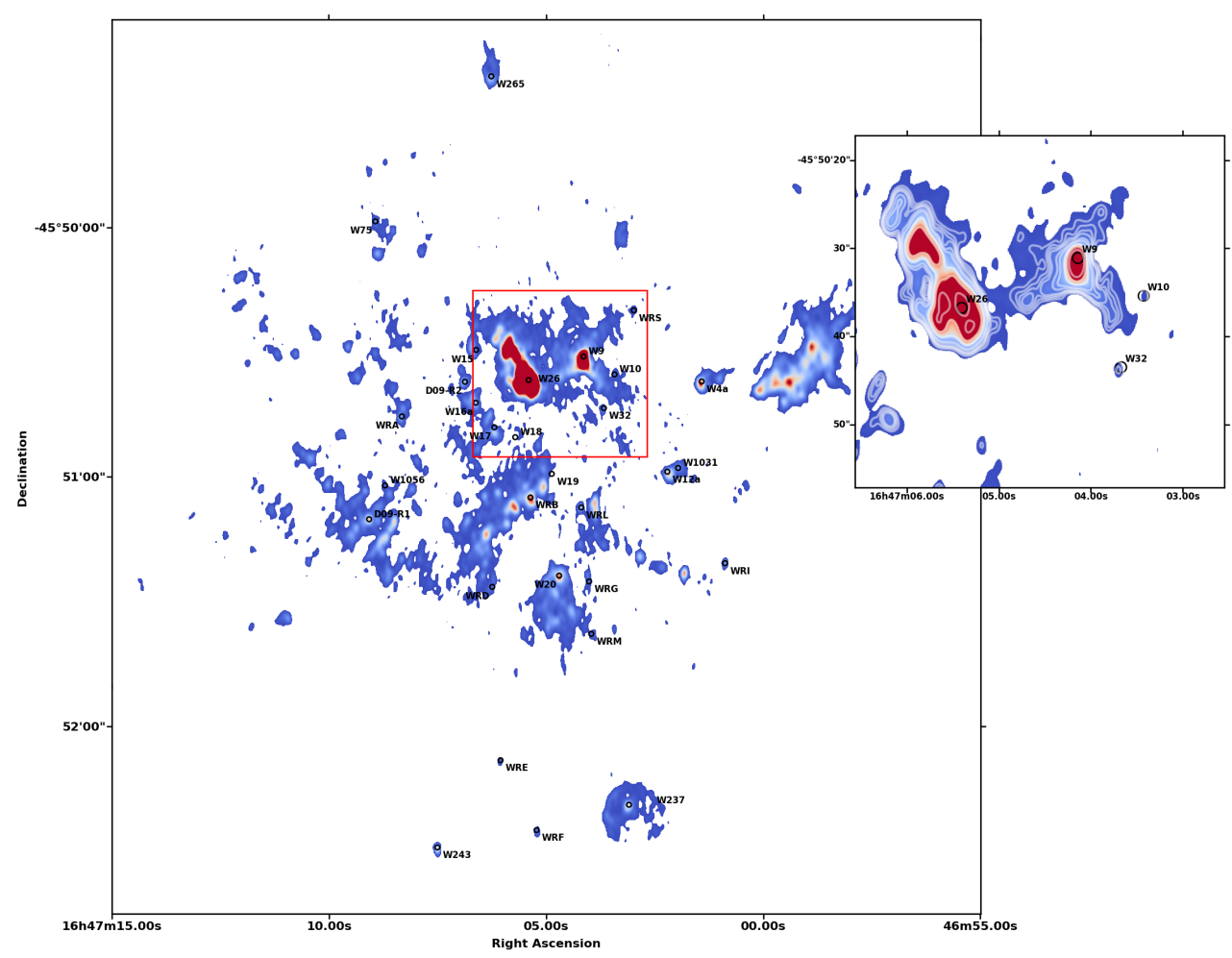

Fig. 3. ATCA colour-scale image (from non-primary beam corrected image) of FULLCONCAT dataset with detected known stellar sources labelled. The colour-scale is set from 0.6 to $2.0 \mathrm{mJy}^{\text {beam }}{ }^{-1}$. The subplot is a zoomed-in image of the central region, with an adjusted colour-scale of $0.15-5.0 \mathrm{mJy} \mathrm{beam}^{-1}$, and overlaid contours with levels of $-3,3,6,8,10$, $12,18,24,48,96,192 \times 45 \mu \mathrm{Jy}^{\text {beam }}{ }^{-1}$. Stellar sources have been identified by comparing the list of known sources from previous observations (D10; F18; Clark et al. 2005, 2019b) to the SEAC detections from the FULLCONCAT dataset.
Gaussians are then smoothed by a user-defined level. This leads to the segmentation of large areas of diffuse emission into smaller groups, helping to segregate out sources which may be effected by crowding, as is seen clearly in Fig. 4d. This method leads to the inclusion of a larger proportion of extended emission than when determining the core flux for a source from the NOISE segmentation. A higher level of smoothing will lead to a smaller number of final segments considered, and will attribute larger levels of surrounding extended emission to the source.

Segmentation was carried out when considering the definitive fluxes of sources taken from FULLCONCAT, as well as when measuring the fluxes to compare the radio observations to the millimetre observations, taken from TAPER5 and TAPER9. It allowed for the core components of sources to be detected, and allowed for further detection of sources embedded in larger regions of continuous diffuse emission surrounding several of the known stellar sources. Segmentation was not carried out for the TAPERALMA, as source structure in ALMA was considered sufficiently compact for all sources, as previously seen in the analysis of the full dataset (F18).

\subsection{Confirmation of ATCA detected sources}

The definitive flux density values were determined from the FULLCONCAT dataset. This is the dataset with the lowest resultant noise level, and the largest integrated flux surrounding each of the sources, so gave the best chance of determining the most complete number of source detections. The decision of final flux density values took into consideration the size of the emission area associated with the source, picked out by SEAC - this required a thorough visual inspection of the SEAC results, for all segmentation options, on each dataset. After source identification was carried out and flux density values were confirmed, further analysis could be applied to the results to calculate physical quantities associated with the sources, including spatial sizes, spectral indices, and mass-loss values.
Errors on the flux densities are given from the combination of the error found by the calculation of integrated flux density in SEAC and the error given for the flux amplitude calibrator 1934-638 (ATNF 2019, online calibrator database). Calibrator errors were listed as $0.1 \%$ at $5.5 \mathrm{GHz}$, and $0.2 \%$ at $9 \mathrm{GHz}$. These two values were then combined in quadrature to give the error from amplitude calibration for the FULLCONCAT dataset. The combined amplitude calibrator error was then combined in quadrature with the SEAC flux error to give final errors for the flux densities. Errors for the fluxes given from the TAPERALMA measurements used a combination in quadrature of the errors found from the flux determination in SEAC and a $5 \%$ error for the absolute amplitude calibration error, following the prescription in F18. However, due the presence of the diffuse emission, it is unclear whether the boundary measured is the true boundary between emission truly related to nearby stellar sources, and emission from the diffuse background. Errors quoted in this paper are therefore potentially underestimates of the true uncertainty on these values, and should be considered as conservative.

\subsection{Spatial extent}

The determination of spatial sizes follows a similar prescription to the method carried out in F18. Source sizes were measured from the FULLCONCAT dataset. Measurements of the spatial extents were made using JMFIT in AIPS to determine a Gaussian fit for the source. Peak flux values and pixel locations for sources as calculated from SEAC were used as central positions from which to measure the source structure and provide a Gaussian model fit to the data. A threshold of surrounding pixels above $3 \sigma$ of the local rms was applied. Convolved and deconvolved source sizes were then determined from this. For sources where there were large levels of surrounding extended emission, the size of the core component was given an initial estimate manually, instead of using the $3 \sigma$ threshold. There were 


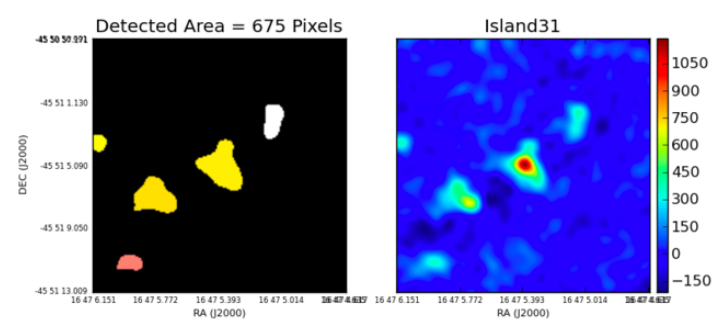

(a)

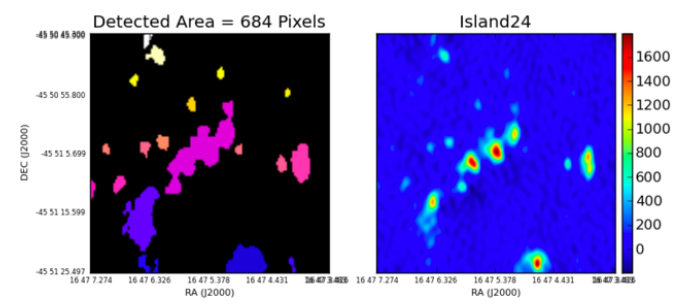

(b)

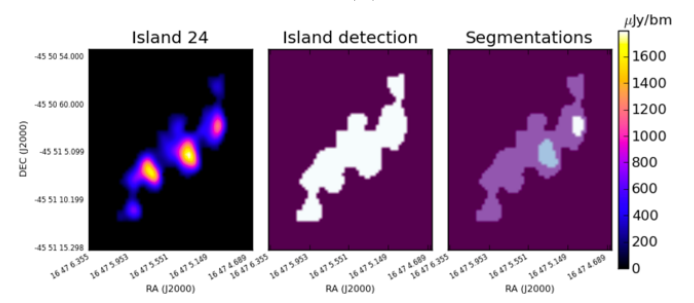

(c)

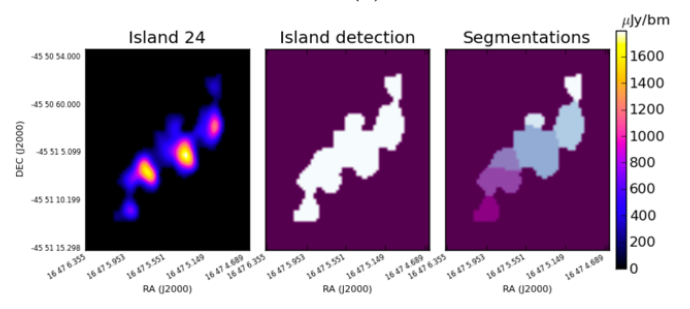

(d)

Fig. 4. SEAC output for WR B from $u-v$ tapered images created from TAPERALMA dataset $(a)$ and TAPER 9 dataset. The non-segmented source detection from SEAC is presented in $(b)$, the result of NOISE segmentation in $(c)$, and the result of GAUSS segmentation in $(d)$.

still difficulties experienced in applying a Gaussian fit due to the extended levels of diffuse radio background throughout the cluster. For sources that were found to be clearly non-Gaussian, a largest angular size (LAS) was determined instead, via visual inspection of the goodness of fit.

Both convolved and deconvolved sizes are given in Table 5, when applicable. For non-Gaussian sources, only the convolved size is given. For sizes determined from a Gaussian fit, errors are taken directly for the convolved sizes, with errors from the associated minimum and maximum ranges of the deconvolved size used to calculate the deconvolved spatial dimension errors. Sources that are fully resolved can all be separated into pointlike sources that fit well to a Gaussian, or sources with significant asymmetric extended emission.

Most of the sources were found to have resolved, extended emission. The large de-convolved sizes that were measured can also be considered in comparison to the prior ALMA observations in F18 where many of the stellar detections were unexpectedly found to be resolved, especially many of the WRs in the cluster. The diffuse radio emission detected throughout the cluster may have impacted the measurements. The impact of the extended emission is discussed further in Sect. 4.

\subsection{Comparison to D10 measurements}

The preliminary results revealed the presence of diffuse emission throughout the cluster. The possible astrophysical origin of this and its impact on our conclusions about sources present in the cluster is discussed further in Sects. 4 and 7. Motivated by this diffuse emission, and as a sanity check on the possible effect of this onto the source fluxes, a consideration of the source size and flux was carried out on the FULL9 dataset in comparison to the previous $8.6 \mathrm{GHz}$ radio observations (D10). This consideration involved the use of altering the user-parameters applied in the segmentation tool in SEAC to adjust the final size of the emission measured for core components of each source. The number of pixels with which flux was measured was adjusted indirectly with the alteration of the user-parameters, so that the relative sizes were found to be as similar in the output as possible, using the D10 images as a reference. This involved the consideration of the relative pixel sizes of each image, and was carried out on a source by source basis.

In general, this comparison showed that we could make conservative measurements of sources by selecting core components of the source from SEAC, with increased fluxes still typically found for sources in the new radio observations. A small number of sources that were exceptions to this were found to be so due to their location within the cluster, where they were in close proximity to much brighter radio sources and surrounded by large extended regions of emission. Other than the effect of larger components being picked up for the total emission regions, including extended components, the increase in flux seen for the FULL9 dataset is likely due to increased $u-v$ coverage, resulting from the use of a wider bandwidth in these more recent ATCA observations. The increased bandwidth will allow for a larger number of $u-v$ visibilities to be sampled, allowing for the FULL9 dataset to be sensitive to a larger number of spatial scales within the same emission region. We can therefore conclude that the D10 and FULL9 datasets provide source fluxes that are broadly consistent with each other.

The comparative fluxes determined from this analysis are listed in Table A.1, with a comparative plot also presented in Fig. B.2. A note was also made of whether sources were particularly isolated with respect to other sources and the diffuse emission, or were located in more crowded regions within the cluster. Where possible, both fluxes from core components and total source fluxes, including an extended component, were measured.

\subsection{Spectral indices}

The spectral indices of all prior known sources were calculated by comparing source fluxes at three frequencies; $5.5 \mathrm{GHz}$, $9 \mathrm{GHz}$, and $100 \mathrm{GHz}$. In order to compare the observations for calculating spectral indices, tapering was applied to datasets of ATCA and ALMA observations, so that only equivalent $u-v$ ranges were considered. However, this led to a reduction in the absolute fluxes found for the sources due to the removal of several short spacings in $u-v$ space from the data considered for the image. The reduction in absolute flux density values found from these images was not considered critical to the spectral index derived, as the final value calculated was reliant on the relative flux values at each frequency, rather than the absolute values. 


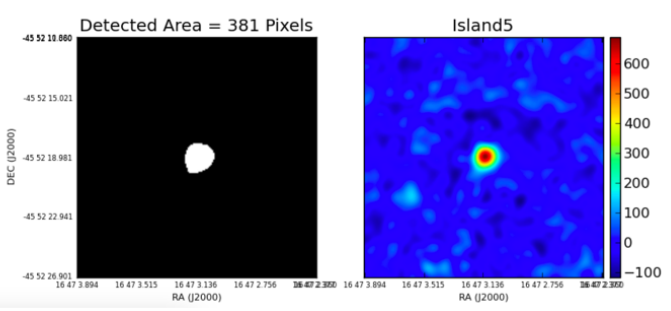

(a)

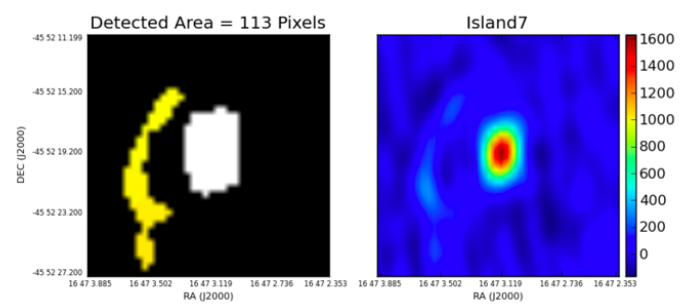

(b)

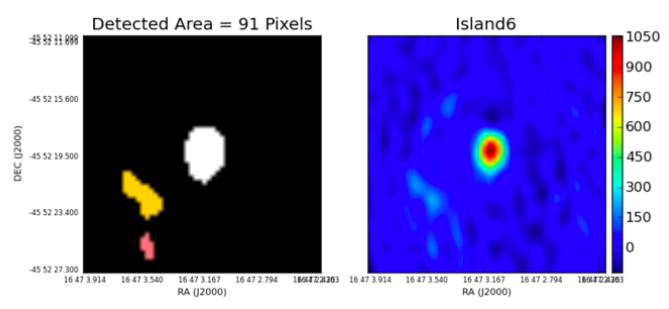

(c)

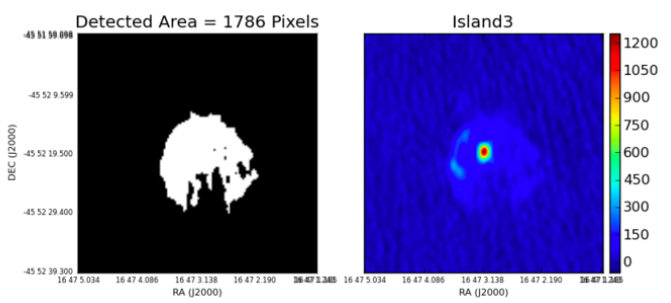

(d)

Fig. 5. SEAC output for W237 from $u-v$ tapered datasets, TAPERALMA $(a)$, TAPER5 $(b)$, and TAPER9 $(c)$, as well as FullConCAT dataset $(d)$.

Using tapered visibilities allowed for similar source sizes to be considered from the ALMA and ATCA observations, as seen in Fig. 5. The W237 images from TAPER5 and TAPER9, as shown in Figs. $5 \mathrm{~b}$ and c, show only the compact bright emission detected for W237, with no inclusion of dimmer more extended emission from the longer spatial scales, that can be seen around W237 in the FULLCONCAT dataset, shown in Fig. 5d. However, an initial run-through of SEAC on these datasets still led to inconsistencies in spectral variance when considering the relationship found between the two radio spectral windows, and the overall relationship calculated in the radio-mm spectral index.

Upon further investigation on a source-by-source basis, the initial inconsistencies in spectral variance were found to be due to differing source sizes determined for the core component of the emission. In order to give the closest comparison possible of the flux when considering the spectral index, a variety of segmentation thresholds were applied to the data, in order to measure core components of the sources. This involved editing the user-defined noise levels in the NOISE segmentation tool, and adjusting the smoothing parameter in the GAUSS segmentation, until the source size determined for known stellar objects with previous detections were as similar in size as possible, across the different datasets (separated by spectral window). As the pixel sizes of the ALMA and ATCA datasets differed, this was taken into consideration when finding smaller source sizes representative of core emission. The revision of comparative fluxes and spectral indices was not found to significantly affect the overall spectral index determined for the sources from the comparison across all three spectral windows at $5.5 \mathrm{GHz}, 9 \mathrm{GHz}$, and $100 \mathrm{GHz}$. However, it did reduce the level of conflict found when comparing the spectral variance across the two radio wavelengths, $5.5 \mathrm{GHz}$ and $9 \mathrm{GHz}$, to the index measured across all three frequency bands.

\section{Extended emission}

One of the main results to become immediately apparent when viewing the images generated by these observations was the large level of diffuse radio emission present throughout the cluster, as seen clearly in Figs. 1-3. The presence of this pervasive diffuse emission calls into question the origin of the material (and therefore the resulting flux measured) surrounding the majority of sources, where the radio emission is found to be resolved and extended. It is unclear whether this material can be truly attributed to these stellar sources due to their stellar wind, or whether the material is actually part of the diffuse background that has become captured by, or is simply interacting with, far more compact nebulae surrounding the sources. If these extended nebulae are truly part of the diffuse background, it is unclear why the majority of this emission is still seen to be directly surrounding the stellar objects, with the most significantly extended structures found around cluster members that are expected to have dense winds and prior periods of extreme mass loss, such as the cool supergiants, hypergiants, and the WR stars. The possible origin of this diffuse emission is discussed further in Sect. 7.

Due to the high levels of diffuse radio emission observed, we had to acknowledge a caveat in our analysis of these observations that radio fluxes measured may include radio emission that has been misattributed to stellar sources. The definitive radio flux densities of the sources, given in Table 3, were calculated from the image generated from the FULLCONCAT dataset, as shown in Fig. 3, where the inclusion of the full set of data allowed for the highest sensitivity.

This diffuse emission may have affected the fluxes measured, the spatial extents found for sources, and all parameters derived from these measurements, including the spectral indices, and the mass-loss rates. We were able to mitigate for the impact of this with the use of conservative flux measurements, and by considering only the core components of emission from sources wherever possible. This is discussed in more detail in Sect. 3.

The spectral indices and mass-loss rates used radio and ALMA measurements in comparison. When calculating the spectral indices, the effect from the extended emission could lead to over-estimates for the radio measurements. As this background flux is believed to be optically-thin in nature, it would be expected to have a flat spectra, and so have a greater impact on the ATCA flux values than on the higher frequency ALMA values. It would also preferentially effect the ATCA radio observations, due to the lower relative fluxes of the stellar sources, if they are expected to emit thermal emission, and be impacted by the lower resolution of the radio observations. Accounting for all possible impacts led to the consideration of source fluxes from comparative source sizes across the ALMA and the ATCA tapered datasets when calculating spectral indices and mass-loss values, as was described in Sect. 3.5. 
We can also consider the possible impact of external ionisation on the resultant spectral indices. Previous observations of externally ionised winds of hot stars, including the wind of the red supergiant (RSG) IRS 7, and the YHG HR 8752, have shown spectral indices that are consistent with values found for internally ionised stellar winds. This indicates that although the stellar winds of these cool evolved stars are ionised by surrounding hot stars (in the case of hot stars in the nearby nuclear cluster for the RSG, and the case of an early B companion for the YHG Stickland \& Harmer 1978), the external ionisation doesn't have a significant effect on the spectral indices (Higgs et al. 1978; Yusef-Zadeh \& Morris 1991).

As the extended emission can be seen to be asymmetric in structure over the cluster, with the strongest illumination orientated towards the cluster centre, it is also of importance to consider whether this would have an impact on the resulting stellar winds. The Pistol star, an LBV located in the Quintuplet cluster, has one hemisphere ionised by its host cluster, though is surrounded by a shell of cold dust, as has been seen in the infra-red (Lau et al. 2014). The spectral index of this star has been found to be consistent with free-free emission from optically thin ionised plasma, which is evidence that even with only part of the shell externally ionised, the end result can be seen to be thermal emission (Yusef-Zadeh et al. 1989).

The result of over-estimated fluxes would lead to flattened spectral indices. This means any impact on the spectral indices would be to make thermal sources appear less thermal. For the majority of sources, especially in the case of the WRs, these objects are already thermal, as shown in Sect. 6.1, and so any removal of this extended emission would only lead to spectral indices that could still be considered to be in line with the canonical value for a thermally-emitting stellar wind. It would also lead to flattened spectral indices for several of the cool hypergiants and supergiants, which are already significantly deviated from the canonical wind value, in line with expectations of composite emission, containing optically thick and thin components. The extended emission would also have led to effects causing over-estimates of the spatial extents and mass-loss rates of sources. In order to counteract any possible impact of this, the mass-loss rates are calculated using only core components of flux from the stellar sources as a conservative estimate, and the resulting mass-loss rates are compared throughout the discussion to rates calculated for analogous sources of each spectral type.

In order to try to quantify the impact of the extended emission on our results, a comparison was carried out between the prior $8.6 \mathrm{GHz}$ D10 observations and the $9 \mathrm{GHz}$ results from the new radio census, with the outcome shown in Table A.1 and Fig. B.2. This was discussed previously in Sect. 3.4, which concluded that the fluxes were broadly consistent between the observations, with slight increases generally found for the new radio results due to the larger $u-v$ coverage resulting in higher sensitivity to more spatial scales. Any decrease in fluxes was found to be due to the location of a source embedded within a diffuse region, and so only a core component could be selected from within the background, resulting in conservative source fluxes measured.

One example of source consideration is the ATCA flux value attributed to one of the most radio luminous sources in the cluster, W9, found to be significantly higher at $9 \mathrm{GHz}$ in the more recent results, with a flux of $30.47 \pm 0.09 \mathrm{mJy}$, in comparison to the core flux component measured by D10, $24.9 \pm 2.5 \mathrm{mJy}$. For the measurement including the extended component, vastly different fluxes were found, with our flux measurement of $80.8 \pm 0.5 \mathrm{mJy}$, versus the D10 detection of $30.5 \pm 3.0 \mathrm{mJy}$. This is due in part to a larger amount of surrounding radio emission
Table 2. Summary of radio detections from FULLCONCAT dataset.

\begin{tabular}{ll}
\hline \hline Category & Source number \\
\hline ATCA+optical & 30 \\
ATCA+ALMA & 30 \\
ATCA-only & 53 \\
\hline Total & 113 \\
\hline WRs & 10 \\
YHGs & 5 \\
RSGs & 4 \\
BSGs & 2 \\
LBV & 1 \\
sgB[e] & 1 \\
OB supergiants & 7 \\
\hline Total & 30 \\
\hline
\end{tabular}

detected around W9. However, it was found that due to the increased sensitivity to a range of spatial scales of emission, despite core components for the FULL9 dataset picked out by SEAC typically resulting in comparatively smaller sizes than the core components selected in D10, the core region measured in the FULL9 still contained a much larger level of flux.

\section{Results of the radio census}

\subsection{Stellar sources}

These radio observations led to 30 radio detections of known stellar sources in Westerlund 1, summarised in Table 2. Out of the 30 stellar sources with confirmed radio fluxes found in FULLCONCAT, five of the ten WR radio detections, and five of the seven detections of $\mathrm{OB}$ supergiants are new radio detections. The WRs newly detected in the radio are WR D and WR G, WN7o stars, WR I, a WN8o star, and WR E and M, both WC9 stars. The OB supergiants with new detections are $\mathrm{W} 10, \mathrm{~W} 18$, and W19, all early B supergiants, and W1031 and W1056, both late O-type giants. Flux densities for stellar sources with optical counterparts are given in Table 3, with flux densities taken from the integrated island flux found by SEAC. Sources with core and total flux components as detected by SEAC are listed with both flux values, specified by the superscript $c$ and $t$ respectively. Plots for detected stellar sources not presented throughout the main body of the paper are shown in Fig B.5.

By investigating the FULL9 dataset, we could compare our radio detections directly to the previous $8.6 \mathrm{GHz}$ radio observations of Westerlund 1 cluster members (D10). Of the 21 sources detected by $\mathrm{D} 10$ at $8.6 \mathrm{GHz}$, all but 1 source is also detected in the FULL9 dataset. The lack of detection for this source, WR V, can be linked to its location embedded within an area of diffuse emission in the cluster (discussed in more detail in Sect. 6.1).

Spectral indices and spectral index limits were determined for all sources where at least one flux density from the tapered datasets TAPERALMA, TAPER5, or TAPER9 could be found. These are presented in Table 4. This table also includes spectral index limits found for the new detections of OB supergiants with no corresponding millimetre detections, with the radio flux density taken from FULLCONCAT due to the faintness of the sources, and using the flux density limit from the full ALMA observations (F18).

Spectral index limits derived from the new radio detections of OB supergiants leads to the conclusion that these stars are binary candidates. The spectral index limits are found to 
Table 3. Flux densities for known sources from FULLCONCAT dataset.

\begin{tabular}{|c|c|c|c|c|}
\hline Source & Spectral type & RA & Dec & Flux $_{\text {FULLCONCAT }}(\mathrm{mJy})$ \\
\hline WR A & $\mathrm{WN} 7 \mathrm{~b}+\mathrm{OB} ?$ & 16478.36412 & -455045.5948 & $\begin{array}{l}0.89 \pm 0.06 \\
1.38 \pm 0.09\end{array}$ \\
\hline WR D & WN7o & 16476.29738 & -455126.6985 & $0.71 \pm 0.05$ \\
\hline WR B & $\mathrm{WN7} 7 \mathrm{O}+\mathrm{OB} ?$ & 16475.34956 & -45515.3995 & $\begin{array}{l}3.38 \pm 0.03^{c} \\
16.53 \pm 0.15^{t}\end{array}$ \\
\hline WR G & WN7o & 16474.05743 & -455123.7000 & $0.34 \pm 0.04$ \\
\hline WR P & WN7o & 16471.61624 & -455145.5984 & $0.05 \pm 0.02^{m}$ \\
\hline WR I & WN80 & 16470.89863 & -455120.6974 & $0.42 \pm 0.02$ \\
\hline WR L & WN9h+OB? & 16474.20100 & -45517.5000 & $0.55 \pm 0.04$ \\
\hline WR S & WN10-11h/BHG & 16472.96652 & -455019.7997 & $0.16 \pm 0.04$ \\
\hline WR E & WC9 & 16476.06807 & -45528.6988 & $0.13 \pm 0.02$ \\
\hline WR F & WC9d+OB? & 16475.20647 & -455225.1996 & $0.29 \pm 0.02$ \\
\hline WR M & WC9d & 16473.94256 & -455138.1000 & $0.24 \pm 0.04$ \\
\hline W16a & $\mathrm{A}^{2} \mathrm{Ia}^{+}$ & 16476.69880 & -455041.0980 & $2.70 \pm 0.11$ \\
\hline W12a & $\mathrm{F} 1 \mathrm{Ia}^{+}$ & 16472.19106 & -455059.3991 & $\begin{array}{l}1.76 \pm 0.03^{c} \\
3.77 \pm 0.07^{t}\end{array}$ \\
\hline W4a & $\mathrm{F} 3 \mathrm{Ia}^{+}$ & 16471.44479 & -455037.4982 & $\begin{array}{l}3.18 \pm 0.04^{c} \\
4.72 \pm 0.07^{t}\end{array}$ \\
\hline W32 & $\mathrm{F} \mathrm{Ia}^{+}$ & 16473.71289 & -455043.8000 & $0.18 \pm 0.04$ \\
\hline W265 & $\mathrm{F} \mathrm{Ia}^{+}$ & 16476.29597 & -454924.2985 & $\begin{array}{l}1.02 \pm 0.04^{c} \\
3.34 \pm 0.11^{t}\end{array}$ \\
\hline W237 & M3Ia & 16473.10953 & -455219.1998 & $\begin{array}{l}1.31 \pm 0.02^{c} \\
9.57 \pm 0.19^{t}\end{array}$ \\
\hline W75 & M4Ia & 16478.93719 & -454958.7933 & $0.34 \pm 0.03$ \\
\hline W20 & M5Ia & 16474.68920 & -455124.2999 & $\begin{array}{l}2.54 \pm 0.03^{c} \\
20.65 \pm 0.23^{t}\end{array}$ \\
\hline W26 & M5-6Ia & 16475.40679 & -455036.5994 & $153.11 \pm 0.17$ \\
\hline W17 & O9Iab & 16476.18210 & -455049.4987 & $\begin{array}{l}0.98 \pm 0.04^{c} \\
2.04 \pm 0.08^{t}\end{array}$ \\
\hline W243 & A2Ia (LBV) & 16477.50460 & -455229.3966 & $1.38 \pm 0.04$ \\
\hline W9 & $\operatorname{sgB}[\mathrm{e}]$ & 16474.14355 & -455031.5000 & $\begin{array}{l}27.38 \pm 0.06^{c} \\
83.14 \pm 0.41^{t}\end{array}$ \\
\hline D09-R1 & BSG & 16479.08253 & -455110.1929 & $\begin{array}{l}1.29 \pm 0.03^{c} \\
5.44 \pm 0.10^{t}\end{array}$ \\
\hline D09-R2 & BSG & 16476.89972 & -455037.1977 & $0.96 \pm 0.06$ \\
\hline W15 & O9Ib & 16476.72735 & -455028.7980 & $1.65 \pm 0.09$ \\
\hline W10 & $\mathrm{B} 0.5 \mathrm{I}+\mathrm{OB}$ & 16473.42580 & -455035.3999 & $0.16 \pm 0.05$ \\
\hline W18 & B0.5Ia & 16475.60787 & -455050.3993 & $0.36 \pm 0.06$ \\
\hline W19 & B1Ia & 16474.86139 & -455057.8998 & $0.08 \pm 0.03$ \\
\hline W1031 & O9III & 16471.96137 & -455057.8988 & $0.90 \pm 0.05$ \\
\hline W1056 & O9.5II & 16478.70904 & -45512.0939 & $0.09 \pm 0.02$ \\
\hline
\end{tabular}

Notes. Flux densities are from the FullConCAT dataset, measured by SEAC (with flood threshold, $\sigma_{\mathrm{f}}=3$, and seed threshold, $\sigma_{\mathrm{s}}=5$ ), with consideration of segmentation where necessary. Flux densities are given in mJy. For extended sources, where possible, core flux densities are denoted by the superscript, $c$, and total flux densities are denoted by the superscript, $t$. A marginal flux detection is indicated with the superscript $m$. Spectral type identifications are taken from Crowther et al. (2006) for the WR stars, Clark et al. (2010) for the YHGs and RSGs, D10 for D09-R1 and D09-R2, and Clark et al. (2019b) for the OB stars.

constrain the spectral indices to negative values, implying the presence of non-thermal emission. These spectral index limits include the cluster members currently categorised as OB supergiants, W15, W18, and W1031, as well as OB supergiants with new radio detections, $\mathrm{W} 10, \mathrm{~W} 19$, and $\mathrm{W} 1056$. This is discussed in more detail in Sect. 6.3.1.

\subsection{Non-stellar sources}

\subsubsection{ALMA sources}

Previous observations made in the millimetre (F18), detected a large number of sources that were not associated with any catalogued stellar sources. Thirty of these objects are now found to have associated detections in the radio. Flux densities are determined, with the positions and fluxes listed in Table A.2, with sources presented in a corresponding plot of the cluster, in Fig. B.3. The number assigned to these sources is the FCP18 source number, as given in F18.

One of the sources can clearly be attributed to a knot of emission associated with the extended nebulae around the YHG W4a. Four of the sources are found to be aligned with a significant area of extended emission to the west of Wd1 (to the RHS side of the figure). A large number of the rest of the sources can be found clustered within other regions of diffuse radio background 
Table 4. Spectral indices and index limits of known stellar sources.

\begin{tabular}{|c|c|c|c|c|}
\hline Source & Flux $_{\text {TAPER5 }}(\mathrm{mJy})$ & Flux $_{\text {TAPER } 9}(\mathrm{mJy})$ & Flux $_{\text {TAPERALMA }}(\mathrm{mJy})$ & Spectral index $(\alpha)$ \\
\hline WR O & $<0.10$ & $<0.10$ & $0.28 \pm 0.04$ & $>0.37$ \\
\hline WR U & $<0.19$ & $<0.15$ & $0.17 \pm 0.05$ & $>-0.01$ \\
\hline WR Q & $<0.12$ & $<0.10$ & $0.18 \pm 0.05$ & $>0.17$ \\
\hline WR A & $0.62 \pm 0.08$ & $0.94 \pm 0.05$ & $3.89 \pm 0.22$ & $0.61 \pm 0.04$ \\
\hline WR D & $<0.17$ & $0.17 \pm 0.05$ & $0.58 \pm 0.07$ & $0.50 \pm 0.16$ \\
\hline WR B & $3.51 \pm 0.12$ & $2.96 \pm 0.09$ & $2.62 \pm 0.23$ & $-0.10 \pm 0.03$ \\
\hline WR G & $<0.16$ & $0.13 \pm 0.04$ & $0.72 \pm 0.08$ & $0.71 \pm 0.14$ \\
\hline WR P & $<0.12$ & $<0.12$ & $0.50 \pm 0.06$ & $>0.52$ \\
\hline WR I & $0.16 \pm 0.04$ & $0.47 \pm 0.04$ & $2.02 \pm 0.11$ & $0.79 \pm 0.08$ \\
\hline WR V & $<0.24$ & $<0.28$ & $0.90 \pm 0.09$ & $>0.47$ \\
\hline WR L & $0.24 \pm 0.06$ & $0.48 \pm 0.04$ & $3.37 \pm 0.18$ & $0.88 \pm 0.07$ \\
\hline WR S & $<0.13$ & $<0.09$ & $0.36 \pm 0.07$ & $>0.42$ \\
\hline W13 & $<0.20$ & $<0.21$ & $0.18 \pm 0.05$ & $>-0.05$ \\
\hline WR K & $<0.16$ & $<0.14$ & $0.16 \pm 0.05$ & $>0.02$ \\
\hline WR E & $<0.16$ & $<0.10$ & $0.81 \pm 0.06$ & $>0.66$ \\
\hline WR F & $<0.12$ & $0.37 \pm 0.04$ & $1.26 \pm 0.09$ & $0.51 \pm 0.07$ \\
\hline WR C & $<0.17$ & $<0.11$ & $0.22 \pm 0.04$ & $>0.15$ \\
\hline WR H & $<0.12$ & $<0.09$ & $0.28 \pm 0.06$ & $>0.35$ \\
\hline WR M & $<0.16$ & $0.11 \pm 0.03$ & $0.39 \pm 0.05$ & $0.53 \pm 0.12$ \\
\hline W16a & $1.59 \pm 0.11$ & $1.67 \pm 0.11$ & $1.23 \pm 0.15$ & $-0.10 \pm 0.05$ \\
\hline W12a & $1.77 \pm 0.05$ & $1.70 \pm 0.08$ & $0.78 \pm 0.13$ & $-0.30 \pm 0.07$ \\
\hline W4 & $1.85 \pm 0.04$ & $1.84 \pm 0.04$ & $1.78 \pm 0.16$ & $-0.01 \pm 0.04$ \\
\hline W265 & $1.00 \pm 0.04$ & $0.94 \pm 0.08$ & - & $-0.13 \pm 0.23$ \\
\hline W237 & $1.07 \pm 0.06$ & $1.03 \pm 0.05$ & $1.05 \pm 0.08$ & $0.00 \pm 0.04$ \\
\hline W75 & $<0.13$ & $0.23 \pm 0.05$ & $0.22 \pm 0.05$ & $-0.01 \pm 0.12$ \\
\hline W20 & $2.23 \pm 0.07$ & $2.10 \pm 0.06$ & $1.99 \pm 0.16$ & $-0.03 \pm 0.03$ \\
\hline W26 & $106.3 \pm 0.2$ & $117.7 \pm 0.4$ & $103.3 \pm 5.2$ & $-0.02 \pm 0.02$ \\
\hline W17 & $0.81 \pm 0.09$ & $0.86 \pm 0.09$ & $0.76 \pm 0.12$ & $-0.03 \pm 0.07$ \\
\hline W46a & $<0.11$ & $<0.09$ & $0.18 \pm 0.04$ & $>0.21$ \\
\hline W243 & $0.82 \pm 0.05$ & $1.54 \pm 0.07$ & $9.69 \pm 0.49$ & $0.82 \pm 0.03$ \\
\hline W7 & $<0.09$ & $<0.11$ & $0.20 \pm 0.04$ & $>0.27$ \\
\hline W9 & $22.10 \pm 0.07$ & $30.34 \pm 0.11$ & $158.6 \pm 7.9$ & $0.68 \pm 0.02$ \\
\hline D09-R1 & $0.82 \pm 0.07$ & $0.96 \pm 0.07$ & $0.85 \pm 0.14$ & $-0.01 \pm 0.07$ \\
\hline D09-R2 & $0.54 \pm 0.06$ & $0.59 \pm 0.07$ & $0.51 \pm 0.07$ & $-0.03 \pm 0.07$ \\
\hline Source & & Flux $_{\text {FULLCONCAT }}(\mathrm{mJy})$ & $\operatorname{Flux}_{\text {ALMA }}(\mathrm{mJy})$ & Spectral index $(\alpha)$ \\
\hline W15 & & $1.65 \pm 0.09$ & $<0.10$ & $<-1.15$ \\
\hline W10 & & $0.16 \pm 0.05$ & $<0.15$ & $<-0.04$ \\
\hline W18 & & $0.36 \pm 0.06$ & $<0.127$ & $<-0.43$ \\
\hline W19 & & $0.08 \pm 0.03$ & $<0.104$ & $<0.10$ \\
\hline W1031 & & $0.90 \pm 0.05$ & $<0.09$ & $<-0.95$ \\
\hline W1056 & & $0.09 \pm 0.02$ & $<0.100$ & $<0.02$ \\
\hline
\end{tabular}

Notes. Top panel shows spectral indices and index limits derived from TAPERALMA, TAPER5, and TAPER9 for stellar sources with an ALMA detection. For extended sources, core fluxes are used. Detection limits on ATCA data were measured by determining the rms, $\sigma$, in the local area of each source that was not detected, using a surrounding circular region of six arcseconds, and taking flux density limits as $3 \sigma$. Bottom panel shows spectral index limits using flux densities from FuLLCONCAT dataset for stellar sources with no ALMA detection. Millimetre flux limits are taken from Table A.2 of F18, where the detection limit is $3 \sigma$, with $\sigma$ the rms from a circular region of two arcseconds around the optical positions of the stars.

or nearby radio luminous known stellar sources, such as W9, WR B, and D09-R1. At least seven of the sources detected were found to be in isolated positions and not linked to any known stellar sources in the cluster.

Fifteen of these sources were strong enough in the radio for spectral indices to be calculated from the tapered datasets, TAPER5, TAPER9, and TAPERALMA, as shown in Table A.3. Overwhelmingly, all of these sources are found to have clearly non-thermal spectral indices. Some of the sources that are seen to have a negative spectral index are clearly embedded within a larger diffuse radio background. This indicates that shocks within the cluster wind may be responsible for these knots of emission (Bell 1978). Some of the uncategorised sources are in the near vicinity of stellar sources and the impact of extended emission from these nearby stars may influence the spectral behaviour observed (Yusef-Zadeh et al. 2003).

However, some sources cannot be linked to either of these external influences, and so must experience a non-thermal spectral index for another reason, most notably in the case of sources FCP18-16, -22, -27, and -95. Possible explanations for this spectral behaviour are the presence of synchrotron emission in the radio resulting from an as of yet undetected binary stellar 
Table 5. Spatial extents measured from FuLlConCAT dataset.

\begin{tabular}{|c|c|c|c|c|c|c|c|}
\hline \multirow[t]{2}{*}{ Source } & \multirow[t]{2}{*}{ RA (16 47) } & \multirow[t]{2}{*}{$\operatorname{Dec}(-45)$} & \multirow[t]{2}{*}{ Offset } & \multicolumn{2}{|c|}{ Size convolved } & \multicolumn{2}{|c|}{ Size deconvolved } \\
\hline & & & & Major axis $\left({ }^{\prime \prime}\right)$ & Minor axis (" & Major axis $\left({ }^{\prime \prime}\right)$ & Minor axis $\left({ }^{\prime \prime}\right)$ \\
\hline WR A & 8.36412 & 5045.5948 & 0.47 & $2.49 \pm 0.07$ & $1.34 \pm 0.04$ & $1.82 \pm 0.11$ & $0.89 \pm 0.08$ \\
\hline WR D & 6.29738 & 5126.6985 & 0.65 & 4.96 & LAS & - & - \\
\hline WR B & 5.34956 & 515.3995 & 0.40 & $\begin{array}{l}3.23 \pm 0.03 \\
6.98\end{array}$ & $\begin{array}{l}2.32 \pm 0.02 \\
\text { LAS }\end{array}$ & $\begin{array}{l}2.77 \pm 0.04 \\
-\end{array}$ & $\begin{array}{l}2.06 \pm 0.03 \\
-\end{array}$ \\
\hline WR G & 4.05743 & 5123.7000 & 1.57 & $3.05 \pm 0.19$ & $1.19 \pm 0.08$ & $2.53 \pm 0.24$ & $0.63 \pm 0.18$ \\
\hline WR I & 0.89863 & 5120.6974 & 0.22 & $1.86 \pm 0.07$ & $1.05 \pm 0.04$ & $0.80 \pm 0.21$ & - \\
\hline WR L & 4.20100 & 517.5000 & 0.14 & $1.76 \pm 0.05$ & $1.13 \pm 0.03$ & $0.55 \pm 0.34$ & $0.39 \pm 0.17$ \\
\hline WR S & 2.96652 & 5019.7997 & 0.25 & $2.25 \pm 0.26$ & $1.96 \pm 0.22$ & $1.74 \pm 0.51$ & $1.42 \pm 0.65$ \\
\hline WR E & 6.06807 & 528.6988 & 0.54 & $1.78 \pm 0.19$ & $1.10 \pm 0.12$ & $0.51 \pm 0.53$ & $0.47 \pm 0.41$ \\
\hline WR F & 5.20647 & 5225.1996 & 0.25 & $1.89 \pm 0.10$ & $1.06 \pm 0.06$ & $0.81 \pm 0.30$ & $0.37 \pm 0.28$ \\
\hline WR M & 3.94256 & 5138.1000 & 0.36 & $2.18 \pm 0.17$ & $1.61 \pm 0.12$ & $1.49 \pm 0.33$ & $1.10 \pm 0.4$ \\
\hline$\overline{\mathrm{W} 16 \mathrm{a}}$ & 6.69880 & 5041.0980 & 1.37 & $3.66 \pm 0.09$ & $2.41 \pm 0.06$ & $3.25 \pm 0.10$ & $2.18 \pm 0.07$ \\
\hline $\mathrm{W} 12 \mathrm{a}$ & 2.19106 & 5059.3991 & 0.61 & $\begin{array}{l}2.39 \pm 0.03 \\
4.81\end{array}$ & $\begin{array}{l}2.05 \pm 0.03 \\
\text { LAS }\end{array}$ & $\begin{array}{l}1.80 \pm 0.05 \\
-\end{array}$ & $\begin{array}{l}1.67 \pm 0.06 \\
-\end{array}$ \\
\hline W4a & 1.44479 & 5037.4982 & 0.47 & $\begin{array}{l}2.35 \pm 0.02 \\
6.91\end{array}$ & $\begin{array}{l}1.55 \pm 0.01 \\
\text { LAS }\end{array}$ & $\begin{array}{l}1.62 \pm 0.03 \\
-\end{array}$ & $\begin{array}{l}1.19 \pm 0.02 \\
-\end{array}$ \\
\hline W32 & 3.71289 & 5043.8000 & 0.50 & $1.78 \pm 0.09$ & $1.04 \pm 0.05$ & $0.50 \pm 0.4$ & $0.31 \pm 0.28$ \\
\hline W265 & 6.29597 & 4924.2985 & 0.72 & $\begin{array}{l}2.46 \pm 0.05 \\
12.59\end{array}$ & $\begin{array}{l}1.92 \pm 0.04 \\
\text { LAS }\end{array}$ & $\begin{array}{l}1.94 \pm 0.09 \\
-\end{array}$ & $\begin{array}{l}1.44 \pm 0.10 \\
-\end{array}$ \\
\hline W237 & 3.10953 & 5219.1998 & 0.43 & $\begin{array}{l}2.60 \pm 0.04 \\
15.36\end{array}$ & $\begin{array}{l}1.94 \pm 0.03 \\
\text { LAS }\end{array}$ & $\begin{array}{l}1.97 \pm 0.06 \\
-\end{array}$ & $\begin{array}{l}1.64 \pm 0.04 \\
-\end{array}$ \\
\hline W75 & 8.93719 & 4958.7933 & 0.22 & $2.74 \pm 0.15$ & $1.46 \pm 0.08$ & $2.16 \pm 0.19$ & $1.05 \pm 0.13$ \\
\hline W20 & 4.68920 & 5124.2999 & 0.50 & $\begin{array}{l}2.89 \pm 0.03 \\
21.05\end{array}$ & $\begin{array}{l}2.21 \pm 0.02 \\
\text { LAS }\end{array}$ & $2.35 \pm 0.04$ & $\begin{array}{l}1.96 \pm 0.03 \\
-\end{array}$ \\
\hline W26 & 5.40679 & 5036.5994 & 0.18 & 17.04 & LAS & - & - \\
\hline$\overline{\mathrm{W} 17}$ & 6.18210 & 5049.4987 & 0.76 & $2.52 \pm 0.05$ & $2.04 \pm 0.04$ & $2.18 \pm 0.13$ & $1.38 \pm 0.11$ \\
\hline W243 & 7.50460 & 5229.3966 & 0.25 & $1.75 \pm 0.02$ & $1.00 \pm 0.01$ & $0.40 \pm 0.11$ & - \\
\hline W9 & 4.14355 & 5031.5000 & 0.58 & $\begin{array}{l}2.212 \pm 0.001 \\
9.39\end{array}$ & $\begin{array}{l}1.235 \pm 0.001 \\
\text { LAS }\end{array}$ & $\begin{array}{l}1.405 \pm 0.002 \\
-\end{array}$ & $\begin{array}{l}0.735 \pm 0.001 \\
-\end{array}$ \\
\hline D09-R1 & 9.08253 & 5110.1929 & 0.07 & $\begin{array}{l}2.37 \pm 0.03 \\
12.85\end{array}$ & $\begin{array}{l}1.88 \pm 0.02 \\
\text { LAS }\end{array}$ & $\begin{array}{l}1.92 \pm 0.05 \\
-\end{array}$ & $\begin{array}{l}1.24 \pm 0.07 \\
-\end{array}$ \\
\hline D09-R2 & 6.89972 & 5037.1977 & 0.07 & $2.13 \pm 0.05$ & $1.49 \pm 0.04$ & $1.45 \pm 0.10$ & $0.86 \pm 0.13$ \\
\hline$\overline{\mathrm{W} 15}$ & 6.72735 & 5028.7980 & 1.40 & $3.95 \pm 0.10$ & $1.61 \pm 0.04$ & $3.55 \pm 0.12$ & $1.27 \pm 0.06$ \\
\hline W10 & 3.42580 & 5035.3999 & 1.15 & $2.55 \pm 0.22$ & $1.41 \pm 0.12$ & $1.90 \pm 0.23$ & $0.98 \pm 0.22$ \\
\hline W18 & 5.60787 & 5050.3993 & 1.08 & $3.86 \pm 0.67$ & $1.79 \pm 0.31$ & $3.47 \pm 0.8$ & $1.49 \pm 0.4$ \\
\hline W19 & 4.86139 & 5057.8998 & 1.19 & $3.75 \pm 0.16$ & $2.39 \pm 0.10$ & $3.35 \pm 0.19$ & $2.15 \pm 0.13$ \\
\hline W1031 & 1.96137 & 5057.8988 & 1.55 & $2.90 \pm 0.13$ & $2.53 \pm 0.11$ & $2.73 \pm 0.15$ & $1.87 \pm 0.17$ \\
\hline W1056 & 8.70904 & 512.0939 & 1.01 & $2.90 \pm 0.28$ & $1.28 \pm 0.12$ & $2.36 \pm 0.36$ & $0.74 \pm 0.29$ \\
\hline
\end{tabular}

Notes. Spatial extents are given in terms of convolved and deconvolved sizes. Gaussian fits were applied to the stellar sources using the task JMFIT in the software package AIPS. When Gaussian fits could not be applied, a LAS was found instead.

system, where there is an interacting shock between two stellar winds. Another possible explanation is that the knots of radio and millimetre emission could be of extra-galactic origin rather than from Wd1 itself, though this likelihood was discussed and largely dismissed previously in F18.

\subsubsection{ATCA-only sources}

Alongside the detection of previously observed sources in other wave-bands, 53 additional sources of radio emission are detected through the use of SEAC, listed in Table A.4, with the source number assigned from the full SEAC output list, ordered by right ascension (RA). A conservative limit in determining the number of these radio-only sources meant that only knots of radio emission detected without the use of the segmentation tools within SEAC were considered. Some of these sources may be related to extended radio emission from nearby radio luminous sources, including HA19-23 in the nearby vicinity of W12a, and HA19-43 in the close vicinity of the large extended tail of W20. A large number can also be seen (as displayed in Fig. B.4) to be in isolated regions away from any of the previously determined sources in Wd1. Additionally, many of these isolated radio sources display a geometry that indicate the emission cannot be considered an extension to other known sources, due to the compact, point-source like morphology of the emission, including examples such as HA19-65 to the north of the cluster, HA19-3 and HA19-4 to the west of the cluster, and HA19-85, -88 , and -89 to the east (see Fig. B.4). 


\section{Stellar sources}

\subsection{Wolf-Rayet stars}

Westerlund 1 contains 24 known WR stars, comprising almost $4 \%$ of the galactic population (online catalogue, Crowther 2019). Ten WR stars are detected, with five WRs not previously detected in the radio. WR stars are detected across a variety of subtypes, with many late-type WN stars detected (WN7, WN8, WN9), as well as half of the WC WRs in the cluster. Four of the WN stars detected have prior evidence suggesting they may be binary systems, and all of the detected WC stars detected have some evidence of binarity from other observations. Of the 24 WR stars present in Westerlund 1, three were outside of the field of view, meaning that almost half of the available WR stars are detected with associated radio emission (ten detections and one marginal detection of 21 available WR stars).

The star WR P is detected in the ATCA observations, but this result is not presented in Table 3 as it was a marginal detection, with a flux of $0.05 \pm 0.02 \mathrm{mJy}$. Due to a low signal to noise ratio $(\mathrm{S} / \mathrm{N})$ value of $\sim 2.8$, it cannot be listed as a confirmed source. It can be noted that a caveat arose, that with a slightly different defined background noise level (for example, by considering a sub-image created of this source from the full field of view, which leads to a different absolute cell-size measured in generating the background noise level), the flux is able to reach above the $\mathrm{S} / \mathrm{N}$ limit of $\sim 3$.

Of the WR stars not detected but within the central field of view, many of these are located in a nearby proximity to a stellar source with high levels of surrounding extended radio emission. Obscuration from this surrounding emission is highly likely so that any radio flux emitted by the fainter stars is not separable from the extended emission. This is a likely cause of the nondetection of WR V, located close to W9, one of the most luminous radio sources present in the cluster. At the location of WR $\mathrm{V}$, the peak flux is found to be $0.3 \mathrm{mJy}$, with a mean value over the immediate surrounding area of the source of $\sim 0.1 \mathrm{mJy}$, on the same order as the level of $3 \sigma$ in that region, with $\sigma$ the rms value of the local spatial region. This is especially of note considering this source was detected in previous radio observations (D10).

Other sources affected by this include WR U, located near to W16a, WR R, located near to W26, and WR J, located near W12a. The other WRs not detected by ATCA, WR W, O, Q, K, $\mathrm{C}$, and $\mathrm{H}$, may emit radio fluxes, but any emission is too faint to be detected above the noise level of the observations. This is especially likely if we assume a thermal spectral index for these sources.

Fluxes of the WRs from the FULL9 dataset are compared to D10, as shown in Table A.1. Of sources detected in both datasets, consistent fluxes are found for all sources that were not surrounded by extended emission, or in the proximity of nearby radio luminous sources. For sources surrounded by extended emission, the conservative choice of flux determination on these sources (as discussed in Sect. 3), lead to lower flux values, as seen for the source WR S. In other cases, the fluxes are found to be typically slightly higher, due to the increased sensitivity of the more recent observations.

Spectral indices and limits are determined from the tapered datasets, TAPER5, TAPER9, and TAPERALMA, with spectral index values found for eight WRs and spectral index limits found for 11 WRs. The majority of the WR stars are found to have a spectral index in line with the canonical value for a thermal stellar wind. This indicates that even though a majority of the WRs detected have evidence of binarity from other observations
(Clark et al. 2019b), thermal emission is still the dominant mechanism from the outer wind regions. Any colliding winds present must be located at close separations, likely inside of the radio photospheres of the stars. Spectral index limits calculated for the other WRs indicated flattened or positive spectral indices for all sources, consistent with expectations of single stars with thermal wind emission.

Out of the WRs, only WR B is found to have a negative spectral index, found to be $-0.10 \pm 0.03$ as shown in Table 4 . This indicates the presence of non-thermal emission. The source WR B also shows the most complex morphology of the WRs, and is surrounded by a large region of extended emission, as seen in Fig. 6a. The core component of the source was used to determine the spectral index. The negative spectral index determined, $\alpha=-0.10$, is in line with values found in previous millimetre and radio comparisons $(\mathrm{F} 18)$.

The star WR A, as shown in Fig. 6b, is found to have a positive spectral index of $0.61 \pm 0.04$. This revised spectral index is flatter than the previous spectral index found in F18, more in line with the canonical value for partially optically thick free-free emission. Figure $6 \mathrm{c}$ shows WR D, which is found to have a slightly flattened positive spectral index of $\sim 0.50$, also indicating partially optically thick and thin emission. A revised spectral index limit on WR V, $\alpha>0.47$, is consistent with leading to the canonical value for a stellar wind, containing partially optically thick and thin emission. This is in rough agreement with the radio-millimetre spectral index found in F18.

The source WR $\mathrm{G}$ is found to have a highly thermal spectral index $\alpha \sim 0.71$, and the WN8 and WN9+OB? subtypes WR I and WR $L$ are found to have spectral indices $\sim 0.79$ and $\sim 0.88$ respectively. These values are suggestive of a partially optically thick stellar wind with the increased optically thick component possibly due to colliding wind regions. This is because for close in binaries with short period, the material from the wind collision zone (WCZ) can become cooled and cause optically thick thermal emission to dominate the spectrum, causing a steepening of the index over the mm-radio continuum (Pittard 2010; Stevens et al. 1992). The suggestion is supported by previous evidence of binarity for sources WR G and L, although WR I has no current evidence indicative of binarity.

A detection is made for the WN10-11h/BHG WR S, but it is not bright enough to be seen in the TAPER9 or TAPER5 datasets, so can only be given a spectral index limit. The spectral index limit, $\alpha>0.42$, is consistent with the canonical stellar wind value, suggestive of partially optically thick emission, and is higher than the previous radio-millimetre spectral index value determined in F18. The flux value for WR S, much fainter than in the D10 observations, was likely affected by its proximity to the radio luminous object $\mathrm{W} 9$.

The WC9d+OB star WR F and the WC9d star WR M both have spectral indices slightly flattened with comparison to the canonical stellar wind value, of $\sim 0.51$ and $\sim 0.53$ respectively. This could be due to a higher presence of optically thin or non-thermal components, and is likely related to the binarity of these sources. Spectral index limits are also derived for a further 11 WR stars, all in line with either a slightly flattened spectral index or the canonical wind spectral index value. The WolfRayet stars WR E, C, and $\mathrm{H}$ are all binary candidates, but no evidence is found of a significant non-thermal component for these sources. Nine of the WRs, with definitive flux detections in the radio, only excepting WR $\mathrm{B}$, are found to have spectral indices and spectral index limits in line with the value expected for stellar wind emission. Therefore, the stellar fluxes of these WRs could be used to determine their mass-loss rates. 

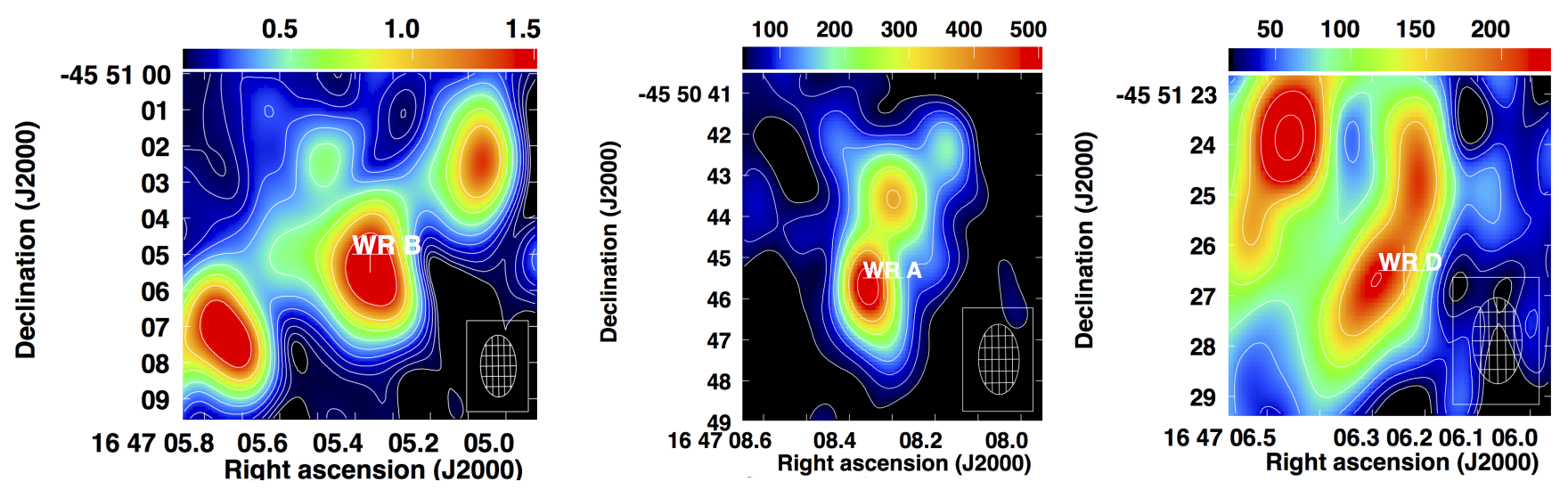

Fig. 6. Non-primary beam corrected images of WR B, A, and D, from FULLCONCAT dataset. Contours are plotted at 1, 1.4, 2, 2.8, 4, 5.7, 8, 11.3, $16,22.6,32,45.25,64,90.50 \times \sigma$, the local rms value.

Table 6. Mass-loss rate determinations of WRs.

\begin{tabular}{lllll}
\hline \hline \multirow{2}{*}{ Source } & \multirow{2}{*}{ Spectral type } & \multicolumn{3}{c}{ ATCA $\dot{M} f_{\mathrm{cl}}^{\frac{1}{2}}$} \\
\cline { 3 - 5 } & & All & $5.5 \mathrm{GHz}$ & \multicolumn{1}{c}{$\left(M_{\odot} \mathrm{yr}^{-1}\right)$} \\
\hline WR A & WN7b+OB? & $7.02 \pm 0.35 \mathrm{e}-05$ & - & $4.01 \pm 2.50 \mathrm{e}-05$ \\
WR D & WN7o & $5.92 \pm 0.31 \mathrm{e}-05$ & $2.97 \pm 0.74 \mathrm{e}-05$ & $3.09 \pm 0.41 \mathrm{e}-05$ \\
WR G & WN7o & $3.41 \pm 0.30 \mathrm{e}-05$ & - & - \\
WR I & WN8o & $3.10 \pm 0.11 \mathrm{e}-05$ & $1.62 \pm 0.32 \mathrm{e}-05$ & $2.96 \pm 0.15 \mathrm{e}-05$ \\
WR L & WN9h+OB? & $2.72 \pm 0.15 \mathrm{e}-05$ & $1.55 \pm 0.32 \mathrm{e}-05$ & $2.35 \pm 0.16 \mathrm{e}-05$ \\
WR S & WN10-11h/BHG & $0.63 \pm 0.12 \mathrm{e}-05$ & - & $0.34 \pm 0.10 \mathrm{e}-05$ \\
WR E & WC9 & $3.62 \pm 0.42 \mathrm{e}-05$ & - & $2.90 \pm 0.40 \mathrm{e}-05$ \\
WR F & WC9d+OB? & $6.62 \pm 0.34 \mathrm{e}-05$ & $3.62 \pm 0.74 \mathrm{e}-05$ & $6.76 \pm 0.44 \mathrm{e}-05$ \\
WR M & WC9d & $5.74 \pm 0.71 \mathrm{e}-05$ & - & $3.84 \pm 0.72 \mathrm{e}-05$ \\
\hline
\end{tabular}

Notes. Mass-loss rates are calculated from fluxes given by the FULLCONCAT, FULL5 and FULL9 datasets. These mass-loss rates are calculated for objects where an approximation of a thermally emitting stellar wind is considered valid, using the Wright and Barlow equation (Wright \& Barlow 1975).

\subsubsection{Mass-loss rates}

For all sources with spectral indices found to be consistent with optically thick or thin free-free emission $(\alpha>0)$, mass-loss rates were determined from their associated fluxes. The Wright and Barlow equation was applied to determine radio mass-loss rates (Wright \& Barlow 1975). This equation includes the assumptions that the stellar wind is spherically symmetric and only emits thermal free-free emission. The mass-loss rate, $\dot{M}$ (combined with the clumping factor, $f_{\mathrm{cl}}$ ) can be determined from the source flux as follows,

$\dot{M} f_{\mathrm{cl}}^{\frac{1}{2}}=0.095 \times \frac{\mu v_{\infty} S_{v}^{\frac{3}{4}} D^{\frac{3}{2}}}{Z \gamma^{\frac{1}{2}} g_{\mathrm{ff}}^{\frac{1}{2}} \nu^{\frac{1}{2}}} \quad M_{\odot} \mathrm{yr}^{-1}$,

where $\dot{M}$ is the mass-loss rate in $M_{\odot} \mathrm{yr}^{-1}$. The parameters involved include $\mu$, the mean molecular weight per ion, $v_{\infty}$, the terminal velocity of the wind (in $\mathrm{km} \mathrm{s}^{-1}$ ), $S_{v}$, the observed flux (in mJy), measured at the frequency $v$ (in Hz), $D$, the distance (in kpc), $Z$, the ratio of electron to ion density, and $\gamma$, the mean number of electrons per ion. The calculation of the massloss rate also requires the calculation of $g_{\mathrm{ff}}$, the gaunt factor, defined by,

$g_{\mathrm{ff}} \sim 9.77\left(1+0.13 \log \left(T_{\mathrm{e}}^{\frac{3}{2}} / v \sqrt{\left(\overline{Z^{2}}\right)}\right)\right)$,

where $T_{\mathrm{e}}$ is the electron temperature (Leitherer \& Robert 1991). The clumping factor, $f_{\mathrm{cl}}$, is defined by $f_{\mathrm{cl}}=\left\langle\rho^{2}\right\rangle /\langle\rho\rangle^{2}$, where $\rho$ is the density of the wind. This demonstrates the underlying relationship between the mass-loss rate derived from the wind and the intrinsic density. If $f_{\mathrm{cl}}$ is taken to be 1 , it represents a smooth-wind, with larger values indicating the presence of structure, suggesting a clumped wind.

For the different stellar types considered, assumptions can be made about the various parameters, $T_{\mathrm{e}}, Z, \mu$ and $\gamma$. Many of these follow the assumptions made in F18. For OB stars, $\mu$ was taken to be 1.4 , and $Z$ and $\gamma$ were both taken to be 1.0. This relates to a chemistry where $\mathrm{H}$ is fully ionised, the dominant form of $\mathrm{He}$ is singly ionised, and the $\mathrm{He}$ abundance is $n_{\mathrm{He}} / n_{\mathrm{H}}=0.1$. The values of $Z$ and $\gamma$ were also taken to be 1.0 for WRs, and $\mu$ was taken as 4.0 for WN6 and earlier spectral types, with 2.0 used for spectral types later than WN6, and 4.7 used for WC8 and WC9 stars. For RSGs, LBVs, and YHGs, $\mu$ was assumed to be $1.4, Z$ was taken to be 0.9 , and $\gamma$ was taken as 0.8 . For all early-type hot stars, $T_{\mathrm{e}}$ was taken to be $0.5 \times T_{\text {eff }}$, where $T_{\text {eff }}$ is the effective temperature of the star. For cool stars, including the YHGs and RSGs, $T_{\mathrm{e}}$ was taken to be $10000 \mathrm{~K}$. The distance, $D$, was assumed to be $5 \mathrm{kpc}$, as justified in the Introduction.

Mass-loss rates were calculated for nine of the 11 detected WRs and are presented in Table 6 . The rates were determined for WRs where the spectral indices or index limits of the stars indicated optically thick or thin emission, in line with thermal stellar winds. The absolute mass-loss rates are calculated for the FULLCONCAT, FULL9, and FULL5 datasets. The majority of these sources show consistency with the expected value for WRs, of $\sim 10^{-5} M_{\odot} \mathrm{yr}^{-1}$. 
A slightly lower value is determined for WR S, though this could be due to the possibility that WR $S$ is believed to be a post-interaction product of a previously close binary, and so has already experienced extra stripping of its outer layers during that phase. Our value for WR S, $\dot{M} f_{\mathrm{cl}}^{\frac{1}{2}} 6.3 \pm 1.2 \times 10^{-6} M_{\odot} \mathrm{yr}^{-1}$, is larger than determined by previous modelling of this object, where $\dot{M} f_{\text {cl }}^{\frac{1}{2}}$ was found to be $2.16 \times 10^{-6} M_{\odot} \mathrm{yr}^{-1}$ (Clark et al. 2014), but is consistent with the measurement from millimetre observations of $5.18 \times 10^{-6} M_{\odot} \mathrm{yr}^{-1}$ (F18).

As WR B is found to have a negative spectral index, this indicates significant non-thermal emission associated with the source, and so the approximation for a thermally emitting stellar wind is not valid for this star. An estimate of $\dot{M} f_{\mathrm{cl}}^{\frac{1}{2}}$ for the WR B flux in the FULLCONCAT dataset was still made, found to be $\sim 1.90 \times 10^{-4} M_{\odot} \mathrm{yr}^{-1}$. This is much higher than the rest of the cohort, supporting the conclusion that this source cannot be realistically approximated with a purely thermally emitting stellar wind.

The radio flux of WR A gives an $\dot{M} f_{\text {cl }}^{\frac{1}{2}}$ value, $7.02 \times$ $10^{-5} M_{\odot} \mathrm{yr}^{-1}$, consistent within uncertainties to the value found in the millimetre (F18) of $7.19 \times 10^{-5} M_{\odot} \mathrm{yr}^{-1}$. As WR A is found to have extended emission, only the flux from its core component is used to calculate the mass-loss rate. As a core component could not be resolved for this source at $5.5 \mathrm{GHz}, \dot{M} f_{\mathrm{cl}}^{\frac{1}{2}}$ values were only determined for the FULLCONCAT and FULL9 datasets.

The star WR D is found to have a $\dot{M} f_{\mathrm{cl}}^{\frac{1}{2}}$ value of $5.92 \pm 0.31 \times 10^{-5} M_{\odot} \mathrm{yr}^{-1}$, larger than the $\mathrm{mm}$ value of $2.07 \times 10^{-5} M_{\odot} \mathrm{yr}^{-1}$ (F18). A larger mass-loss rate is also calculated for WR $G$ from the radio fluxes, with a value of $3.41 \pm 0.30 \times 10^{-5} M_{\odot} \mathrm{yr}^{-1}$ from the radio observations, in contrast to the $\mathrm{mm}$ value of $2.22 \times 10^{-5} M_{\odot} \mathrm{yr}^{-1}$ (F18). This was also the case for WR M, where the radio mass-loss rate is found to be $5.74 \pm 0.71 \times 10^{-5} M_{\odot} \mathrm{yr}^{-1}$, with the mm mass-loss rate previously measured as $3.57 \times 10^{-5} M_{\odot} \mathrm{yr}^{-1}$ (F18).

A lower value in the radio than in the millimetre was found for WR I. The mass-loss rate determined in the radio is $3.10 \times 10^{-5} M_{\odot} \mathrm{yr}^{-1}$, in comparison to the $\dot{M} f_{\mathrm{cl}}^{\frac{1}{2}}$ value calculated from the millimetre of $3.55 \times 10^{-5} M_{\odot} \mathrm{yr}^{-1}$ (F18), however due to the uncertainties on the radio $\dot{M} f_{\text {cl }}$, the two values could still potentially be consistent. Higher values are also found in the millimetre for $\dot{M} f_{\mathrm{cl}}^{\frac{1}{2}}$ for WR E. The $\dot{M} f_{\mathrm{cl}}^{\frac{1}{2}}$ value from the radio observations is calculated to be $3.62 \pm 0.42 \times 10^{-5} M_{\odot} \mathrm{yr}^{-1}$ for WR E, which can be compared to the mm mass-loss rate from the millimetre, of $5.24 \times 10^{-5}$ (F18).

We can also compare our mass-loss rates to the previous radio results in D10. In D10, mass-loss rates were found for two of the WR stars they detect - WR L and WR F. For WR L, they find a mass-loss rate of $2 \times 10^{-5}\left(v_{\infty} / 1000 \mathrm{~km} \mathrm{~s}^{-1}\right) M_{\odot} \mathrm{yr}^{-1}$. By taking the terminal velocity of this source to be $v_{\infty} \sim 700 \mathrm{~km} \mathrm{~s}^{-1}$, this leads to a value of $1.4 \times 10^{-5} M_{\odot} \mathrm{yr}^{-1}$. In our observations, we determine a higher mass-loss rate of $2.72 \times 10^{-5} M_{\odot} \mathrm{yr}^{-1}$.

In D10, a mass-loss rate of $3.3 \times 10^{-5}\left(v_{\infty} / 1000 \mathrm{~km} \mathrm{~s}^{-1}\right)$ $M_{\odot} \mathrm{yr}^{-1}$ was found for WR F. With an expected $v_{\infty}$ value of $1200 \mathrm{~km} \mathrm{~s}^{-1}$, this would lead to a mass-loss rate of $3.96 \times 10^{-5} M_{\odot} \mathrm{yr}^{-1}$. Our mass-loss rate calculation of WR F results in a higher value, $6.62 \pm 0.34 \times 10^{-5} M_{\odot} \mathrm{yr}^{-1}$. We can also consider the predicted mass-loss rate derived for WR F of $\sim 3.16 \times 10^{-5} M_{\odot} \mathrm{yr}^{-1}$ from Clark et al. (2011). These values can be reconciled if there are higher levels of clumping in the inner parts of the winds, as the Clark et al. (2011) value is derived from optical and near-IR data.

Mean mass-loss rates could be determined for the different classes of WR stars, with a mean mass-loss rate over the total ensemble calculated to be $4.61 \times 10^{-5} M_{\odot} \mathrm{yr}^{-1}$. The average mass-loss rate for the $\mathrm{WN}$ classes is determined as $4.26 \times 10^{-5} M_{\odot} \mathrm{yr}^{-1}$, and for WC subclasses, it is found to be $5.33 \times 10^{-5} M_{\odot} \mathrm{yr}^{-1}$. In general, the WC subtype can be seen to have higher values of mass-loss rates on average, but there were only measurements from three WC stars, and one of these is a likely binary (WR F).

These values can be considered to be fairly consistent with previous mass-loss determinations of WR stars, with a value from Leitherer et al. (1997), of $\dot{M} \sim 4 \times 10^{-5} M_{\odot} \mathrm{yr}^{-1}$ over all spectral sub-types. Measurements from Cappa et al. (2004) gave $\dot{M} \sim 4 \pm 3 \times 10^{-5} M_{\odot} \mathrm{yr}^{-1}$ for $\mathrm{WN}$, so our WN mean value can be seen to be consistent, but their measurement for the WC subtype was $\sim 2 \pm 1 \times 10^{-5} M_{\odot} \mathrm{yr}^{-1}$, lower than our average value found for this spectral subtype. The overall spread of the mass-loss rates is found to be similar to that seen in $\mathrm{F} 18$, from 1.52 to $9.75 \times 10^{-5} M_{\odot} \mathrm{yr}^{-1}$ (ignoring the WN10-11h/BHG star, WR S).

\subsubsection{Clumping ratios}

Due to the different wavelength regimes utilised for the calculating $\dot{M} f_{\mathrm{cl}}^{\frac{1}{2}}$, then different clumping factors may play a role in the value of $\dot{M} f_{\text {cl }}^{\frac{1}{2}}$ calculated, assuming a constant mass-loss rate throughout the wind. Unfortunately, the intrinsic mass-loss rate for these winds is unknown, so we can only consider the relative degrees of clumping for different wavelength regimes. This still gives an indication of the different levels of clumping present at varying radial geometries within the stellar winds.

Clumping ratios were determined by comparing stellar massloss rates at different frequencies. The datasets which contained tapered $u-v$ visibilities were used, to allow for the comparison between the millimetre and radio observations as previously discussed. Clumping ratios were derived for seven WR stars from the tapered datasets, shown in Table 7. These ratios were calculated assuming a normalisation of the mass-loss rate (using $\dot{M} f_{\mathrm{cl}}^{\frac{1}{2}}$ values from TAPERALMA) and clumping ratios were derived separately for the TAPER5 and TAPER 9 mass-loss rates against this normalised value.

A large spread of clumping ratios are found over the different Wolf-Rayet stars. The majority of the WR stars (WR A, G, L, F, and $\mathrm{M}$ ) demonstrate the expected behaviour of the clumping factor, decreasing as the wavelength increases. This is not seen for WR D, which has a clumping ratio of 1.34 , significantly above unity. This could indicate that the wind is highly clumped even out at radio wavelengths, in the most extended part of the wind. The adverse clumping ratio (above the expected fraction for an outer part of the wind) could be related to the fact that WR D is a binary candidate. As the calculated mass-loss rate is reliant on assuming fluxes are dominated by thermal emission from stellar winds of a single star, non-thermal radio components due to binarity could have led to these unexpected results.

These sources don't give a clear indication of a uniform trend for clumping ratios between the $\mathrm{mm}$ and the radio-photosphere. In general, the results are found to support the belief that in stellar winds, clumping should decrease within the wind as a function of radial distance from the star. 
Table 7. Clumping ratios of WRs.

\begin{tabular}{|c|c|c|c|c|c|c|}
\hline \multirow[t]{2}{*}{ Source } & \multirow[t]{2}{*}{ Spectral type } & \multicolumn{2}{|c|}{ ATCA $\dot{M} f_{\mathrm{cl}}^{\frac{1}{2}}\left(M_{\odot} \mathrm{yr}^{-1}\right)$} & \multirow[t]{2}{*}{$\mathrm{ALMA} \dot{M} f_{\mathrm{cl}}^{\frac{1}{2}}\left(M_{\odot} \mathrm{yr}^{-1}\right)$} & \multirow[t]{2}{*}{$\frac{f_{\mathrm{cl}, 5.5}}{f_{\mathrm{cl}, \mathrm{ALMA}}}$} & \multirow{2}{*}{$\frac{f_{\mathrm{cl}, 9}}{f_{\mathrm{cl}, \mathrm{ALMA}}}$} \\
\hline & & $5.5 \mathrm{GHz}$ & $9 \mathrm{GHz}$ & & & \\
\hline WR A & $\mathrm{WN} 7 \mathrm{~b}+\mathrm{OB} ?$ & $6.06 \pm 0.59 \mathrm{e}-05$ & $6.63 \pm 0.26 e-05$ & $6.63 \pm 0.14 \mathrm{e}-05$ & $0.84 \pm 0.21$ & $1.00 \pm 0.09$ \\
\hline WR D & WN7o & - & $1.84 \pm 0.41 \mathrm{e}-05$ & $1.59 \pm 0.12 \mathrm{e}-05$ & - & $1.34 \pm 0.63$ \\
\hline WR G & WN7o & - & $1.50 \pm 0.35 e-05$ & $1.87 \pm 0.14 \mathrm{e}-05$ & - & $0.64 \pm 0.31$ \\
\hline WR I & WN8o & $1.70 \pm 0.32 \mathrm{e}-05$ & $3.06 \pm 0.20 \mathrm{e}-05$ & $3.15 \pm 0.05 \mathrm{e}-05$ & $0.29 \pm 0.11$ & $1.47 \pm 0.19$ \\
\hline WR L & WN9h+OB? & $1.66 \pm 0.31 \mathrm{e}-05$ & $2.67 \pm 0.16 e-05$ & $3.36 \pm 0.05 \mathrm{e}-05$ & $0.24 \pm 0.09$ & $0.63 \pm 0.08$ \\
\hline WR F & $\mathrm{WC} 9 \mathrm{~d}+\mathrm{OB} ?$ & - & $3.07 \pm 0.25 \mathrm{e}-05$ & $6.24 \pm 0.22 \mathrm{e}-05$ & - & $0.24 \pm 0.04$ \\
\hline WR M & WC9d & - & $1.23 \pm 0.25 \mathrm{e}-05$ & $2.59 \pm 0.25 \mathrm{e}-05$ & - & $0.23 \pm 0.10$ \\
\hline
\end{tabular}

Notes. Clumping ratios were derived from dividing calculated values of $\dot{M} f_{\mathrm{cl}}^{\frac{1}{2}}$ from fluxes measured with the TAPER5 and TAPER9 datasets, against values found for $\dot{M} f_{\mathrm{cl}}^{\frac{1}{2}}$ from fluxes given by TAPERALMA. In order to calculate clumping ratios from different frequencies of emission, a constant mass-loss rate is assumed throughout the wind (normalised to the $\dot{M} f_{\mathrm{cl}}^{\frac{1}{2}}$ values given by TAPERALMA).

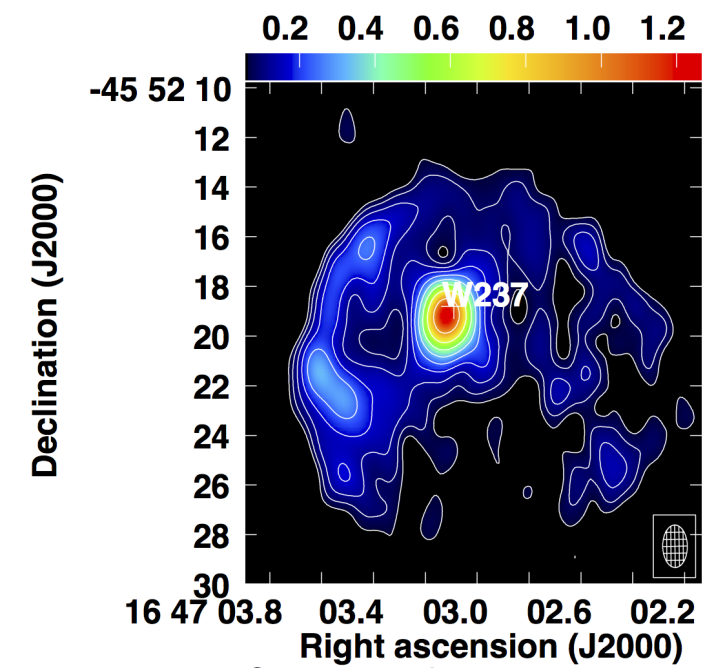

Fig. 7. Non-primary beam corrected image of RSG W237 from FULLCONCAT dataset. Contours are plotted at 1, 1.4, 2, 2.8, 4, 5.7, 8, $11.3,16,22.6,32,45.25,64,90.50 \times \sigma$, the local rms value.

\subsection{Cool supergiants and hypergiants}

Nine of the ten RSGs and YHGs present in Westerlund 1 are detected, with the singular exception of the YHG W8a. Eight of these sources are resolved, with extended nebulae detected around all of these sources. As these are all cool supergiants and hypergiants, the typical photospheric temperatures of $\sim 4000 \mathrm{~K}$ for RSGs and 4000-8000 K for YHGs would not be enough to cause the ionisation of the nebulae material and lead to radio emission. Instead, a diffuse radiation field caused by the hundreds of $\mathrm{O}$ and $\mathrm{B}$ stars present in the cluster was postulated as the source of the ionisation, allowing for the stellar nebulae to be observed.

The structures of the extended emission around these sources are seen to be asymmetric and complex in nature. A mixture of morphologies were seen around several of the sources, including the bow shock structure as seen around W237 in Fig. 7 and the cometary morphology around W265, as shown in Fig. 9.

Spectral indices are determined for all detected RSGs and YHGs except the source W32. A marginal detection could be found from the TAPER9 dataset at $\sim 0.13 \mathrm{mJy}$, (with a S/N of only $\sim 2.69$ ), alongside a marginal detection of $\sim 0.08 \mathrm{mJy}$ in the TAPER5 dataset (at a S/N of 1.26). The ALMA flux of W32

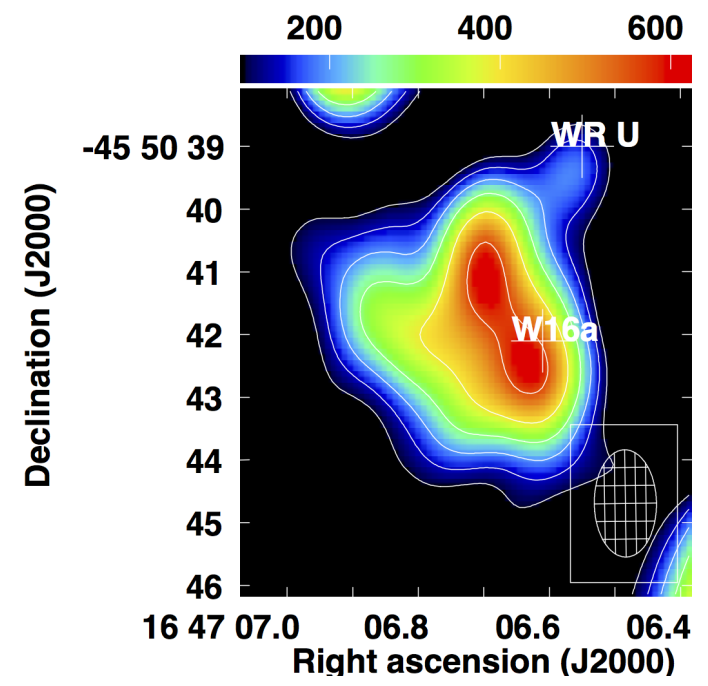

Fig. 8. Non-primary beam corrected image of YHG W16a from FULlCONCAT dataset. Contours are plotted at 1, 1.4, 2, 2.8, 4, 5.7, 8, $11.3,16,22.6,32,45.25,64,90.50 \times \sigma$, the local rms value.

from F18, $0.38 \pm 0.07 \mathrm{mJy}$, in combination with marginal detections suggest that the emission is of a thermal nature.

Core components of the source emission are used to calculate spectral indices. For most of the cool supergiants and hypergiants, spectral indices are found to be extremely flat, such as $0.00 \pm 0.04$ for $\mathrm{W} 237,-0.01 \pm 0.12$ for $\mathrm{W} 75$, and $-0.01 \pm 0.04$ for W4a, consistent with composite emission of both optically thin and thick components. This was seen previously in the millimetre (F18). Amongst the YHGs, some sources are found to have more negative spectral indices, including W265 and W12a, which have spectral indices of $-0.13 \pm 0.23$ and $-0.30 \pm 0.07$ respectively. Due to the relatively high uncertainty on these values, this is consistent with the presence of non-thermal emission, but also consistent with optically thin emission surrounding these sources.

W16a, shown in Fig. 8, is found to have a spectral index $-0.10 \pm 0.05$, which would suggest the presence of non-thermal or optically thin emission components. This object may actually have a much flatter spectral index, as there is possible source confusion due to extremely close presence of WR U, which is not readily distinguished from the emission associated with W16a. 


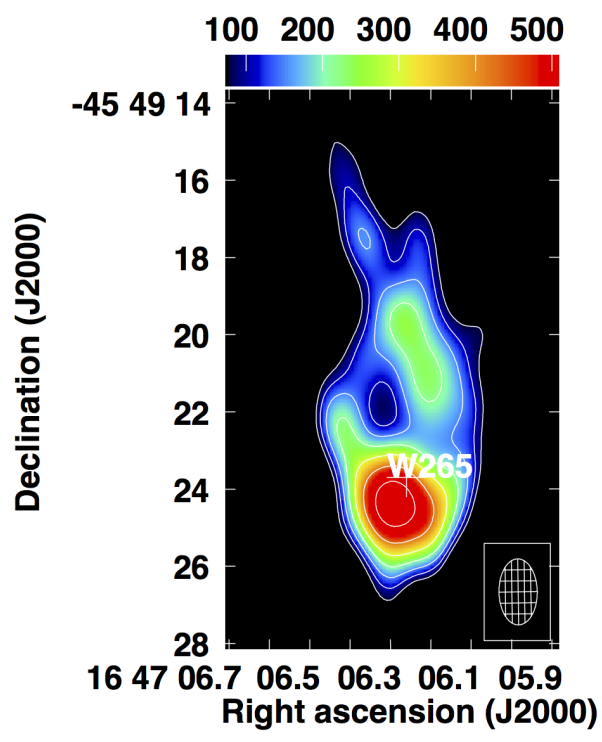

Fig. 9. Non-primary beam corrected image of YHG W265 from FULLCONCAT dataset. Contours are plotted at 1, 1.4, 2, 2.8, 4, 5.7, 8, $11.3,16,22.6,32,45.25,64,90.50 \times \sigma$, the local rms value.

Table 8. Mass-loss estimates for YHGs and RSGs.

\begin{tabular}{lll}
\hline Source & Spectral type & ATCA $\dot{M} f_{\mathrm{cl}}^{\frac{1}{2}}\left(M_{\odot} \mathrm{yr}^{-1}\right)$ \\
\hline W12a & F1Ia $^{+}$ & $1.66 \pm 0.02 \times 10^{-5}$ \\
W4a & F3Ia $^{+}$ & $2.59 \pm 0.02 \times 10^{-5}$ \\
W32 & F5Ia $^{+}$ & $3.01 \pm 0.50 \times 10^{-6}$ \\
W265 & F5Ia $^{+}$ & $1.10 \pm 0.03 \times 10^{-5}$ \\
W237 & M3Ia & $2.00 \pm 0.02 \times 10^{-6}$ \\
W75 & M4Ia & $7.27 \pm 0.48 \times 10^{-7}$ \\
W20 & M5Ia & $3.28 \pm 0.03 \times 10^{-6}$ \\
\hline
\end{tabular}

Notes. Fluxes were used from core components of the emission attributed to the YHGs and RSGs, as given by the FULLCONCAT dataset. These mass-loss rates are calculated for objects where an approximation of a thermally emitting stellar wind is considered valid, using the Wright and Barlow equation (Wright \& Barlow 1975).

Mass-loss rates were calculated for sources for which the emission present could be attributed to optically thin and thick components in the stellar wind, excluding sources with potential non-thermal emission. The core component of flux detected for these stellar sources were used in the mass-loss rate calculation, with the exception of W75, which was found to be far fainter than the rest of the sources. W26 and W16a were not considered as no core component could be isolated within from the emission associated with these sources. The mass-loss rate estimates are presented in Table 8 .

We can compare the range of mass-loss rates found for RSGs, $\sim 10^{-7}-10^{-6} M_{\odot} \mathrm{yr}^{-1}$, to that found for field RSGs, which vary from $10^{-6}$ to $10^{-4} M_{\odot} \mathrm{yr}^{-1}$ (Jura \& Kleinmann 1990; Sylvester et al. 1998). A RSG with similar morphology, VY CMa, has been found to have mass-loss values of $\dot{M} f_{\text {cl }}^{\frac{1}{2}} \sim 10^{-3} M_{\odot} \mathrm{yr}^{-1}$ or greater (Shenoy et al. 2016). For W237, a time-averaged mass-loss rate was previously calculated in F18, $\sim 10^{-5} M_{\odot} \mathrm{yr}^{-1}$, which is significantly higher than the value determined here, but uses the measure of the ionised mass of the extended nebula surrounding the source.
There is a clear caveat in comparing the mass-loss rate estimates determined here to those calculated in the literature. The methods used to determine the $\dot{M} f_{\text {cl }}^{\frac{1}{2}}$ values here are derived from the free-free emission in the wind, whereas $\dot{M} f_{\mathrm{cl}}^{\frac{1}{2}}$ values in the literature are typically calculated from fitting models to the metal lines of stars, as seen for YHGs such as $\rho$ Cas, (Lobel et al. 1998, 2003), or from the modelling of low excitation CO lines and using models to extrapolate the mass-loss rates, as has been carried out for the YHG IRC+10420 (Oudmaijer et al. 1996; Castro-Carrizo et al. 2007), and the RSG VY CMa (Decin et al. 2006). These models often require many assumptions to be made about the velocity and temperature structure of the stellar wind, as well as the heating and cooling processes occurring within the wind, and the relative gas and dust densities. These methods also use information from the emission of different wavelength regimes, and so the effect of clumping and structure within the wind could lead to inconsistent results between our estimates and the literature.

The range of $\dot{M} f_{\mathrm{cl}}^{\frac{1}{2}} \sim 10^{-6}-10^{-5} M_{\odot} \mathrm{yr}^{-1}$ is found for the YHGs present in Westerlund 1. Some direct comparisons can be made to previous observations, including $\mathrm{W} 4 \mathrm{a}$, where a massloss rate estimate was inferred from millimetre observations, $\sim 10^{-5} M_{\odot} \mathrm{yr}^{-1}$ (F18). This is found to be consistent with our estimate. D10 suggest an estimate of $\sim 10^{-5} M_{\odot} \mathrm{yr}^{-1}$ for W265. Our mass-loss rate for this source, $1.10 \pm 0.03 \times 10^{-5} M_{\odot} \mathrm{yr}^{-1}$, is therefore also consistent with this earlier estimate, even though the D10 mass-loss rate was derived from more the extended region of emission.

\section{3. $O B$ supergiants, hypergiants, $L B V s$, and $s g B[e]$ stars}

Of the radio detections made from the survey of Westerlund 1, 11 of these are OBA stars. This includes the LBV A2 Ia star W243, the $\operatorname{sgB}[\mathrm{e}]$ star W9, seven confirmed OB supergiants, and two radio sources previously discovered in D10, D09-R1 and D09$\mathrm{R} 2$, which have the assigned spectral type of blue supergiants (BSGs). The detections cover a range of spectral types, from late type O supergiants (O9Ib) to early type B supergiants (B1Ia). No stars from a later spectral type were detected. This indicates that the majority of stars within the cluster exhibit typical thermal spectral behaviour, and so their expected radio fluxes fall well below the noise level for these observations (as was indicated by the lack of detections for most OB stars in the cluster from previous millimetre observations; Fenech et al. 2018).

\subsubsection{OB super and hypergiants}

In total, nine OB stars are detected in the cluster with five new radio detections. The star W10 is a known double-lined spectroscopic binary (SB2) binary in the cluster, with stellar components $\mathrm{B} 0.5 \mathrm{I}+\mathrm{OB}$, and is found to have an associated flux density of $0.16 \pm 0.05 \mathrm{mJy}$. Two early type B supergiants, W18 and W19, with spectral types B0.5Ia and B1Ia, are found to have fluxes $0.36 \pm 0.06 \mathrm{mJy}$ and $0.08 \pm 0.03 \mathrm{mJy}$ respectively. Additionally, two late type $\mathrm{O}$ supergiants are newly detected, W1031 of spectral type O9III with a flux density of $0.90 \pm 0.05 \mathrm{mJy}$, and W1056, a O9.5II star, with a flux density measured of $0.09 \pm 0.02 \mathrm{mJy}$.

Cluster members D09-R1 and D09-R2, which have the current spectral type designation of BSG, were previously discovered in the radio (D10). These sources currently lack an accurate spectral classification. Fluxes are found for D09-R1 
for both a core component and total value of $1.29 \pm 0.03 \mathrm{mJy}$ and $5.44 \pm 0.10 \mathrm{mJy}$. D09-R1 is embedded in an area of diffuse radio emission, as was previously indicated in D10. A flux is also determined for the source D09-R2, and found to be $0.96 \pm 0.06 \mathrm{mJy}$.

As these sources were also detected in the millimetre, spectral indices could be determined. They were found to have fairly flat, slightly negative spectral indices of $\sim-0.01$ and -0.03 , for D09-R1 and D09-R2 respectively. This suggests that these objects experience a composite behaviour. This could include a combination of partially optically thick, optically thin and nonthermal emission components. The spectral indices are roughly consistent with previous radio-mm spectral indices, with the spectral index determined for D09-R1 slightly flatter in comparison to a previous radio-mm measurement of $\sim 0.17$ (F18).

Two OB stellar sources, W17 and W15, both had previous radio detections (D10). W15 is currently assigned the spectral classification $\mathrm{O} 9 \mathrm{Ib}$, and is found to have a flux density of $1.65 \pm 0.09 \mathrm{mJy}$. The late $\mathrm{O}$ supergiant $\mathrm{W} 17$ is found to have a radio flux of $0.98 \pm 0.04 \mathrm{mJy}$ for the core component of the source, surrounded by an extended component with a total flux density of $2.04 \pm 0.08 \mathrm{mJy}$. This star was also detected in the millimetre, and so a spectral index was derived for this source of $\sim-0.03$, suggesting a composite spectra, which may be made up of optically thin and thick, as well as non-thermal components. This revised spectral index was found to be significantly flattened in comparison to the previous radio-mm spectral index found of $\sim 0.33$ (F18).

As the emission from W17 could be attributed to optically thick and thin components, an approximation could be made to a thermal stellar wind in order to determine the massloss rate. A mass-loss rate was determined for this source of $1.38 \pm 0.03 \times 10^{-4} M_{\odot} \mathrm{yr}^{-1}$. This is higher than would be expected for a late $\mathrm{O}$ supergiant. This suggests that the approximation of a thermally emitting stellar wind is not suitable, and may indicate the presence of binarity, supported by the fact that W17 has already been found to exhibit variability (Bonanos 2007).

Six of the OB supergiants did not have counterpart millimetre detections. This included a range of spectral types, over B1Ia - O9Ib. The only one of these sources previously detected in the radio in D10 was W15.

By using the flux limits of the ALMA dataset presented in F18, spectral index limits could be determined. These limits involved the use of the FULLCONCAT dataset, rather than the tapered datasets, due to the intrinsic faintness of the sources. All of the stars were found to have spectral index limits consistent with non-thermal emission, with especially strongly negative spectral indices for W15 and W1031. We considered the approximation of the radio fluxes to thermal stellar wind emission, to determine mass-loss rate estimates. The range of massloss rates found for the OB supergiants varied from $4.8 \times 10^{-6}$ to $1.31 \times 10^{-4} M_{\odot} \mathrm{yr}^{-1}$.

Although the lower end of this range is in line with expectations, in general these values are much higher than would be expected for late $\mathrm{O}$ and early $\mathrm{B}$ type supergiants, $\sim 10^{-6}-$ $10^{-7} M_{\odot} \mathrm{yr}^{-1}$. The high mass-loss rates, along with the negative spectral index limits derived, indicate that attributing the radio emission of these stars to stellar winds from single stars is not valid. It is then worth considering that these stars may be binaries.

There was no prior evidence from hard X-ray detections or radial velocity (RV) variability for $\mathrm{W} 15$, but a clear constraint was found from its spectral index limit of $<-1.15$, strongly suggesting non-thermal emission. If W15 is a binary system, then this system must be either in an eccentric or a very wide orbit in order to be consistent with other observational results for this star. A wide system would allow for the WCZ to occur outside of the radio photosphere, so non-thermal emission would not be obscured by surrounding thermal emission from a stellar wind, and the proximity between the WCZ and the star would be insufficient for shocks to produce hard X-rays, as would typically be expected for binaries (Pittard \& Dawson 2018). This is also supported by recent observational evidence that binary stars have been seen to present X-ray signatures that are consistent with single stars of a similar spectral type (Clark et al. 2019c, and references within).

This system can be compared to the WR star, WR 140. The source WR 140 is considered to be the archetypal wind colliding binary (WCB), consisting of a WC7 WR and an O5 companion. WR 140 has been found to have variable emission due to the high eccentricity of the orbit ( 0.90, Fahed et al. 2011), with different variabilities measured at various radio wavelengths (Dougherty et al. 2011). WR 140 has been detected to have X-ray variability, including in the hardness of its X-ray spectra over the orbital phase (Corcoran et al. 2011). If W15 is a similar system, then this scenario could explain the lack of a hard X-ray detection.

Another piece of evidence supporting this hypothesis is the variability seen in the radio flux between the D10 8.6 GHz and ATCA $9 \mathrm{GHz}$ observations - where the increase in flux is almost a factor of two. This variance could be due to a change in orbital phase between the two measurements. A further time-domain study of W15 would allow for the potential to map out possible changes of orientation over orbital phase, in order to provide constraints on the properties of the system.

The only previously confirmed binary of the detected OB supergiants was W10, first presented as an SB2 in Clark et al. (2008). W10 has also been previously found to be an RV variable. W10 was recently found to experience softer and dimmer X-ray emission than other SB2s in Westerlund 1, suggesting an eccentric binary where the emission from a WCZ was less present in observations taken in 2005 (Clark et al. 2019c). This may be supported by the weak constraint on the nature of emission for W10, with a spectral index limit of $<-0.04$, consistent with a composite spectra.

$\mathrm{W} 18$ is found to have a negative spectral index limit, $<-0.43$, strongly indicative of non-thermal emission. This stellar source has no current associated evidence for binarity from X-ray observations and no RV detections. This doesn't rule out binarity for this source, as bias in the observational limits of these methods could mean that a binary system is present that was not easily detected by RV variations. The surrounding dense cluster wind and material from other stars in the cluster could also obscure $\mathrm{X}$-ray emission, as there is diffuse $\mathrm{X}$-ray emission known to be present throughout the cluster (Muno et al. 2006). W19 is also a cluster member with detected radio emission but no prior evidence of binarity from RV or X-ray measurements, although it has previously been found to be $\mathrm{H} \alpha$ variable. It has the weakest constraint on its spectral index limit, allowing for a flat composite spectrum of emission. It is also of note that W18, W19, W10, and W15, have all been previously found to be associated with long period or aperiodic variability, supportive of the conclusion of binarity (Bonanos 2007).

The other two stellar cluster members detected, W1031 and W1056, are both confirmed RV variables. The period for W1031 is not currently determined. W1056 is known to have a candidate period of less than ten days. It's of note that W1056 has a much looser constraint on the spectral index, $<0.02$, in comparison to 
the other stars with late $\mathrm{O}$ spectral classification, which may be consistent with a flat composite spectra, which would be expected if stellar winds are obscuring some of the radio emission from the colliding wind region.

We suggest that we would not expect to detect any emission from purely thermal stellar wind emitters in the radio. This is supported by the lack of millimetre detections in F18 for the majority of OB supergiants in Westerlund 1. With this in mind, along with the previously observed binarity characteristics for many of these objects in the UV and X-ray, we can infer that all of the OB supergiants we detect are either binaries or binary candidates. We believe most of these objects are highly likely to be long period binaries, where the separation between the stellar components is sufficiently high for non-thermal emission from colliding wind regions to occur outside the region of the stellar wind radio photospheres. Therefore, we have managed to detect three new binary candidates, that are likely long period binaries where RV variations could not be detected.

\subsubsection{W243}

W243, a LBV with assigned spectral type A2 Ia, is detected in the ATCA observations. The spectral index is found to be $\sim 0.82$, consistent with previous measurements. This is indicative of a thermal spectrum, with a slight steepening in comparison to the canonical stellar wind value. Our mass-loss rate determination for this object is found to be $3.12 \times 10^{-5} M_{\odot} \mathrm{yr}^{-1}$.

A mass-loss estimate was previously determined for W1243 in $\mathrm{D} 10$, on the order of $1.1 \times 10^{-5}\left(v_{\infty} / 200 \mathrm{~km} \mathrm{~s}^{-1}\right) M_{\odot} \mathrm{yr}^{-1}$. From an assumed $v_{\infty} \sim 550 \mathrm{~km} \mathrm{~s}^{-1}$ for W243, the mass-loss rates therefore can be considered to be consistent. We can also compare this mass-loss rate to the previous millimetre observations, which gave a $\dot{M}$ of $\sim 2.5 \times 10^{-5} M_{\odot} \mathrm{yr}^{-1}$ (F18). The radio and $\mathrm{mm}$ values are both higher than the expected mass-loss rate, $\dot{M} \sim 8 \times 10^{-7} M_{\odot} \mathrm{yr}^{-1}$, given from modelling in Ritchie et al. (2009). Clear limitations for this modelling included the issue of fitting to all $\mathrm{H}$ and $\mathrm{He}$ emission features prominent in the spectra, suggesting that the spectral type approximation used was not suitable in order to generate an accurate fit.

This LBV $\dot{M} f_{\mathrm{cl}}^{\frac{1}{2}}$ value is also larger than a mass-loss rate of $\sim 1.35 \times 10^{-5} M_{\odot} \mathrm{yr}^{-1}$, found from radio observations of a postRSG LBV HD160529 (Leitherer et al. 1995). It can also be compared to other LBV measurements from sources including AFGL2338, AG Car, FMM362, and the Pistol Star (Clark et al. 2009; Najarro et al. 2009; Groh et al. 2009), which have massloss rates over the range of $3-6 \times 10^{-5} M_{\odot} \mathrm{yr}^{-1}$, all larger than the mass-loss rate calculated for W243.

An estimate of the clumping ratios are also found for this object, between the millimetre and radio data. The clumping ratios are found to be $\sim 0.027$ and $\sim 0.07$ when comparing $5.5 \mathrm{GHz}$ and $9 \mathrm{GHz}$ respectively to the millimetre observations, suggesting a much higher level of structure and clumping within the inner regions of the wind sampled by the $\mathrm{mm}$ observations, that then decreases with increasing distance from the star.

\subsubsection{W9}

W9 can be seen in Fig. 10, where the two filaments of emission to the south-east and south-west of the source as observed previously in millimetre observations (Fenech et al. 2017; F18) can be seen at much lower resolution in the radio, as well as an additional third filament to the north-west. The flux of the core compact source region of W9 is found to be $27.38 \pm 0.06 \mathrm{mJy}$,

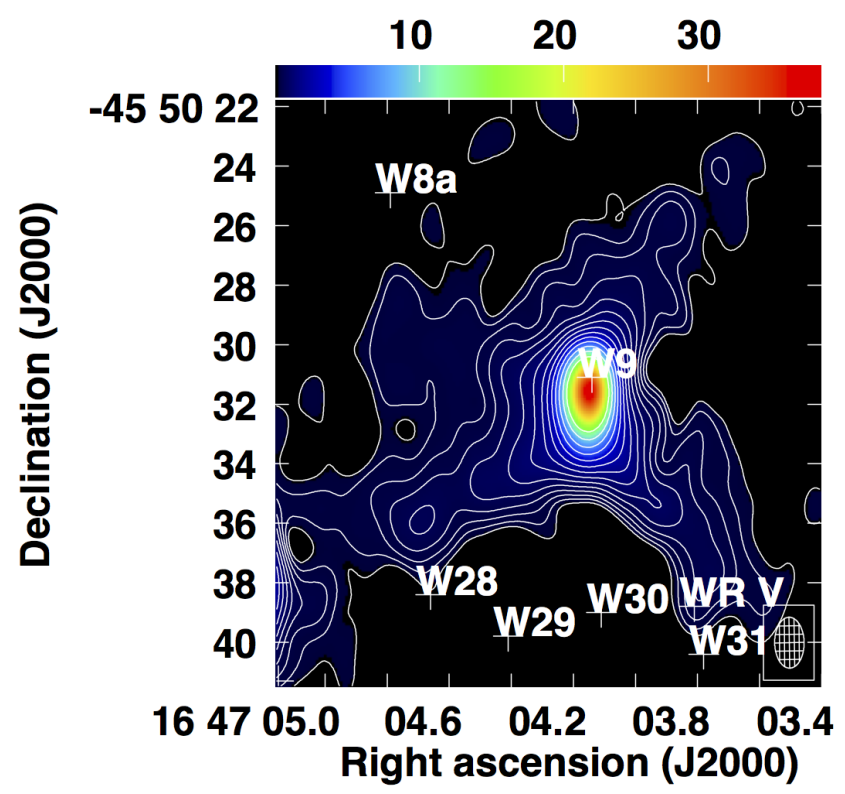

Fig. 10. Non-primary beam corrected image of stellar source sgB[e] W9 from FULLCONCAT dataset. Contours are plotted at 1, 1.4, 2, 2.8, 4, 5.7, $8,11.3,16,22.6,32,45.25,64,90.50 \times 150 \mu \mathrm{Jy}$.

and the extended region around it gives a total flux value of $83.14 \pm 0.41 \mathrm{mJy}$. The spectral index of W9 indicates thermal emission, in line with the canonical value for a stellar wind, with a value of $\alpha=0.68 \pm 0.02$. This value is consistent with the previous measurements.

As W9 was found to have a thermal nature derived from the spectral index, we could then make an estimate of the approximate mass-loss rate for this source, despite the evidence that this source is known to be a binary from its X-ray emission (Clark et al. 2013). The mass-loss rate is calculated to be $\dot{M} f_{\text {cl }}^{\frac{1}{2}} \sim 13.03 \pm 0.01 \times 10^{-5} M_{\odot} \mathrm{yr}^{-1}$, similar to the value found from Model 2 of W9 in D10.

\section{Origin of the extended emission within the cluster}

As was previously introduced in Sect. 4, there is a pervasive level of diffuse radio emission that can be seen throughout the cluster. This can be seen most clearly by looking at Fig. 1, which shows the ATCA observations separated by configuration. The spatial scales measured by the shortest configuration with a maximum baseline of $750 \mathrm{~m}$ demonstrate a significant amount of diffuse radio emission present throughout the cluster. Much of this extended radio emission appears to be centred on the radio bright sources, such as W9, W20, or even the WR star WR B, but some of the extended emission cannot be attributed to any of the known stellar sources. These areas still contain compact sources with no stellar counterpart, as discussed in Sect. 5.2. These areas of concentrated emission that appear throughout the cluster indicate the high level of varying structure present, and that the overall structure of the diffuse emission is clumped and non-spherical.

\subsection{Stellar and cluster wind interaction}

From the morphology of this diffuse background, as seen clearly in all the figures displaying the whole cluster region (Figs. 13 , B.1), we can see this diffuse emission is more concentrated 
towards the centre of the cluster. At extended distances from stellar sources, a large level of radio emission is detected, indicating interactions between the stellar winds of the sources and a cluster wind. The interaction of stellar winds with the surrounding environment has previously been discussed in Mackey et al. (2015) as an origin of the sculpted nebulae surrounding W26. Typically, this diffuse emission would be expected to arise as a result of interaction between young massive stars and remnants of their parent giant molecular cloud (GMC), but at an expected age of $5 \mathrm{Myr}$, this is not believed to be the case for $\mathrm{Wd} 1$.

This is also supported by the general lack of evidence to support any ongoing star formation within the cluster. This is currently supported by results from many mid- and near-infrared surveys (2MASS, GLIMPSE, MIPSGAL) that have included the cluster within their field of view (Clark et al. 2015), as well as a lack of any detections of massive young stellar objects (YSOs) in the vicinity of the cluster, or the presence of any methanol masers, a typical signature of star forming regions (SFRs). We can also compare the general geometry of the diffuse emission to stellar clusters that are known to be located in star forming regions, such as the clusters Danks 1 and 2, located in G305. The structure of the diffuse emission around these clusters are seen to follow a much simpler classical wind blown shell geometry (Clark \& Porter 2004; Davies et al. 2012).

Some future observations that could help us to constrain the origin of the gas within this cluster would be to search more explicitly for spectral signatures that constrain the origin to natal emission (with linked evidence of ongoing SFRs present), or from the outflows of the stellar population. A signature of recent star formation (and hence, diffuse emission from the natal remnants) already mentioned is that of methanol masers. Future studies of transition frequencies, such as $12.2 \mathrm{GHz}$ for methanol, or $1.7 \mathrm{GHz}$ for $\mathrm{OH}$ masers, would provide evidence of star formation and hence constrain the origin of the gas; or a lack of detection would help suggest that the current conclusion of stellar emission is more valid.

A more explicit method to constrain the nature of the gas due to stellar emission would be to search for $\mathrm{He}$ and $\mathrm{N}$ emission lines, using optical and near-IR spectroscopy. For some cluster members, [N II] emission lines have previously been detected, including the RSG population (Clark et al. 2010). These emission lines are known to occur in the ejecta of other cool evolved stars with nearby stellar companions, where the external ionisation of the ejecta is due to material from the stellar companion's wind, as in the case of the YHG HR 8752 (Stickland \& Harmer 1978). Observational evidence of these emission lines within the diffuse regions of the gas would therefore provide further evidence that the extended emission can be related to stellar winds of massive stars in the cluster.

\subsection{Investigating the intra-cluster wind}

Previous evidence of a cluster wind in $\mathrm{Wd} 1$ has been found in the X-ray (Muno et al. 2006), radio (D10), and millimetre (F18). This cluster wind is also indicated by the common orientation of several of the resolved extended nebulae around the cool super and hypergiants in the cluster, where bow shocks and cometary tails are indicative of a general outward direction, originating from the centre of the cluster (Andrews et al. 2018). The origins of cluster winds are believed to be related to the feedback of stellar winds that become thermalised and then drive the cluster winds. The properties of the mechanisms of cluster wind propagation are not fully determined, due to uncertainties in the conversion of stellar kinetic energy to thermal energy
(Chevalier \& Clegg 1985; Cantó et al. 2000; Stevens \& Hartwell 2003). The origin of the possible cluster wind in Westerlund 1 is not confirmed, but is believed to be due to a diffuse radiation field arising in the cluster from the radiation pressure of the hundreds of OB stars present.

The overall flux of the cluster wind can be considered. A value of the total flux in the cluster was determined for the central $1.5^{\prime}$ of the cluster (as carried out in D10). This led to a value of the total flux present of $635 \mathrm{mJy}$ at $9 \mathrm{GHz}$ and $640 \mathrm{mJy}$ at $5.5 \mathrm{GHz}$. This was found to be significantly larger than the flux determined by D10 at $8.6 \mathrm{GHz}$ of $422 \mathrm{mJy}$ and $461 \mathrm{mJy}$ at $4.8 \mathrm{GHz}$, as well as the single dish measurements of the flux of the cluster from Kothes \& Dougherty (2007), of $450 \mathrm{mJy}$ and $499 \mathrm{mJy}$ at 4.8 and $8.6 \mathrm{GHz}$ respectively. From a total flux in the FULLCONCAT dataset of $647 \mathrm{mJy}$, this leads a to flux for the extended emission (with the stellar components subtracted by considering flux from known detections), of $408 \mathrm{mJy}$ over the FULLCONCAT dataset. A general estimate of ionised mass present in the cluster can be considered from $\sim 408 \mathrm{mJy}$ flux found for the extended emission at $9 \mathrm{GHz}$ over a assumed spherical region of diameter $1.5 \mathrm{arcmin}$, gave a value of $29 M_{\odot}$, (assuming a general plasma temperature of $10 \mathrm{kK}$ ), almost twice the ionised mass previously determined for the extended emission in the cluster (Kothes \& Dougherty 2007; Dougherty et al. 2010).

By characterising the cluster wind, we can hope to learn more about the stellar ejecta, and how it is impacted, not only by the physics of stellar outflows, but also by the differing surrounding environments of these stars. These may play a role in sculpting and shaping the ejecta, as is clearly seen here. The presence of this cluster wind indicates the important role that an ionisation field may play on stellar populations within large clusters and associations.

Understanding the diffuse emission and its origin is important not only for determining accurately the level of material that is associated with each stellar object, but also in starting to consider how the stellar ejecta are affected by a cluster wind. Characterising the cluster wind and the interaction between an intra-cluster medium and stellar winds is important for many areas of astrophysics. The processes involved have an impact on the complete life-cycles of stars, from start to end. Cluster winds may play a role in both inhibiting and triggering the process of star formation, and may also lead to the disruption of natal giant molecular clouds. It also helps in formalising the feedback from stars into their surrounding environment, understanding resultant galactic superwinds as well as the production of cosmic rays, for which the production mechanisms require a significant level of interstellar material, or a strong radiation field, evidence for both of which can be seen in Westerlund 1 .

A dense background may also affect the resulting $\mathrm{SNe}$ that will occur from many stars when they reach their stellar endpoint. Interactions between stars and their surrounding circumstellar nebulae can impact the resultant SNe light curve, and so affect the relative ratios observed of each $\mathrm{SNe}$ type. This may in turn impact the expected ratios of observed SNe in the early universe. This shows a clear wider implication of this environment for a wide range of fields of study within astrophysics.

\section{Conclusion}

This paper has presented the full results of a radio census of the massive stellar cluster Westerlund 1. Observations were made using ATCA, over four different pointings of varying maximum baselines from $750 \mathrm{~m}$ to $6 \mathrm{~km}$, and over two spectral windows 
centred at $5.5 \mathrm{GHz}$ and $9 \mathrm{GHz}$. The census aimed to present a direct follow up to previous observations of the rich co-eval population of Westerlund 1, especially the previous radio ATCA observations (D10). Thirty stellar sources are detected in the radio, over a range of spectral types. A strong and diffuse radio background was also clearly seen to be present in the cluster. Five new detections are made in the radio for WR stars in the cluster, and five new radio detections are made of OB supergiants in the cluster. For sources that had previously been detected in the radio, new details of surrounding extended structures of emission have come to light, due to the better resolution and sensitivity now possible with ATCA. From the five new OB radio detections, we are able to determine the presence of three new binary candidates.

Many of the sources have been found to exhibit emission that has a spectral index fully consistent with the canonical value for thermal emission from a single stellar wind. The use of the Wright and Barlow formalism, (Wright \& Barlow 1975), allowed for mass-loss rates to be determined for a number of the sources. This was carried out for sources which were found to have spectral indices indicative of thermal emission. Clumping ratios could then be determined for many of the WR stars, with comparison between the radio observations and previously published millimetre observations (F18).

The majority of sources detected were found to be resolved. The origin of extended emission around most of these sources is not clear. One possible explanation is due to binarity, but many of sources detected with extended emission are not binary candidates. Many of the sources are also not expected to have experienced large levels of variability or pulsations, which could have been another solution to explain the presence of the extended nebulae. The majority of the sources are post-main sequence objects, RSGs, YHGs, or WRs. There is a possibility that all these stars may have experienced previous epochs of extreme mass loss, but the degree of structure surrounding sources is not seen to vary uniformly by spectral type, which indicates it may not be due to the evolutionary status of the star.

Understanding the origin of the diffuse background and the extent of its interaction with the stellar wind material is of importance. The boundary between the stellar wind material and the surrounding diffuse emission is not clear, and shows a clear need for more observations of similar environments as well as more developed models, taking into account the interaction between stellar winds of massive stars and their parent clusters. Future observations, especially of the neutral material present in the cluster, would allow for additional consideration of the intracluster material present as well as the extended nebulae of each source, allowing for updated considerations to be made of the nebulae masses and geometries around many of the stars, especially the population of the YHGs and RSGs present in $\mathrm{Wd} 1$.

Higher resolution, longer baseline, radio observations of these objects would also be of great use. The use of higher resolution observations would allow for constraints to be applied on how to distinguish between the diffuse cluster material and the outflows from the stellar sources. Such observations may allow for any possible CWB regions to finally be resolved for many of the binary candidates present in the cluster. This includes the non-thermal emitter W15 and the other OB supergiants, W10, W18, and W1031.

More tailored modelling of mass-loss rates and consideration of clumping at different radial geometries of the wind quantifiying the radial stratification taking place would also help. This would lead to better theoretical constraints on the observations of the stellar winds across a large variety of different stellar spec- tral types, as seen to populate Westerlund 1, and could be of use in studies of stellar populations in other massive stellar clusters, as well as for field stars.

Acknowledgements. This paper makes use of the following ALMA data: ADS/JAO.ALMA/2013.1.00897.S. ALMA is a partnership of ESO (representing its member states), NSF (USA) and NINS (Japan), together with NRC (Canada), NSC and ASIAA (Taiwan), and KASI (Republic of Korea), in cooperation with the Republic of Chile. The Joint ALMA Observatory is operated by ESO, AUI/NRAO and NAOJ. H. Andrews wishes to acknowledge STFC for the funding of a PhD Studentship. D. Fenech wishes to acknowledge funding from a STFC consolidated grant (ST/M001334/1).

\section{References}

Abbott, B. P., Abbott, R., Abbott, T. D., et al. 2016, Phys. Rev. Lett., 116, 061102

Abramowski, A., Acero, F., Aharonian, F., et al. 2012, A\&A, 537, A114

Aghakhanloo, M., Murphy, J. W., Smith, N., et al. 2019, ArXiv e-prints [arXiv:1901.06582]

Aharonian, F., Yang, R., \& de Oña Wilhelmi, E. 2019, Nat. Astron., 3, 561

Andrews, H., Fenech, D., Prinja, R. K., Clark, J. S., \& Hindson, L. 2018, MNRAS, 477, L55

ATNF 2019, https://wWw.narrabri.atnf.csiro.au/calibrators/ calibrator_database.html

Bednarek, W., Pabich, J., \& Sobczak, T. 2014, Nucl. Phys. B - Proc. Suppl., 256, 107

Bell, A. R. 1978, MNRAS, 182, 147

Blomme, R. 2010, in High Energy Phenomena in Massive Stars, eds. J. Martí,

P. L. Luque-Escamilla, \& J. A. Combi, ASP Conf. Ser., 422, 178

Bonanos, A. Z. 2007, AJ, 133, 2696

Cantó, J., Raga, A. C., \& Rodríguez, L. F. 2000, ApJ, 536, 896

Cappa, C., Goss, W. M., \& van der Hucht, K. A. 2004, AJ, 127, 2885

Castro-Carrizo, A., Quintana-Lacaci, G., Bujarrabal, V., Neri, R., \& Alcolea, J. 2007, A\&A, 465, 457

Cesarsky, C. J., \& Montmerle, T. 1983, Space Sci. Rev., 36, 173

Chevalier, R. A., \& Clegg, A. W. 1985, Nature, 317, 44

Clark, J. S., \& Porter, J. M. 2004, A\&A, 427, 839

Clark, J. S., Fender, R., Waters, L., et al. 1998, MNRAS, 299, L43

Clark, J. S., Negueruela, I., Crowther, P., \& Goodwin, S. 2005, A\&A, 434, 949

Clark, J. S., Muno, M. P., Negueruela, I., et al. 2008, A\&A, 477, 147

Clark, J. S., Crowther, P. A., Larionov, V. M., et al. 2009, A\&A, 507, 1555

Clark, J. S., Ritchie, B. W., \& Negueruela, I. 2010, A\&A, 514, A87

Clark, J. S., Ritchie, B. W., Negueruela, I., et al. 2011, A\&A, 531, A28

Clark, J. S., Ritchie, B. W., \& Negueruela, I. 2013, A\&A, 560, A11

Clark, J. S., Ritchie, B. W., Najarro, F., Langer, N., \& Negueruela, I. 2014, A\&A, 565, A 90

Clark, S., Negueruela, I., Ritchie, B., et al. 2015, The Messenger, 159, 30

Clark, J. S., Najarro, F., Negueruela, I., et al. 2019a, A\&A, 623, A83

Clark, J. S., Ritchie, B. W., \& Negueruela, I. 2019b, A\&A, accepted [arXiv:1908.05616]

Clark, J. S., Ritchie, B. W., \& Negueruela, I. 2019c, A\&A, 626, A59

Corcoran, M. F., Pollock, A. M. T., Hamaguchi, K., \& Russell, C. 2011, ArXiv e-prints [arXiv:1101.1422]

Crowther, P. 2019, http://www.pacrowther.staff.shef.ac.uk/WRcat/1

Crowther, P. A., Hadfield, L. J., Clark, J. S., Negueruela, I., \& Vacca, W. D. 2006, MNRAS, 372, 1407

Damineli, A., Almeida, L. A., Blum, R. D., et al. 2016, MNRAS, 463, 2653

Davies, B., Clark, J. S., Trombley, C., et al. 2012, MNRAS, 419, 1871

Decin, L., Hony, S., de Koter, A., et al. 2006, A\&A, 456, 549

Digabel, H., \& Lantuejoul, C. 1978, Iterative Algorithms, Actes du Second Symposium Europeen d'Analyse Quantitative des Microstructures en Sciences des Materiaux, Biologie et Medecine, 85

Dougherty, S., Clark, J., Negueruela, I., Johnson, T., \& Chapman, J. 2010, A\&A, 511, A58

Dougherty, S. M., Trenton, V., \& Beasley, A. J. 2011, ArXiv e-prints [arXiv:1101.0893]

Fahed, R., Moffat, A. F. J., Zorec, J., et al. 2011, MNRAS, 418, 2

Fenech, D. M., Clark, J. S., Prinja, R. K., et al. 2017, MNRAS, 464, L75

Fenech, D. M., Clark, J. S., Prinja, R. K., et al. 2018, A\&A, 617, A137

Fullerton, A. W., Massa, D. L., \& Prinja, R. K. 2006, ApJ, 637, 1025

Groh, J. H., Hillier, D. J., Damineli, A., et al. 2009, ApJ, 698, 1698

Higgs, L. A., Feldman, P. A., \& Smolinski, J. 1978, ApJ, 220, L109

Jura, M., \& Kleinmann, S. G. 1990, ApJS, 73, 769

Kothes, R., \& Dougherty, S. M. 2007, A\&A, 468, 993

Lau, R. M., Herter, T. L., Morris, M. R., \& Adams, J. D. 2014, ApJ, 785, 120

Leitherer, C., \& Robert, C. 1991, ApJ, 377, 629 
Leitherer, C., Robert, C., \& Heckman, T. 1995, ApJS, 99, 173

Leitherer, C., Chapman, J. M., \& Koribalski, B. 1997, ApJ, 481, 898

Lobel, A., Israelian, G., de Jager, C., et al. 1998, A\&A, 330, 659

Lobel, A., Dupree, A. K., Stefanik, R. P., et al. 2003, ApJ, 583, 923

Mackey, J., Castro, N., Fossati, L., \& Langer, N. 2015, A\&A, 582, A24

McMullin, J. P., Waters, B., Schiebel, D., Young, W., \& Golap, K. 2007, in Astronomical Data Analysis Software and Systems XVI, eds. R. A. Shaw, F. Hill, \& D. J. Bell, ASP Conf. Ser., 376, 127

Morford, J. C., Fenech, D. M., Prinja, R. K., et al. 2017, A\&A, submitted

Muno, M., Law, C., Clark, J. S., et al. 2006, ApJ, 650, 203

Najarro, F., Figer, D. F., Hillier, D. J., Geballe, T. R., \& Kudritzki, R. P. 2009, ApJ, 691, 1816

Negueruela, I., Clark, J. S., \& Ritchie, B. W. 2010, A\&A, 516, A78

Oudmaijer, R. D., Groenewegen, M. A. T., Matthews, H. E., Blommaert, J. A. D. L., \& Sahu, K. C. 1996, MNRAS, 280, 1062

Peck, L. 2014, PhD Thesis, UCL

Peck, L. W., \& Fenech, D. M. 2013, Astron. Comput., 2, 54

Pittard, J. M. 2010, MNRAS, 403, 1633

Pittard, J., \& Dawson, B. 2018, MNRAS, 477, 5640

Prinja, R. K., \& Massa, D. L. 2013, A\&A, 559, A15

Prinja, R. K., Hodges, S. E., Urbaneja, M. A., \& Massa, D. L. 2010, MNRAS, 402, 641

Puls, J., Markova, N., Scuderi, S., et al. 2006, A\&A, 454, 625
Ritchie, B. W., Clark, J. S., Negueruela, I., \& Najarro, F. 2009, A\&A, 507, 1597 Sana, H., de Mink, S. E., de Koter, A., et al. 2012, Science, 337, 444 Sana, H., de Koter, A., de Mink, S. E., et al. 2013, A\&A, 550, A107 Sault, B., \& Killeen, N. 2004, Miriad (Multichannel Image Reconstruction, Image Analysis and Display) Users Guide, 19, https://www.cfa. harvard.edu/sma/miriad/manuals/ATNFuserguide_US.pdf

Sault, R. J., Teuben, P. J., \& Wright, M. C. H. 1995, in Astronomical Data Analysis Software and Systems IV, eds. R. A. Shaw, H. E. Payne, \& J. J. E. Hayes, ASP Conf. Ser., 77, 433

Shenoy, D., Humphreys, R., Jones, T., et al. 2016, AJ, 151, 51

Stevens, I. R., \& Hartwell, J. M. 2003, MNRAS, 339, 280

Stevens, I. R., Blondin, J. M., \& Pollock, A. M. T. 1992, ApJ, 386, 265

Stickland, D. J., \& Harmer, D. L. 1978, A\&A, 70, L53

Sundqvist, J. O., Puls, J., Feldmeier, A., \& Owocki, S. P. 2011, A\&A, 528, A64 Surlan, B., Hamann, W.-R., Kubát, J., Oskinova, L. M., \& Feldmeier, A. 2012, A\&A, 541, A37

Sylvester, R. J., Skinner, C. J., \& Barlow, M. J. 1998, MNRAS, 301, 1083

Westerlund, B. 1961, PASP, 73, 51

Wright, A. E., \& Barlow, M. J. 1975, MNRAS, 170, 41

Yusef-Zadeh, F., \& Morris, M. 1991, ApJ, 371, L59

Yusef-Zadeh, F., Morris, M., \& van Gorkom, J. H. 1989, in The Center of the Galaxy, ed. M. Morris, IAU Symp., 136, 275

Yusef-Zadeh, F., Nord, M., Wardle, M., et al. 2003, ApJ, 590, L103 


\section{Appendix A: Tables}

Table A.1. Flux densities for known stellar sources from FULL9 dataset.

\begin{tabular}{|c|c|c|c|}
\hline Source & Flux $_{\text {Full9 }}$ & Flux $_{\text {Do10 }}$ & Notes \\
\hline \multirow[t]{2}{*}{ WR A } & $0.48 \pm 0.04$ & $0.5 \pm 0.06$ & \\
\hline & $1.34 \pm 0.09$ & - & \\
\hline WR D & $0.34 \pm 0.06$ & - & \\
\hline \multirow{2}{*}{ WR B } & $3.24 \pm 0.06^{c}$ & - & Crowded \\
\hline & $9.50 \pm 0.18^{t}$ & $4.3 \pm 0.4^{r}$ & \\
\hline WR I & $0.45 \pm 0.03$ & - & Isolated \\
\hline WR V & - & $0.4 \pm 0.06$ & Crowded \\
\hline WR L & $0.40 \pm 0.04$ & $0.4 \pm 0.06$ & Isolated \\
\hline WR S & $0.08 \pm 0.03$ & $0.3 \pm 0.06$ & \\
\hline WR E & $0.11 \pm 0.02$ & - & Isolated \\
\hline WR F & $0.34 \pm 0.03$ & $0.3 \pm 0.06$ & Isolated \\
\hline WR M & $0.16 \pm 0.04$ & - & \\
\hline W16a & $1.79 \pm 0.09$ & $1.6 \pm 0.3$ & Crowded \\
\hline \multirow[t]{2}{*}{$\mathrm{W} 12 \mathrm{a}$} & $1.66 \pm 0.04^{c}$ & - & \\
\hline & $3.28 \pm 0.08$ & $2.9 \pm 0.3^{r}$ & \\
\hline \multirow[t]{2}{*}{ W4a } & $1.79 \pm 0.04^{c}$ & $0.8 \pm 0.08$ & \\
\hline & $4.19 \pm 0.11^{t}$ & $2.2 \pm 0.2^{r}$ & \\
\hline W32 & $0.16 \pm 0.04$ & $0.4 \pm 0.06$ & Crowded \\
\hline \multirow[t]{2}{*}{ W265 } & $0.94 \pm 0.06^{c}$ & - & Isolated \\
\hline & $2.72 \pm 0.16^{t}$ & $2.3 \pm 0.3^{r}$ & \\
\hline \multirow[t]{2}{*}{ W237 } & $1.26 \pm 0.04^{c}$ & $1.8 \pm 0.2$ & Isolated \\
\hline & $7.01 \pm 0.22^{t}$ & $5.6 \pm 2.2^{r}$ & \\
\hline W75 & $0.26 \pm 0.04$ & $0.3 \pm 0.06$ & Isolated \\
\hline \multirow[t]{2}{*}{ W20 } & $2.39 \pm 0.05^{c}$ & - & \\
\hline & $16.62 \pm 0.27^{t}$ & $3.8 \pm 0.4^{r}$ & \\
\hline W26 & $152.15 \pm 0.33$ & $20.1 \pm 2.0^{r}$ & \\
\hline \multirow[t]{2}{*}{ W17 } & $0.98 \pm 0.05$ & - & Crowded \\
\hline & $1.27 \pm 0.07$ & $1.7 \pm 0.2$ & \\
\hline W243 & $1.65 \pm 0.05$ & $1.5 \pm 0.2$ & Isolated \\
\hline \multirow[t]{2}{*}{ W9 } & $30.47 \pm 0.09^{c}$ & $24.9 \pm 2.5$ & Crowded \\
\hline & $80.8 \pm 0.5^{t}$ & $30.5 \pm 3.0^{r}$ & \\
\hline \multirow{2}{*}{ D09-R1 } & $1.20 \pm 0.04^{c}$ & $0.7 \pm 0.07$ & Crowded \\
\hline & $4.66 \pm 0.14^{t}$ & $6.5 \pm 1.2^{r}$ & \\
\hline D09-R2 & $0.88 \pm 0.08$ & $0.7 \pm 0.06$ & Crowded \\
\hline W15 & $0.84 \pm 0.10$ & $0.6 \pm 0.06$ & \\
\hline
\end{tabular}

Notes. Fluxes are taken from FULL9 dataset, measured with SEAC, assuming $\sigma_{\mathrm{f}}=3, \sigma_{\mathrm{s}}=5$, with consideration of segmentation where necessary. Flux densities are given in mJy. Core and total flux densities are given for relevant sources, noted by $c, t$ respectively. Flux density values are compared to fluxes found in $\mathrm{D} 10$, at $8.6 \mathrm{GHz}$, where the superscript $r$ refers to objects that were found to be spatially resolved. 
Table A.2. Flux values of source detections from FuLLCONCAT, for ALMA detected sources (F18).

\begin{tabular}{|c|c|c|c|}
\hline FCP18 Source & RA & Dec & Flux $_{\text {FULLCONCAT }}(\mathrm{mJy})$ \\
\hline 2 & 164658.60265 & -455031.4920 & $1.09 \pm 0.02$ \\
\hline 3 & 164658.88981 & -455028.7929 & $\begin{array}{l}1.88 \pm 0.02^{c} \\
3.92+0.03^{t}\end{array}$ \\
\hline 6 & 164659.40638 & -455037.1942 & $\begin{array}{l}2.11 \pm 0.02^{c} \\
4.88 \pm 0.04^{t}\end{array}$ \\
\hline 8 & 164659.57887 & -455026.6947 & $0.31 \pm 0.04$ \\
\hline 12 & 16471.01416 & -455036.2975 & $0.75 \pm 0.04$ \\
\hline 16 & 16471.81753 & -455123.0987 & $2.11 \pm 0.04$ \\
\hline 22 & 16472.33444 & -455121.5992 & $\begin{array}{l}0.27 \pm 0.02^{c} \\
0.66 \pm 0.04^{t}\end{array}$ \\
\hline 27 & 16472.85135 & -455119.1996 & $1.12 \pm 0.05$ \\
\hline 35 & 16473.68413 & -455116.5000 & $0.31 \pm 0.04$ \\
\hline 37 & 16473.74162 & -455024.0000 & $0.38 \pm 0.06$ \\
\hline 39 & 16473.88514 & -45516.3000 & $\begin{array}{l}1.80 \pm 0.03^{c} \\
4.63 \pm 0.09^{t}\end{array}$ \\
\hline 49 & 16474.22972 & -455116.2000 & $0.35 \pm 0.05$ \\
\hline 60 & 16475.06241 & -45512.3997 & $1.59 \pm 0.02$ \\
\hline 64 & 16475.75158 & -45517.1991 & $2.81 \pm 0.03$ \\
\hline 72 & 16476.29690 & -455044.9985 & $0.60 \pm 0.03$ \\
\hline 73 & 16476.38337 & -455113.7984 & $1.56 \pm 0.02$ \\
\hline 77 & 16476.46965 & -455123.9983 & $0.33 \pm 0.03$ \\
\hline 80 & 16476.81406 & -455110.4978 & $1.24 \pm 0.05$ \\
\hline 82 & 16477.30222 & -455111.0970 & $0.45 \pm 0.04$ \\
\hline 85 & 16477.87660 & -455115.2959 & $0.09 \pm 0.02$ \\
\hline 86 & 16478.30665 & -455043.4949 & $0.32 \pm 0.03$ \\
\hline 87 & 16478.33615 & -455119.7949 & $0.40 \pm 0.03$ \\
\hline 90 & 16478.50825 & -455111.0944 & $2.13 \pm 0.05$ \\
\hline 91 & 16478.53623 & -455038.6944 & $0.30 \pm 0.05$ \\
\hline 93 & 16478.70934 & -455114.6939 & $2.28 \pm 0.04$ \\
\hline 95 & 16478.87997 & -45506.2935 & $0.48 \pm 0.02$ \\
\hline 97 & 16478.93797 & -455030.2933 & $0.28 \pm 0.03$ \\
\hline 99 & 16479.16849 & -45513.2927 & $0.28 \pm 0.03$ \\
\hline 100 & 16479.36973 & -455112.2921 & $0.76 \pm 0.04$ \\
\hline 101 & 164710.77535 & -455030.2875 & $0.35 \pm 0.04$ \\
\hline
\end{tabular}

Notes. The source determinations are gathered from SEAC, with the use of the segmentation tools when necessary. Thresholds were set at $\sigma_{\mathrm{f}}=3$, $\sigma_{\mathrm{s}}=5$. For any significantly extended sources, core and total regions are specified with the use of superscripts $c$ and $t$ respectively.

Table A.3. Spectral index values found for ALMA detected sources (F18).

\begin{tabular}{lllll}
\hline \hline FCP18 Source & Flux $_{\text {TAPER } 5}(\mathrm{mJy})$ & Flux $_{\text {TAPER } 9}(\mathrm{mJy})$ & Flux $_{\text {TAPERALMA }}(\mathrm{mJy})$ & Spectral index $(\alpha)$ \\
\hline 3 & $2.69 \pm 0.04$ & $1.91 \pm 0.06$ & $1.18 \pm 0.14$ & $-0.26 \pm 0.05$ \\
6 & $3.98 \pm 0.05$ & $3.75 \pm 0.08$ & $1.04 \pm 0.12$ & $-0.49 \pm 0.05$ \\
16 & $1.66 \pm 0.07$ & $1.91 \pm 0.07$ & $0.96 \pm 0.06$ & $-0.23 \pm 0.03$ \\
22 & - & $0.30 \pm 0.05$ & $0.10 \pm 0.03$ & $-0.81 \pm 0.12$ \\
27 & $0.54 \pm 0.09$ & $0.85 \pm 0.06$ & $0.34 \pm 0.05$ & $-0.27 \pm 0.07$ \\
39 & $2.04 \pm 0.08$ & $2.28 \pm 0.07$ & $1.57 \pm 0.15$ & $-0.10 \pm 0.04$ \\
60 & $1.42 \pm 0.07$ & $1.05 \pm 0.06$ & $0.68 \pm 0.12$ & $-0.23 \pm 0.07$ \\
64 & $2.33 \pm 0.08$ & $1.89 \pm 0.07$ & $1.67 \pm 0.17$ & $-0.09 \pm 0.04$ \\
72 & $0.40 \pm 0.08$ & $0.49 \pm 0.09$ & $0.26 \pm 0.07$ & $-0.19 \pm 0.13$ \\
73 & $1.20 \pm 0.05$ & $1.26 \pm 0.06$ & $0.72 \pm 0.12$ & $-0.19 \pm 0.07$ \\
82 & - & $0.34 \pm 0.05$ & $0.16 \pm 0.05$ & $-0.82 \pm 0.13$ \\
90 & $2.00 \pm 0.15$ & $1.81 \pm 0.09$ & $0.74 \pm 0.12$ & $-0.35 \pm 0.07$ \\
93 & $2.00 \pm 0.14$ & $2.28 \pm 0.10$ & $0.82 \pm 0.13$ & $-0.34 \pm 0.07$ \\
95 & $0.57 \pm 0.06$ & $0.62 \pm 0.06$ & $0.33 \pm 0.06$ & $-0.21 \pm 0.08$ \\
99 & - & $0.25 \pm 0.07$ & $0.11 \pm 0.04$ & $-0.50 \pm 0.16$ \\
\hline
\end{tabular}

Notes. Flux values were measured from TAPER5, TAPER 9 and TAPERALMA. All data are tapered to contain the same range of $u-v$ visibilities. When possible, core components of the sources are used in order for better source comparison between the differently resolved radio and millimetre observations. 
Table A.4. Flux densities of radio sources from FullCONCAT dataset with no known counterparts.

\begin{tabular}{|c|c|c|c|}
\hline HA19 Source ID & RA & Dec & $\operatorname{Flux}_{\mathrm{ATCA}}(\mathrm{mJy})$ \\
\hline 3 & 164655.96080 & -455046.1824 & $0.12 \pm 0.03$ \\
\hline 4 & 164656.10430 & -455047.6830 & $0.05 \pm 0.01$ \\
\hline 5 & 164656.22091 & -45502.3835 & $0.27 \pm 0.04$ \\
\hline 6 & 164656.47741 & -455051.5846 & $0.31 \pm 0.03$ \\
\hline 8 & 164656.88056 & -455018.2862 & $0.08 \pm 0.02$ \\
\hline 10 & 164657.16625 & -455058.7872 & $0.06 \pm 0.02$ \\
\hline 11 & 164657.59801 & -455025.7888 & $0.25 \pm 0.04$ \\
\hline 12 & 164659.20651 & -454950.9937 & $0.21 \pm 0.03$ \\
\hline 14 & 164659.52162 & -455019.1945 & $0.09 \pm 0.03$ \\
\hline 16 & 16470.38207 & -45512.3964 & $0.25 \pm 0.04$ \\
\hline 17 & 16470.66916 & -45515.3970 & $0.06 \pm 0.02$ \\
\hline 23 & 16471.61678 & -45 510.5984 & $0.10 \pm 0.03$ \\
\hline 31 & 16473.10981 & -455117.3998 & $0.12 \pm 0.03$ \\
\hline 32 & 16473.25367 & -454959.9998 & $1.21 \pm 0.07$ \\
\hline 33 & 16473.42563 & -455136.5999 & $0.13 \pm 0.02$ \\
\hline 43 & 16475.52210 & -455137.7993 & $0.38 \pm 0.04$ \\
\hline 44 & 16475.69449 & -455147.0992 & $0.07 \pm 0.02$ \\
\hline 48 & 16476.64016 & -45498.6981 & $0.11 \pm 0.03$ \\
\hline 50 & 16477.04369 & -45514.7975 & $0.32 \pm 0.04$ \\
\hline 51 & 16477.27263 & -455017.6971 & $0.12 \pm 0.02$ \\
\hline 54 & 16477.67579 & -455125.7963 & $0.32 \pm 0.03$ \\
\hline 55 & 16477.76142 & -455058.4961 & $0.10 \pm 0.03$ \\
\hline 56 & 16477.87526 & -45505.9959 & $0.49 \pm 0.04$ \\
\hline 57 & 16477.90541 & -455119.7958 & $0.67 \pm 0.05$ \\
\hline 58 & 16477.93390 & -45518.3958 & $0.29 \pm 0.04$ \\
\hline 59 & 16477.99108 & -455056.0956 & $0.35 \pm 0.04$ \\
\hline 60 & 16478.07573 & -454942.8954 & $0.10 \pm 0.02$ \\
\hline 61 & 16478.07736 & -45512.9954 & $0.55 \pm 0.04$ \\
\hline 62 & 16478.33501 & -455027.2949 & $0.61 \pm 0.08$ \\
\hline 65 & 16478.70722 & -454944.3939 & $0.09 \pm 0.02$ \\
\hline 67 & 16478.90891 & -455016.1934 & $0.07 \pm 0.02$ \\
\hline 69 & 16479.05354 & -455059.3930 & $0.07 \pm 0.02$ \\
\hline 70 & 16479.08041 & -454946.7929 & $0.19 \pm 0.03$ \\
\hline 72 & 16479.34005 & -455035.9922 & $0.94 \pm 0.05$ \\
\hline 73 & 16479.56953 & -455028.7915 & $0.63 \pm 0.04$ \\
\hline 74 & 16479.68496 & -455049.7912 & $0.39 \pm 0.03$ \\
\hline 76 & 164710.17335 & -455059.0896 & $0.21 \pm 0.03$ \\
\hline 77 & 164710.28717 & -455026.0892 & $0.47 \pm 0.04$ \\
\hline 78 & 164710.43165 & -455055.4887 & $1.63 \pm 0.06$ \\
\hline 79 & 164710.46117 & -455120.3886 & $0.19 \pm 0.03$ \\
\hline 81 & 164710.83417 & -455111.3872 & $0.08 \pm 0.02$ \\
\hline 82 & 164710.94981 & -455133.8868 & $1.20 \pm 0.06$ \\
\hline 83 & 164711.17729 & -455030.5859 & $0.55 \pm 0.05$ \\
\hline 85 & 164711.34994 & -455041.3853 & $0.05 \pm 0.01$ \\
\hline 86 & 164711.52155 & -455023.9846 & $0.32 \pm 0.04$ \\
\hline 87 & 164711.55173 & -45512.9844 & $0.14 \pm 0.03$ \\
\hline 88 & 164711.75329 & -455117.3836 & $0.14 \pm 0.02$ \\
\hline 89 & 164711.78273 & -455135.9835 & $0.14 \pm 0.02$ \\
\hline 90 & 164711.86639 & -455032.6831 & $0.11 \pm 0.02$ \\
\hline 91 & 164712.03810 & -455018.8824 & $0.36 \pm 0.04$ \\
\hline 92 & 164712.09525 & -455012.2821 & $0.84 \pm 0.07$ \\
\hline 93 & 164712.38490 & -455114.0808 & $0.14 \pm 0.03$ \\
\hline 94 & 164714.33810 & -455124.8708 & $0.24 \pm 0.04$ \\
\hline
\end{tabular}

Notes. These fluxes have been found from a run-through of the SEAC algorithm, without the use of the segmentation tool, with thresholds of $\sigma_{\mathrm{f}}=3$ and $\sigma_{\mathrm{s}}=5$. Source numbers are assigned to the definitive radio catalogue output of 94 radio sources detected in FULLCONCAT, ordered by increasing RA. 


\section{Appendix B: Figures}

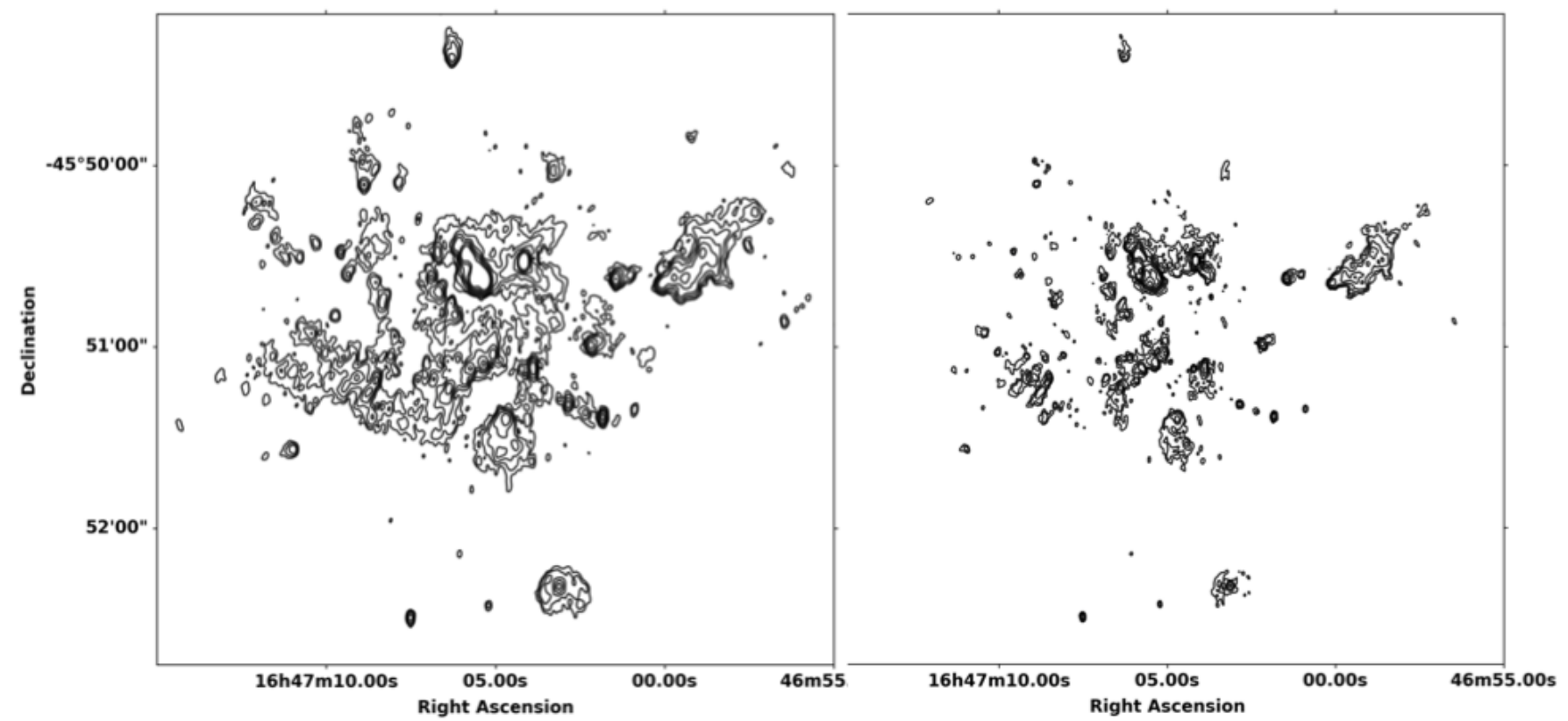

Fig. B.1. ATCA contours from FULL5 dataset (left), and FULL9 dataset (right), using non-primary beam corrected images. Contour levels are set at $-3,3,6,9,12,24,28$ and $192 \times \sigma$, where $\sigma$ is the image rms, set at 0.03 mJy for FULL5 and 0.04 mJy for FULL9.

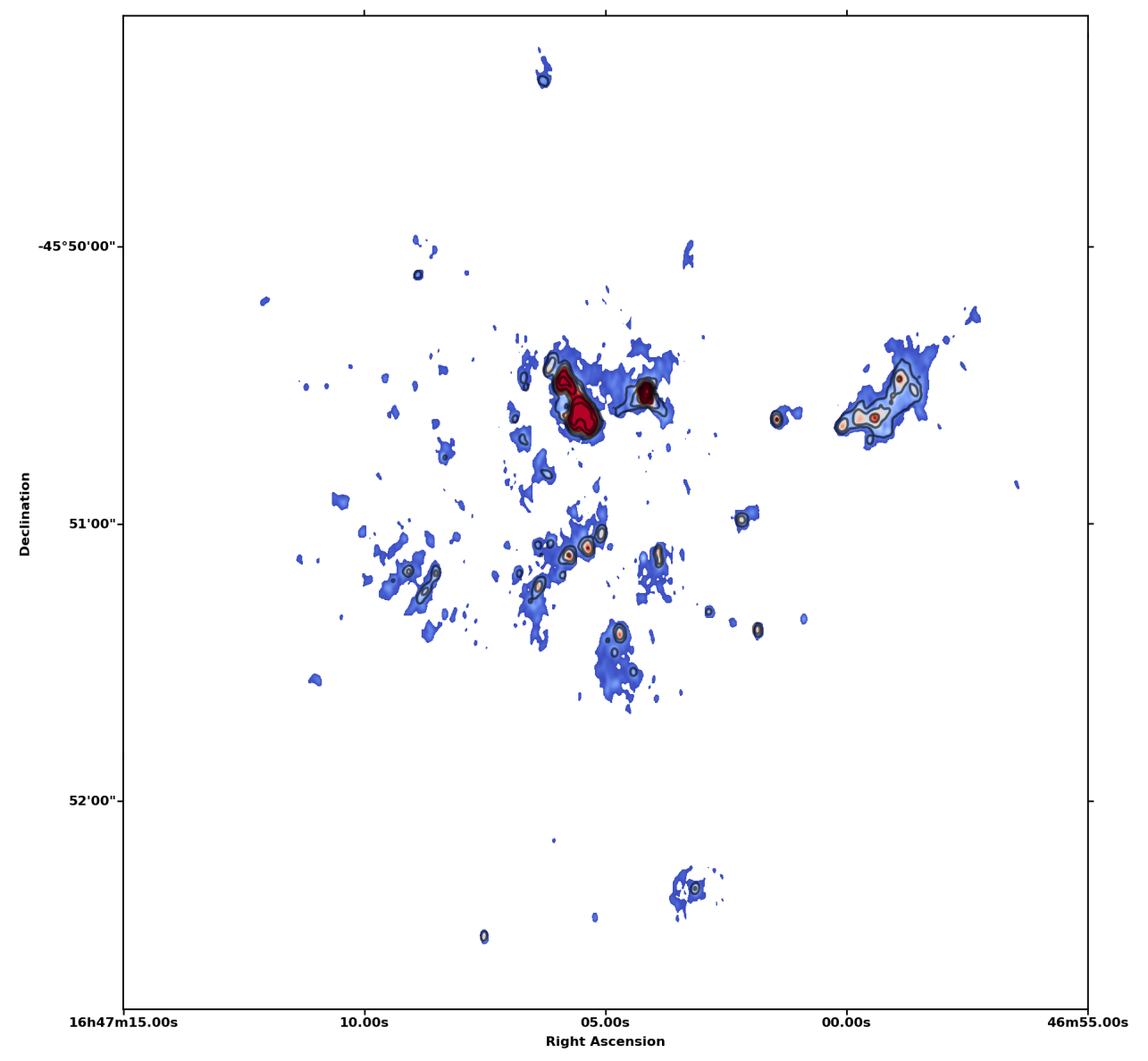

Fig. B.2. ATCA colour-scale of FULL9 dataset (using non-primary beam corrected image). Contours are overlaid from the $8.6 \mathrm{GHz}$ D10 observations. The colourscale ranges from 0.12 to $2.0 \mathrm{mJy}_{\text {beam }}^{-1}$, and contour levels are set at $-3,3,6,9$, $12,24,48,96,192 \times \sigma$, where $\sigma$ is set at $0.6 \mathrm{mJy}^{\text {beam }}{ }^{-1}$, as used in D10. 

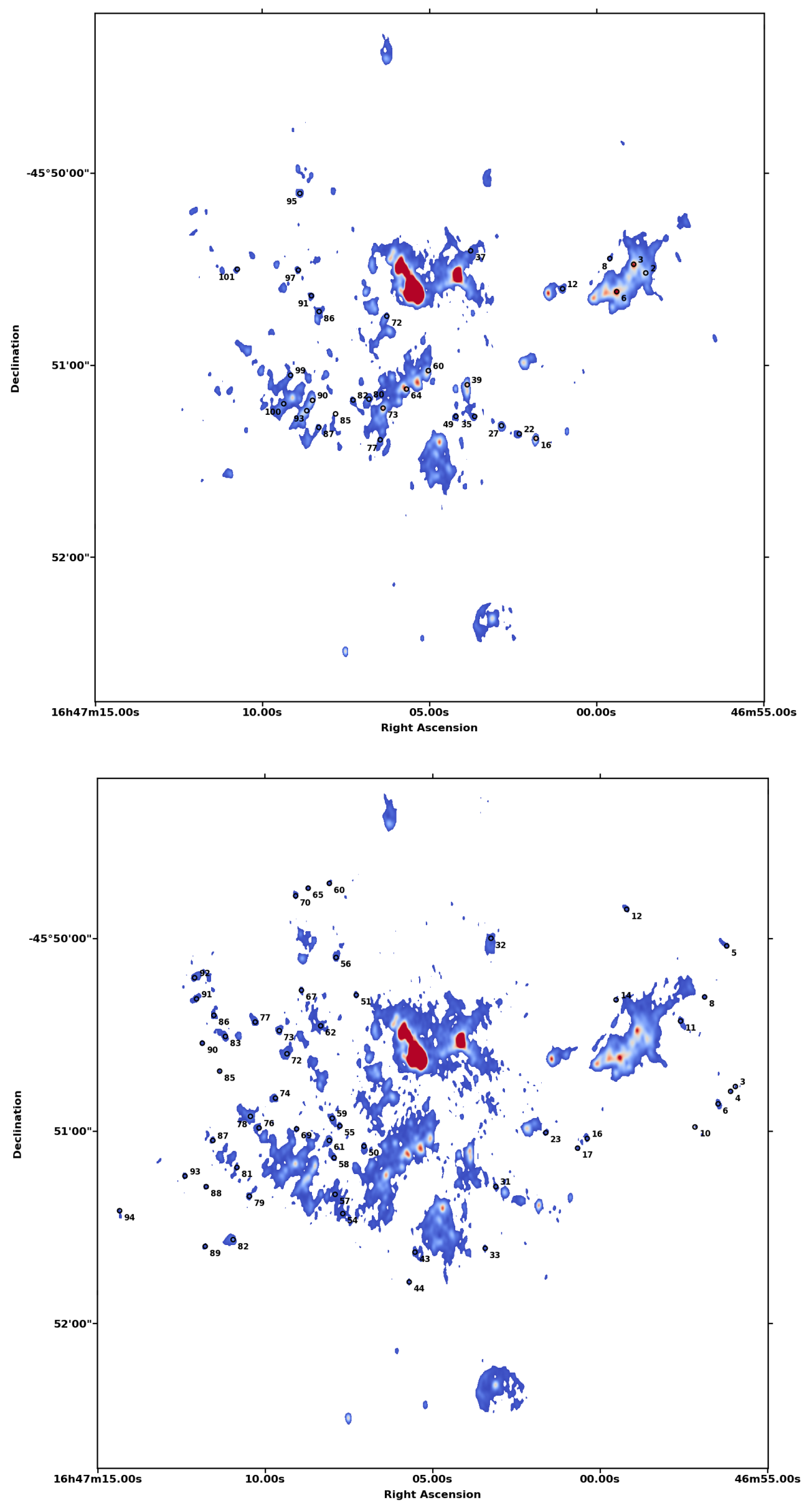

Fig. B.3. ATCA colour-scale (from non-primary beam corrected image) of FULLCONCAT dataset. The colour-scale is set from 0.1 to $2.0 \mathrm{mJy}^{\text {beam }}{ }^{-1}$. Unknown sources identified in the ALMA observations (F18) were compared to SEAC detections from the ATCA dataset and sources that were detected in both are indicated with circles and labelled with the source number as given in F18. Positions and fluxes are listed in Table A.2.
Fig. B.4. ATCA colour-scale (from nonprimary beam corrected image) of FULLCONCAT dataset. The colour-scale is set from 0.1 to $2.0 \mathrm{mJy}$ beam $^{-1}$. Unknown sources with no known counterparts are indicated with circles, and labelled. Positions and fluxes are listed in Table A.4. 


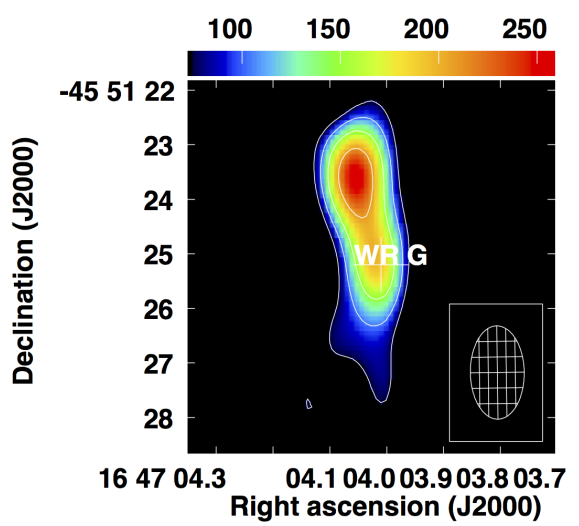

(a)

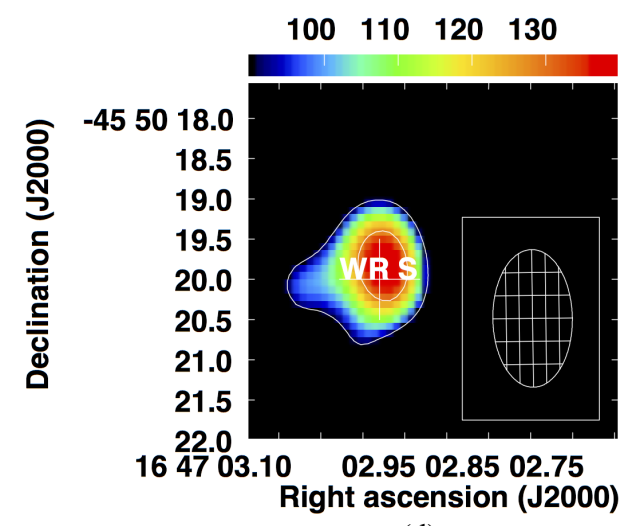

(d)

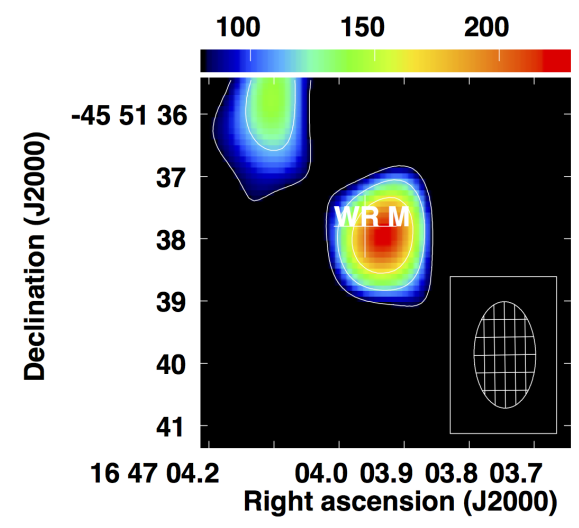

(g)

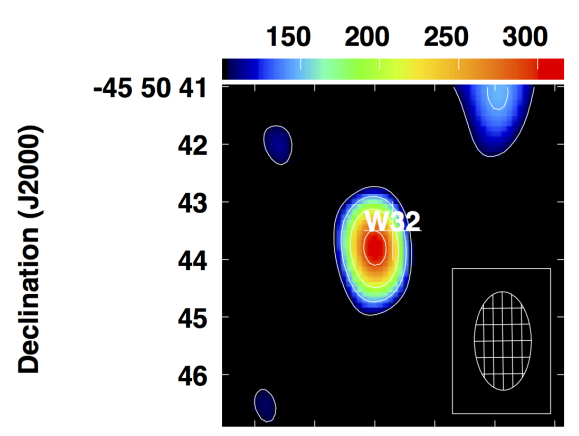

$164703.9 \quad 03.703 .603 .503 .4$ Right ascension (J2000)

(j)

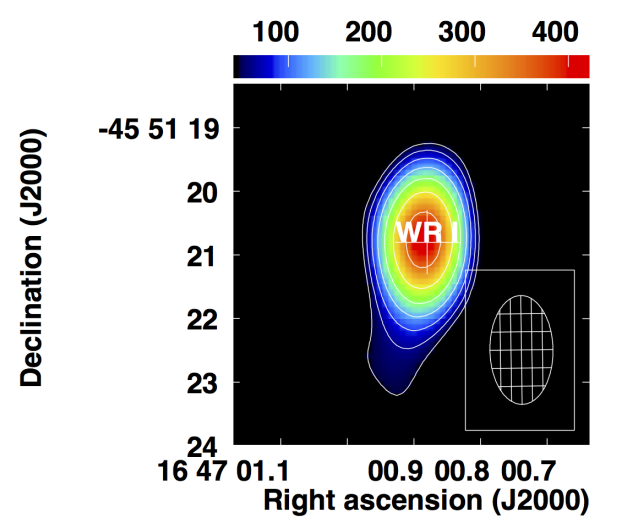

(b)

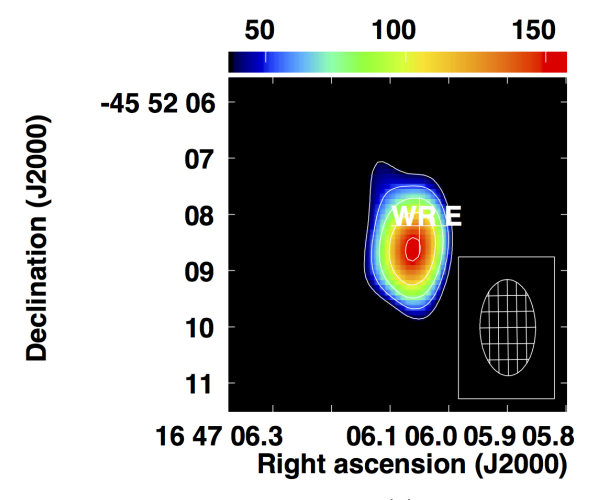

(e)

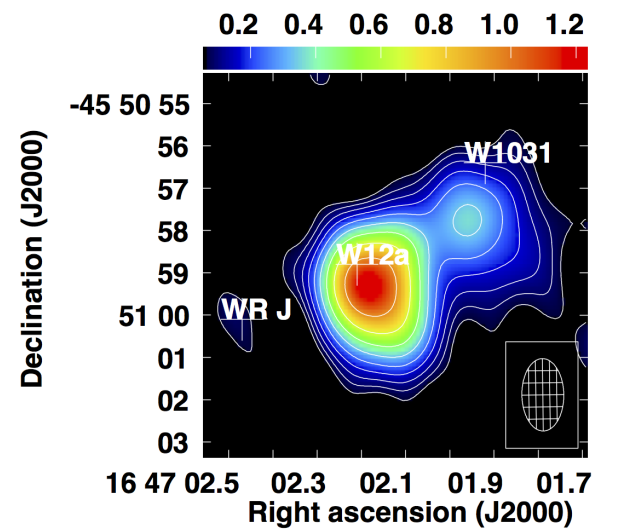

(h)

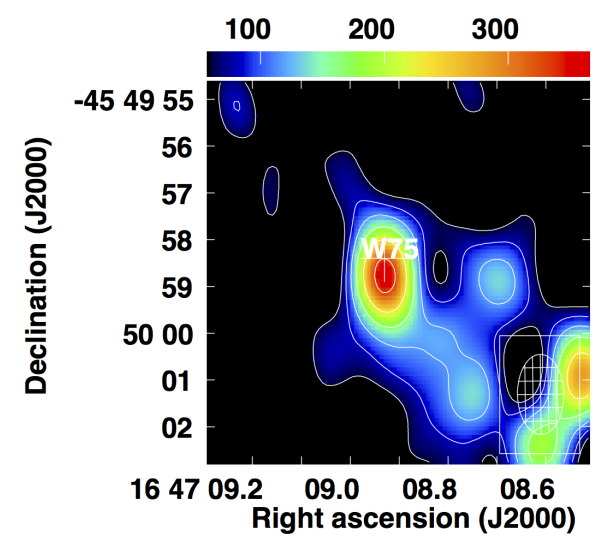

(k)

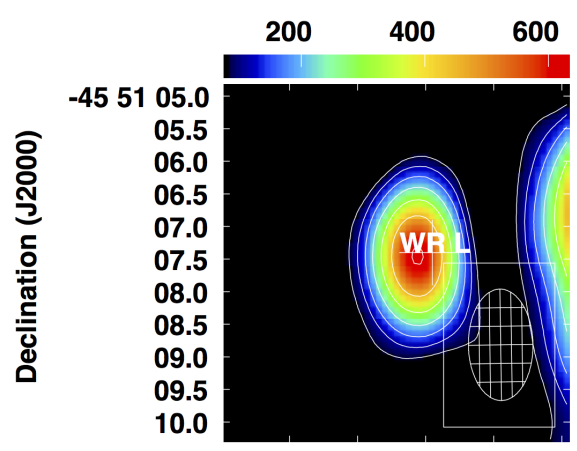

164704.504 .404 .304 .204 .104 .0 Right ascension (J2000)

(c)

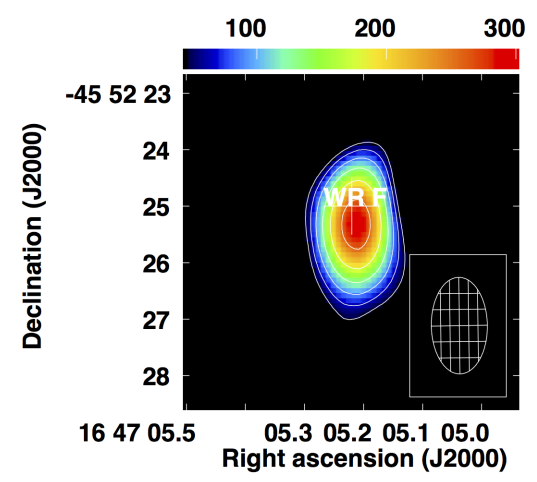

(f)

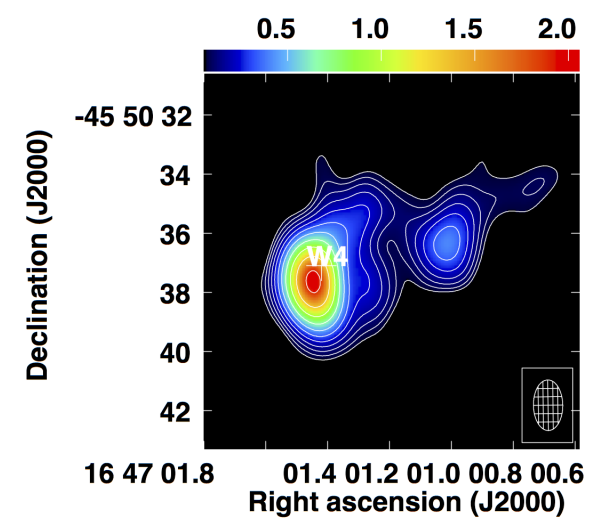

(i)

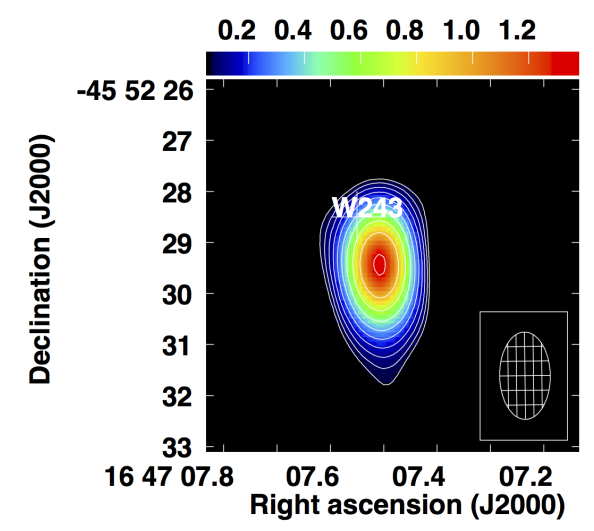

(1)

Fig. B.5. ATCA colour-scale images from FULLCONCAT dataset of detected stellar sources, using non-primary beam corrected images. Contours are plotted at $1,1.4,2,2.8,4,5.7,8,11.3,16,22.6,32,45.25,64,90.50 \times \sigma .(a)$ WR G. $(b)$ WR I. $(c)$ WR L. $(d)$ WR S. $(e)$ WR E. $(f)$ WR F. $(g)$ WR M. (h) W12a. (i) W4a. (j) W32. (k) W75. (l) W243. (m) W20. $(n)$ W26. $(o)$ W17. (p) D09-R1. (q) D09-R2. $(r)$ W15. $(s)$ W10. $(t)$ W18. $(u)$ W19. (v) W1031. (w) W1056. 


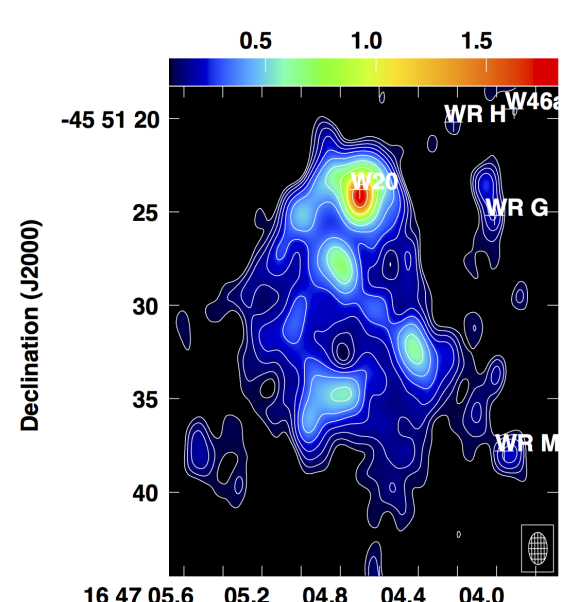

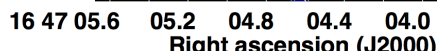

(m)

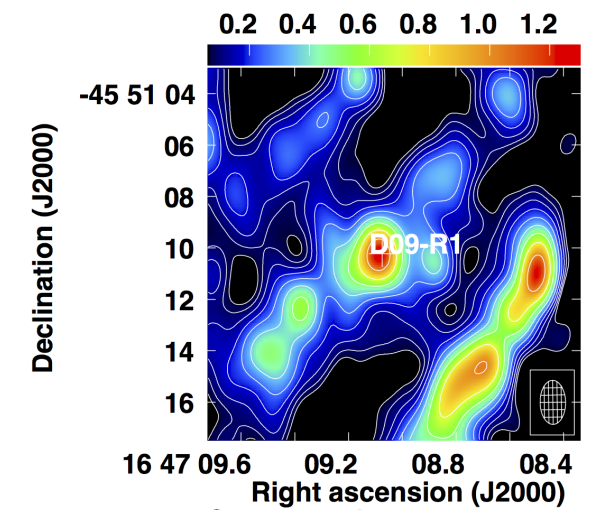

(p)

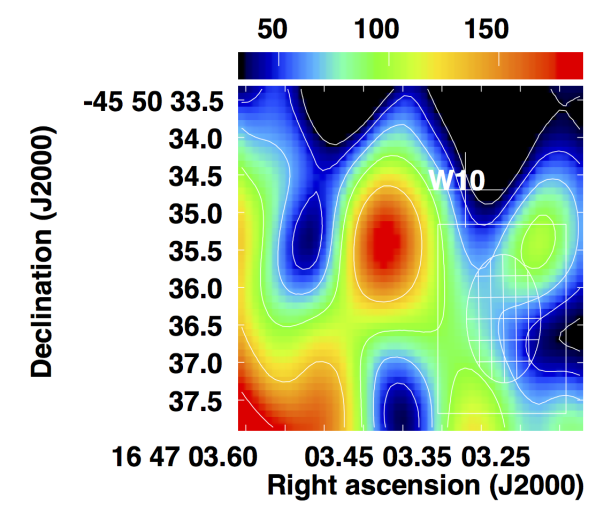

(s)

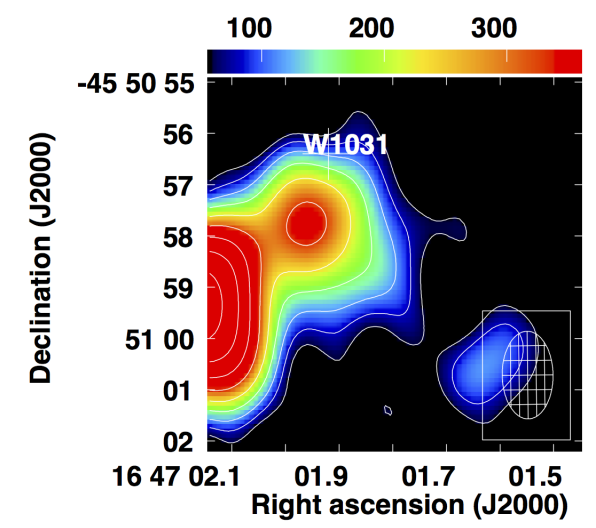

(v)

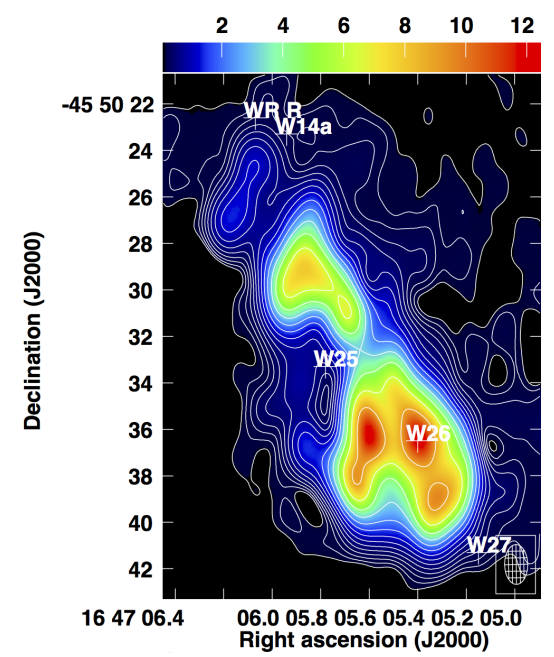

(n)

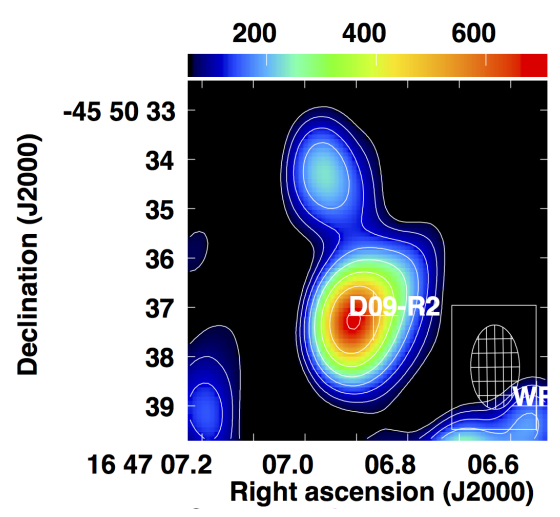

(q)

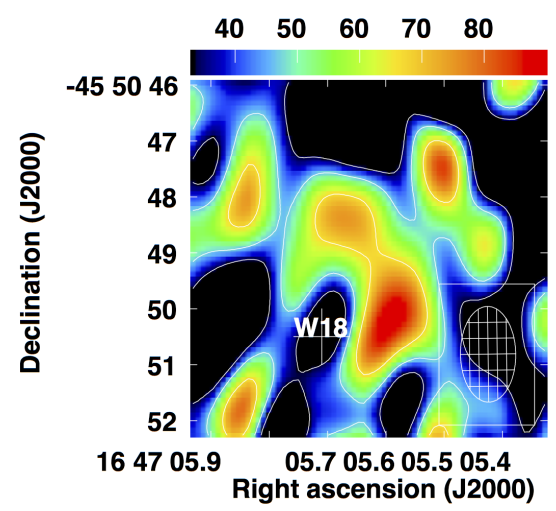

(t)

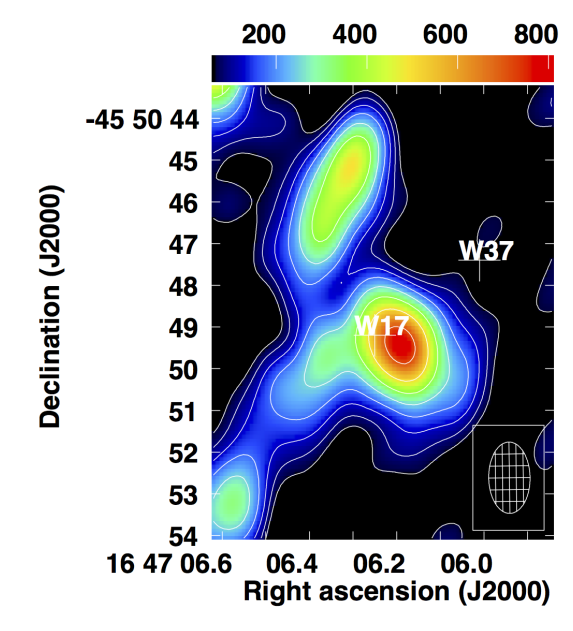

(o)

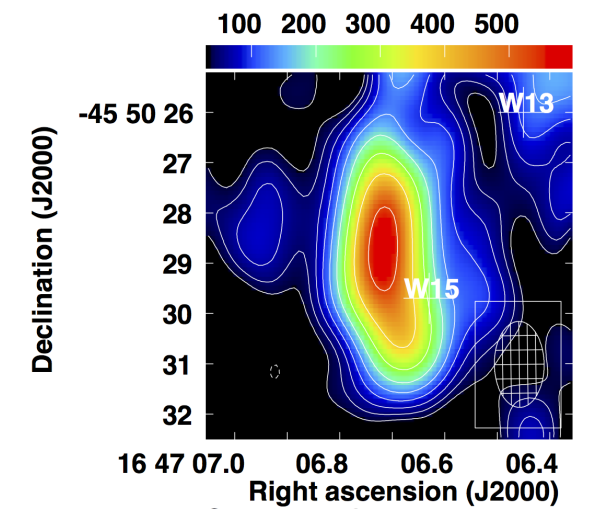

(r)

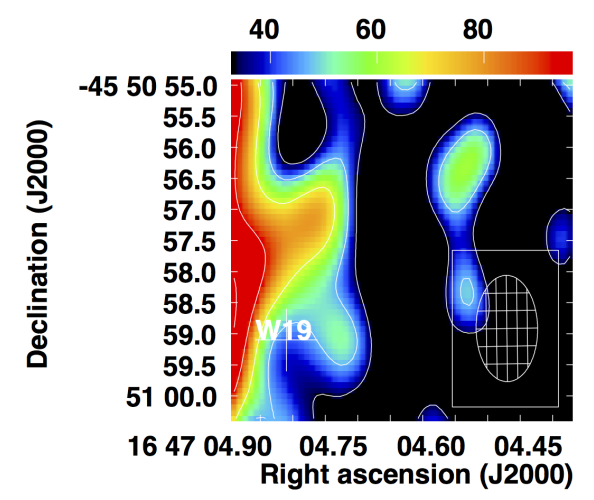

(u)

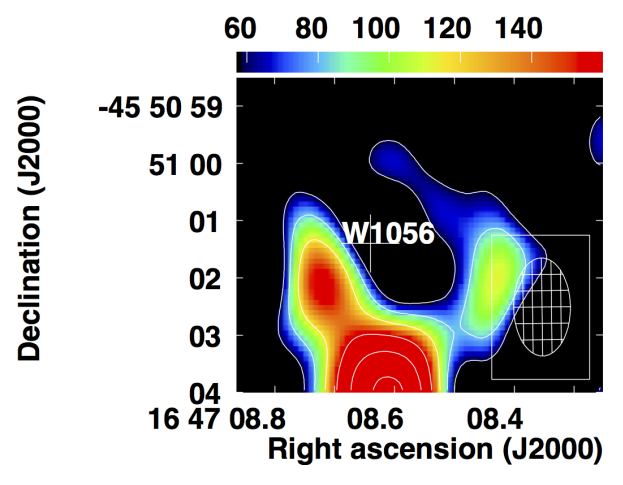

(w)

Fig. B.5. continued. 